\title{
Knowledge partitioning in categorization
}

\author{
Lee-Xieng Yang
}

This thesis is presented for the degree of

Doctor of Philosophy

Of The University of Western Australia

School of Psychology

2003 


\section{Acknowledgements}

Thanks to Prof. Stephan Lewandowsky, my supervisor, for introducing me to

cognitive modeling, inspiring me to link different research fields and to work in a logical manner, and for encouraging me to improve my English. Thanks to Simon Farrell, Mike

Kalish, John Kruschke, Mike Erickson and many others for their useful discussions.

Thanks to Joe Clare, Leo Roberts, Sato Jupiter and Simon Farrell for their help to my

writing. Thanks to friends and family for making it a bearable four years. Thankyou to

lovely Weinn for making it fun not to do my thesis. And thanks to any body I've missed. 


\section{Source of chapters}

This thesis is completely and utterly my own work. However, several of the

chapters are based on published articles co-authored with Stephan Lewandowsky. In all cases, simulations and chapters were independently accomplished.

Chapter 2, Chapter 3 and part of Chapter 7 are based on Yang L-X, and Lewandowsky, S. (2003). Context-gated knowledge partitioning in categorization.

Journal of Experimental Psychology: Learning, Memory and Cognition, 29, 663-679.

Chapter 4, Chapter 6, and part of Chapter 7 are based on Yang L-X, and Lewandowsky, S. (2003). Knowledge partitioning in categorization: Constrains on exemplar models. Manuscript submitted for publication. 
Table of contents

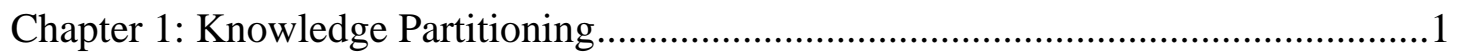

Expert Knowledge and Knowledge Partitioning............................................1

Laboratory Evidence of Knowledge Partitioning: Function Learning............3

Knowledge Partitioning is a Phenomenon As Well As a Theory ...................6

Chapter 2: Conceptual Category Learning (I).......................................................

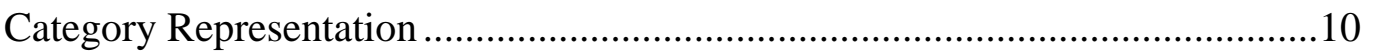

Heterogeneous Category Representations …....................................................12

Partitioning Knowledge in Categorization.........................................................15

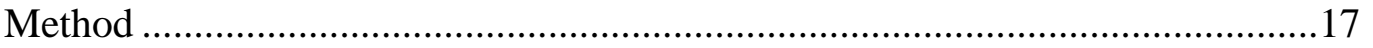

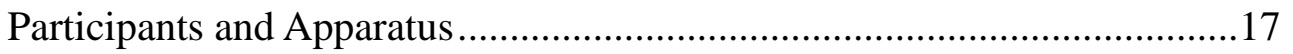

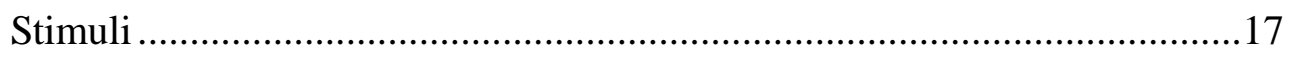

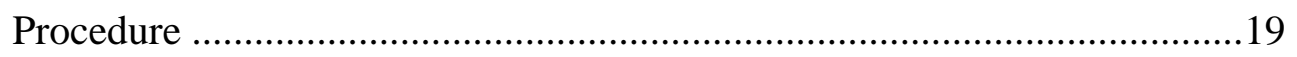

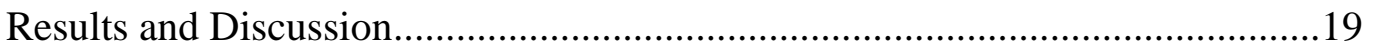

Training Performance...........................................................................19

Transfer Performance ............................................................................20

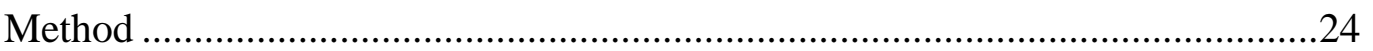

Participants ......................................................................................24

Stimuli and Procedure ….......................................................................24

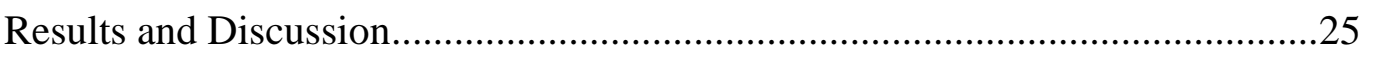

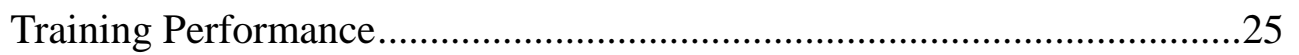

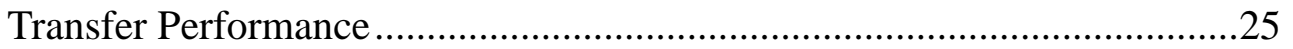

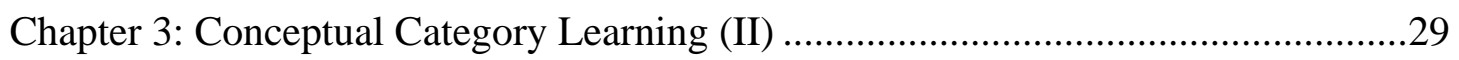

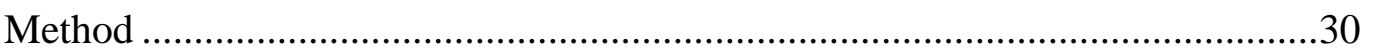

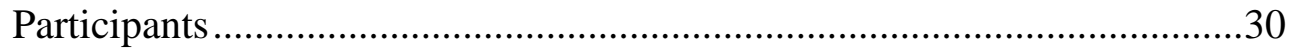

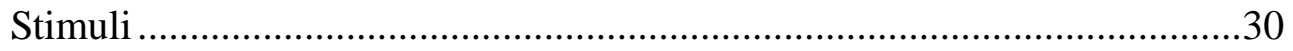

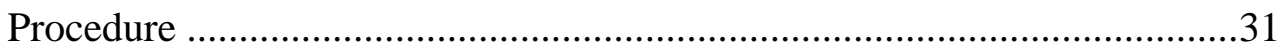

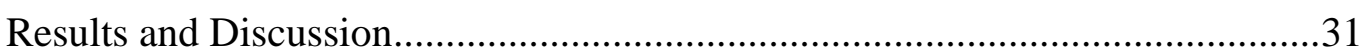

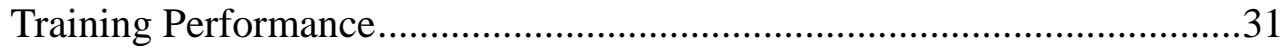

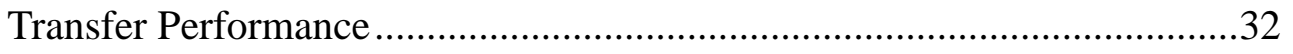

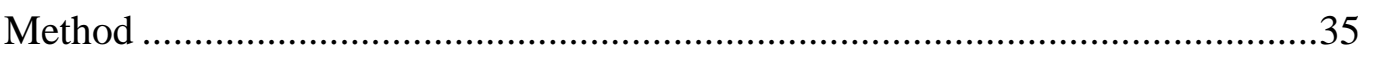

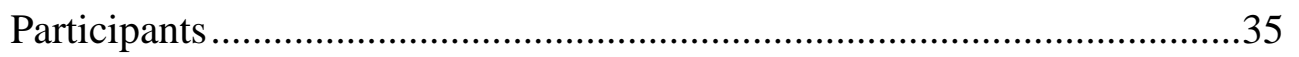

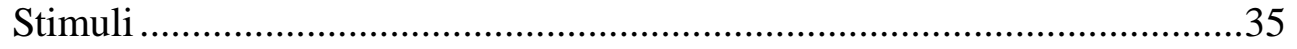

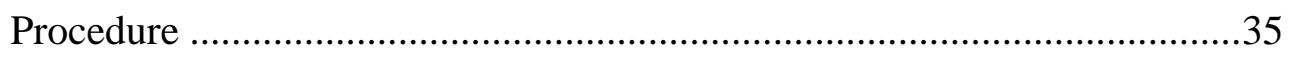

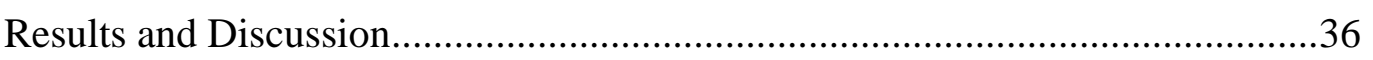

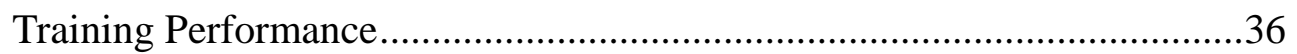

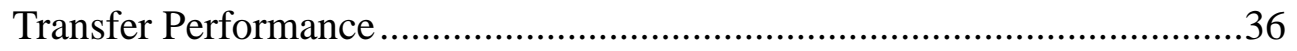

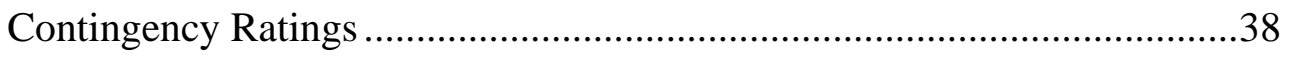


Limitations of the First Four Experiments .................................................39

Chapter 4: Perceptual Category Learning ............................................................40

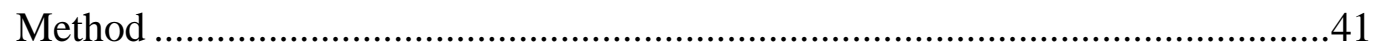

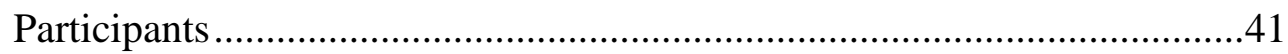

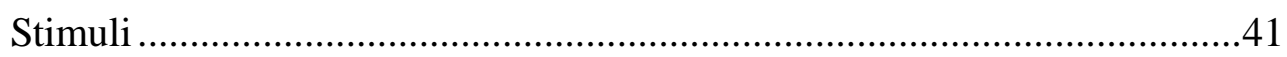

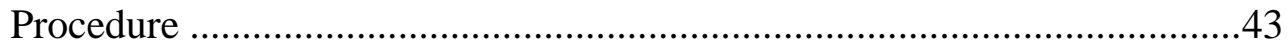

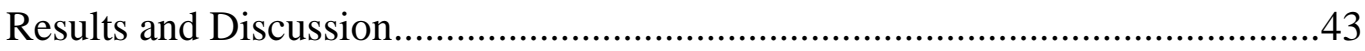

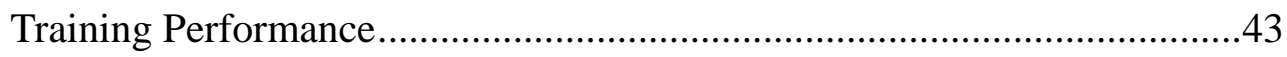

Transfer Performance ..........................................................................43

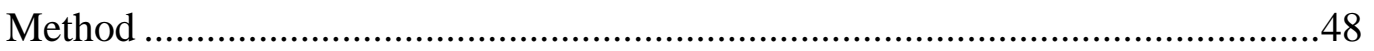

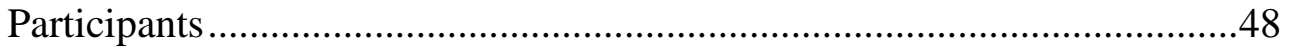

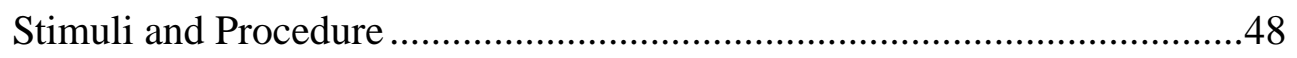

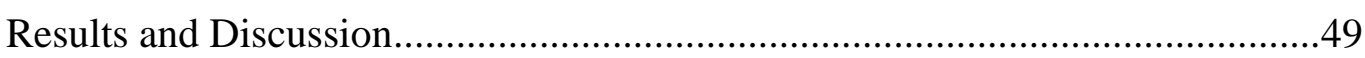

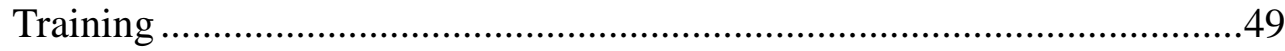

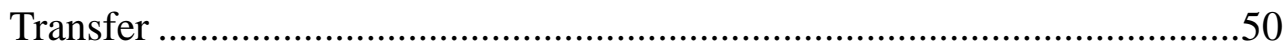

Modularization of classification rules .......................................................52

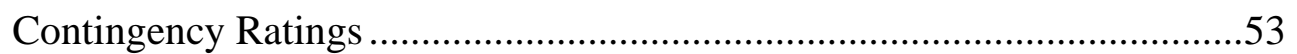

Chapter 5: Computational Models of Categorization ..................................................60

The Generalized Context Model (GCM) ................................................61

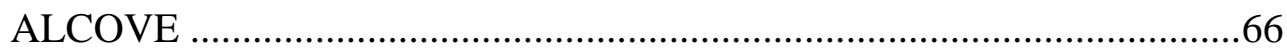

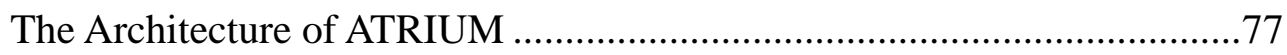

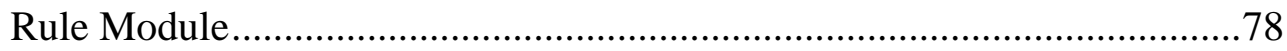

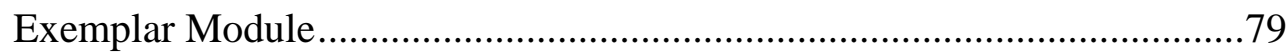

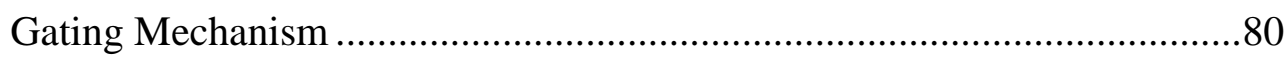

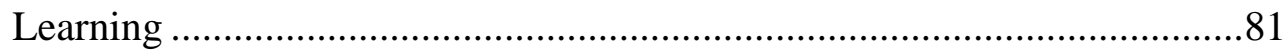

ATRIUM and Knowledge Partitioning ...................................................82

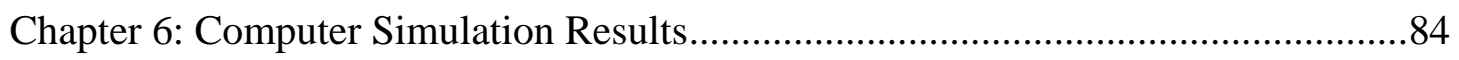

Modeling The Data From Experiment 1 and 2 ...............................................85

Fit of ALCOVE to the Randomized-Context Condition in Experiment

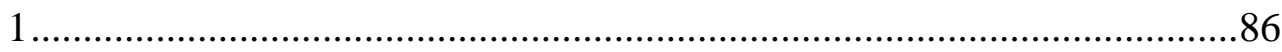

Fit of ALCOVE to the Systematic-Context Condition in Experiment 2......87

Modeling The Data From Experiment 5 .........................................................89

Fit of ALCOVE to the Randomized-Context Condition in Experiment 5

Fit of ALCOVE to the Systematic-Context Condition in Experiment 5......90 Individual Differences and ALCOVE ..............................................................91

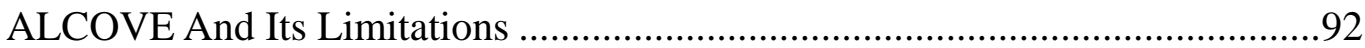

Modeling The Data From Experiments 1 and 2 .............................................93 
Fit of ATRIUM to the Randomized-Context Condition in Experiment 1....93

Fit of ATRIUM to the Systematic-Context Condition in Experiment 2.......95

Modeling The Data From Experiment 5 .............................................................96

Fit of ATRIUM to Randomized-Context Condition in Experiment 5..........96

Fit of ATRIUM to Systematic-Context Condition in Experiment 5 ............97

ATRIUM and Knowledge Partitioning ...........................................................100

Analysis of Weight Changes in ATRIUM ........................................................101

Weight Changes in The Exemplar Module ..............................................102

Weight Changes in The Rule modules ....................................................103

ATRIUM and Independence of Knowledge Parcels ..................................105

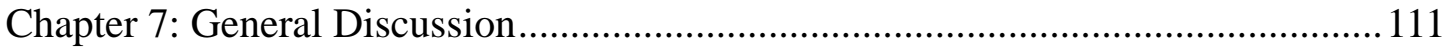

Complete Partitioning vs. Limited Heterogeneity ............................................. 113

Correlated Features in Categorization..........................................................113

Exemplar-Based Models of Categorization .....................................................119

Hybrid Models of Categorization..................................................................120

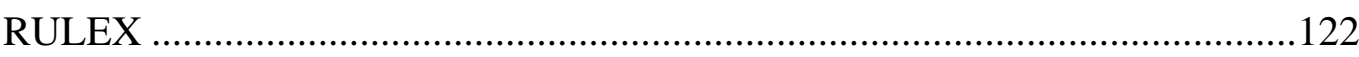

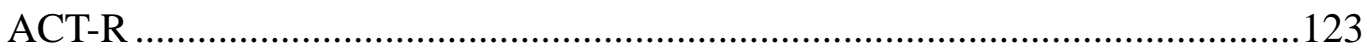

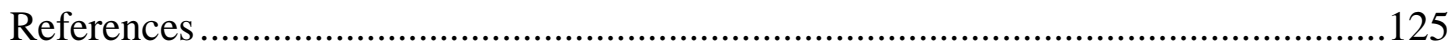

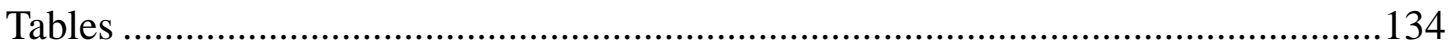

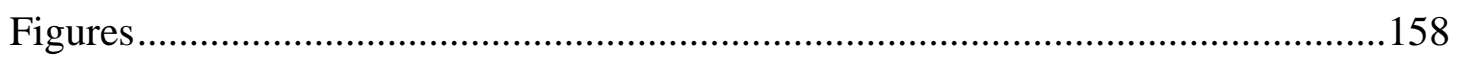




\section{Chapter 1: Knowledge Partitioning}

A good adaptation allows us to solve a problem encountered in the environment. For example, verbal communication is a problem for foreigners who do not speak the native language of a country. Therefore, an Asian person might be faced with a problem when accommodating him/herself in an anglophone country, unless he/she has acquired English language to an acceptable level. This example points out that we can be more competent at something, if we have gained specific knowledge about it.

Although the noun knowledge is common and familiar to us, the more deeply we think about it, the more confused we are becoming. What is knowledge? How is it structured or represented in our mind? Philosophers and psychologists have been interested in these questions for hundreds of years and the search for the answers is still continuing. Since knowledge cannot be touched by hands, tasted by tongue, or smelled by nose, a good way to uncover this secret in mind is via observing people's outer behavior. Specifically, comparing the performance of expert to that of novice can provide us a depiction of the structure of knowledge.

\section{Expert Knowledge and Knowledge Partitioning}

Experts can be identified by their outstanding performance in their domain, such as medicine, sport, chess and so on. It is commonly thought that experts have the ability to recognize key features of situations very quickly, thus allowing them to access information in memory that is relevant to those situations. In addition, experts have the ability to solve problems by efficient search in appropriate problem spaces (Newell \& Simon, 1972). Both kinds of abilities rest on the availability of a large, integral, and well-organized knowledge base, which is thus thought to be a characteristic of expertise (e.g., Ericsson, 1996; Glaser, 1996).

However, recent research has shown that expert knowledge is integral and well organized might not be the truth. Such a case was reported by Lewandowsky and Kirsner (2000). These researchers investigated the expertise of Australian bush fire fighters. Real fires are driven primarily by wind and slope of the terrain, such that fires tend to spread down-wind and up steep slopes. In certain situations, this means that fires will not always spread in the direction of the wind. Specifically, if the wind and the slope are directing a fire in opposite directions (i.e., the wind is 
blowing downhill), and the wind speed is slow and the slope is sufficiently steep, then fire will spread back into the wind and up the slope. Lewandowsky and Kirsner asked expert firefighters to predict the direction of fires under various wind and slope conditions, and found that when the wind and fire were in opposition — thus, creating uncertainty about the direction of the spread — experts only paid attention to partial aspects of the environment. However, the choice of variable that experts considered was determined by the context label co-presented with the stimulus fires. When a fire was presented as "To be Controlled" (that is, a fire that had been accidentally lit, or lit by arsonists, and was to be controlled), firefighters ignored any slope information and predicted the fire would spread with the wind. Conversely, when the same fire was presented as a "back burn" (i.e., a fire lit by fire fighters ahead of the main fire to starve it of fuel), experts ignored wind and predicted the fire would spread uphill. Thus, given identical physical situations (in which a fire will behave consistently), fire fighters predicted varying (and opposite) fire behavior, depending upon the context in which the fire was being considered.

Based on these contradictory predictions, Lewandowsky and Kirsner suggested that expert knowledge is not fully integrated, but separated into different parcels according to the context in which a problem is presented. Thus, in the case of the firefighters, Lewandowsky and Kirsner held that there were two parcels, one for the context of back-burn and the other for the context of firefighting, and the firefighters used different knowledge to judge an identical fire, depending on what context it is presented in. Lewandowsky and Kirsner called this theoretical concept “knowledge partitioning” (see Figure 1. of expertise, knowledge partitioning might therefore be a more generalized phenomenon in human knowledge acquisition and retrieval. This is evident that the phenomenon of "knowledge partitioning” can also be found in normal people. 
Knowledge partitioning is not restricted to experts. The important finding in the Lewandowsky and Kirsner (2000) study is that the expert firefighters made contradictory predictions in different contexts without awareness of the identical stimulus fire. This contextdependent employment of knowledge for solving problems is not exclusive to experts. Similar instances have been reported in the domain of "street mathematics" (e.g., Carraher, Carraher, \& Schliemann, 1985; Nunes, Schliemann, \& Carraher, 1993). Participants in those studies-Brazilian street vendors, carpenters, and cooks with minimal formal schooling—were sometimes shown to use different and contradictory ways to solve an otherwise identical problem in different contexts (Schliemann \& Carraher, 1993).

This finding implies that knowledge partitioning might be a fundamental process in acquisition and retrieval of knowledge even in non-experts. It follows that if we induce knowledge partitioning by experimentally manipulating the knowledge to be learned in laboratory, we can extend its theoretical contribution to the more basic human learning process.

\section{Laboratory Evidence of Knowledge Partitioning: Function Learning}

A first demonstration of knowledge partitioning in novice participants made use of the function learning task. In function learning experiments, people must learn the relationship between a continuous stimulus and a continuous response variable. For example, we may learn how long it will take us to stop a car as a function of the driving speed. Similarly, we may learn the relationship between the temperature in an oven and the time needed for roasting a chicken or between the rebound angle of a ball and the distance we should move to get it, and so on. In the laboratory experiments, participants are given a serials of training trials, on each of which they are asked to make a continuous response, which may be achieved by adjusting a bar's length according to a continuous stimulus, which may be represented as the length of another bar. Once participants make a response, they are given the correct response for feedback. Over trials, people learn the functional relationship between the continuous stimuli and response variables.

People can learn many different continuous functions when given sufficient practice on these discrete training trials (e.g., Busemeyer, Byun, Delosh, \& McDaniel, 1997). When the transfer stimuli fall within the range of training values, thus requiring interpolation, participants can predict 
responses very accurately (see Busemeyer et al., 1997, for a review). Participants can also extrapolate the learned function for responding to stimuli falling outside the trained range, although the accuracy is not as high as it is for interpolation (DeLosh, Busemeyer, \& McDaniel, 1997).

Using a U-shaped quadratic function that provided an analog to the fire prediction task, Lewandowsky, Kalish, and Ngang (2002) showed that people may acquire partitioned knowledge that is gated by the appropriate context. The stimulus was represented by the length of a stimulus bar and the response was the length of a response bar adjusted by participants. On each trial, participants were asked to predict the length of a response bar according to the length of the stimulus bar. The critical manipulation involved the presence of a context label during training that probabilistically identified the local relationship between stimulus and response magnitudes. That is, for some trials the context label "back-burn" would appear with the stimuli, whereas for others a context label of "fire-fighting” would appear. Specifically, 90\% of the stimuli with the stimulus length below the vertex of the quadratic function appeared with one context label (i.e., back-burn), whereas $90 \%$ of the stimuli whose stimuli lengths were above the vertex were presented in the other context. This manipulation created what Lewandowsky et al. called a "second-order" relationship between context and response magnitudes ${ }^{1}$. That is, owing to the symmetry of the quadratic function, context did not predict correct responses directly (i.e., no "first-order" relationship), as the mean response value was identical for stimulus magnitudes above and below the vertex. However, context did predict the functional relationship between stimulus and response. Another condition in their study was the randomized-context condition where the context appeared randomly during training, thus rendering context completely non-predictive.

Lewandowsky et al. (2002) observed context-specific transfer: When context appeared systematically during training, participants extrapolated along a different function in each context in preference to extrapolating along the U-shaped quadratic function. That is, in one context, participants applied a decreasing monotonic function for all transfer stimuli. Conversely, they applied an increasing monotonic function for all transfer stimuli in the other context. The

\footnotetext{
${ }^{1}$ The second-order relationship between context and stimuli is referred to the ability of context to identify a particular set of stimuli. For example, a stimulus presented with the context of "back-burn" stands for that
} 
decreasing monotonic function was formed by extending to the right side the left half of the Ushaped quadratic function, whereas the increasing monotonic function was formed by extending to the left side the right half of the quadratic function. However, participants in the randomizedcontext condition learned the true U-shaped quadratic function.

Of greatest interest in Lewandowsky et al. (2002) study is that participants in the systematiccontext condition learned to use a different function in a different context. Because context had no first-order relationship to responses, this result could not have occurred if context had directly contributed to a single weighted decision rule. Instead, Lewandowsky et al. argued that their results represented an instance of "knowledge partitioning"; that is, the creation during learning of independent parcels of knowledge that are selectively accessed on the basis of context. Further, owing to the dramatic difference of predicted response magnitude for the same diagnostic stimuli in different contexts, it was suggested that these two monotonic functions was used independently of each other. Applying this view, context "gated" access to a parcel of knowledge that was used to perform a task.

At the theoretical level, it is noteworthy that Kalish, Lewandowsky, and Kruschke (in press) presented a computational model of function learning that embodied these findings more formally. The model, known as POLE [Population of Linear Experts], can handle most existing data on function learning, including the partitioning effects reported by Lewandowsky et al. (2002). In addition, Kalish et al. (in press) demonstrated that partitioning a complex function into pieces of simple functions is a common strategy in function learning. In that case, Kalish et al. reported three experiments in which there was no context cue present and partitioning was based on the dimensional values of the stimuli. This finding suggests that any stimulus cue, not only some context cue, can be used as a basis for knowledge partitioning. Thus, knowledge partitioning might be fundamental to function learning.

POLE adopts the mixture-of-experts formalism (Jacobs, Jordan, Nowlan, \& Hinton, 1991). The mixture-of-experts structure includes multiple modules (or experts), which can independently learn to predict the outcomes, and the final outcome of the system results from each module's 
prediction weighted via a gating mechanism. Accordingly, POLE has multiple modules for learning a function, but the modules compete for a response on each trial. Each module can learn a linear function with a constant intercept and slope, and across the population of modules, a large variety of slopes and intercepts are represented. It follows that POLE partitions any but the simplest linear function by associating different subsets of stimuli to different partial functions; for example, a quadratic function is learned by piecing together a number of linear modules that are associated to different sets of stimulus magnitudes. Specifically, once a candidate module is chosen, knowledge in other modules is ignored-this exclusivity of access may be an essential feature of any theory capable of handling the growing body of evidence for knowledge partitioning. This is different from other mixtures-of-experts designs (e.g., ATRIUM [Attention To Rules and Instances in a Unified Model]; Erickson \& Kruschke, 1998, 2001) in which the system response is generated by averaging across the responses of all modules.

\section{Knowledge Partitioning is a Phenomenon As Well As a Theory}

To date, knowledge partitioning has been evident in experts' and novices' knowledge acquisition (Lewandowsky et al., 2002; Lewandowsky \& Kirnser, 2000). It is also evident in the circumstances where no binary context cue is present, but instead the stimuli themselves are used, as a basis for partitioning (Kalish et al., 2003). Thus, there is no disagreement with that knowledge partitioning is an important phenomenon in acquisition of knowledge. The main assumptions of the theory of knowledge partitioning include (1) that with the aid of partitioning the to-be-learned knowledge into pieces by context or other stimulus information, people can learn complex functions (or knowledge) more easily (e.g., Lewandowsky et al., 2002; Kalish et al., 2003) and (2) that when people use one knowledge parcel, they seem not to be aware of the existence of other parcels ${ }^{2}$. As indicated the second assumption, it is not adequate to call the contextualized psychological processing (i.e., contextualized categorization) as knowledge partitioning. In fact, the spirit of knowledge partitioning is that in our mind, the knowledge in a specific domain might be divided into separate substructures by context (or stimuli themselves), although it should be organized as an integrated entirety with inner coherence. To capture these characteristics of

\footnotetext{
${ }^{2}$ Otherwise, the bush fire experts in the study of Lewandowsky and Kirsner (2000) would have noticed the contradictory decisions they made.
} 
knowledge partitioning, the mixture-of-experts framework is crucial. This is because what is contained in each expert represents for the partitioned knowledge. However, the way to use those experts is as implemented in POLE.

Due to the success of exposing knowledge partitioning in function learning, it follows that if knowledge partitioning can occur in other learning tasks, such as category learning, then a model employing the mixture-of-experts architecture might perform better than a model without it in accounting for knowledge partitioning. A principal purpose of this research is to explore that expectation.

Chapters 2, 3 and 4 report six experiments which support knowledge partitioning in the category learning paradigm. These positive findings not only extend the applicability of knowledge partitioning, but also create challenges to current categorization theory. Hence Chapter 5 discusses some potentially appropriate categorization models for the observed data. In Chapter 6, two neural network models' capacities to account for knowledge partitioning are compared. Chapter 7 is the general discussion and conclusion. 


\section{Chapter 2: Conceptual Category Learning (I)}

\section{Why Choose Categorization to Extend Understanding of Knowledge Partitioning?}

First, categorization is crucial to our survival and important in the cognitive domain, as stated by Estes (1994): “Memory is essential to adaptive behavior because it is organized in ways that make information gained from past experience applicable to present situations. And the essence of memory is classification (p 4)”.

Our reaction to events that we encounter in our everyday lives is often classificationdependent. For example, we would probably behave one way if told a tiger was approaching, and another way if we were told a household cat was approaching. This is because our knowledge or concepts of different categories (i.e., tiger vs. household cat) guides our behavior to fit the environment. Similarly, when we feel sick and go to see a doctor, the doctor assigns our symptoms to a known disease, in order to decide on the appropriate medical treatment. Thus, categorization is the process which maps an observed object, based on its features (i.e., symptoms), to a category (i.e., disease). Thereafter, according to the knowledge associated with the category it belongs to, we can suitably react to that object. This characteristic of categorization allows us to apply the same reaction to different objects, if they are classified in the same category, which in turn reduces our cognitive load (e.g., Neisser, 1987; Smith \& Medin, 1981). Therefore, categorization is essential to our survival.

Second, the process of category learning is similar to function learning. In function learning, people learn to map continuous stimuli (e.g., the outdoor temperature) to continuous responses (e.g., the duration for watering the lawn), whereas in category learning (such as learning to map symptoms to different diseases), the mapping is established between (often discrete) stimuli and discrete category responses (Busemeyer et al., 1997). Since knowledge partitioning can be induced in function learning (e.g., Kalish et al., in press; Lewandowsky et al., 2002), it may occur in category learning as well.

Third, there is no research to date attempting to combine knowledge partitioning with category learning. An obvious application of examining knowledge partitioning in 
Chapter 2: Conceptual Category Learning (I)10

categorization is to provide novel findings to evaluate the current categorization theories. Before describing the experiments in this research, some issues in category learning which might be relevant to knowledge partitioning will be introduced.

\section{Category Learning}

The fundamental question in the field of category learning is concerned with how people make a classification decision. The classic view (e.g., Bourne, 1970) assumes that an instance is assigned to a category by analyzing its constituent features. For example, an object will be classified as a "triangle" if it possesses the necessary features "has three sides and interior angles sum to $180^{\circ}$ ”. However, this hypothesis-testing procedure is incompatible with research on natural categories (i.e., bird, tree etc). This is because natural category structures are fuzzy and no sets of features can define natural categories without exceptions (Rosch \& Mervis, 1975). As a result, the classic hypothesis-testing account (e.g., if one object has feature A, it must be category 1) was not considered appropriate for investigating categorization. Subsequently, the direction of research from the mid-1970s to the present has turned towards the stored mental representations of categories (Estes, 1993).

\section{Category Representation}

What is it that is actually stored in our memory as the category representation? Recent research to answer this question has adopted three different approaches: (1) The rule-based view holds that after learning categories, a rule is stored in our head for categorization, so if an object has a feature value $\mathrm{X}$ that exceeds a criterion value $\mathrm{X}_{\mathrm{c}}$, then the object is classified in one category, and otherwise it is placed in the other (e.g., Ashby \& Gott, 1988; Ashby \& Maddox, 1992; Ashby \& Townsend, 1986; Maddox \& Ashby, 1993). For example, a student will be judged as having "passed” if his/her score is higher than 50\%, otherwise they will have "failed”. (2) According to the instance view, all experienced exemplars are stored in our memory for categorization, such that an object is classified into one category if its summed similarity to the stored exemplars from that category is larger than the other (e.g., Kruschke, 1990, 1992a, 1993; McKinley \& Nosofsky, 1995; Medin \& Schaffer, 1978; Nosofsky, 1984, 1986, 1987, 1988a, 1988b; Nosofsky, Clark, \& Shin, 1989; Nosofsky \& 
Chapter 2: Conceptual Category Learning (I)11

Johansen, 2000; Nosofsky \& Kruschke, 1992; Nosofsky, Kruschke, \& McKinley, 1992; Nosofsky \& Palmeri, 1997; Shin \& Nosofsky,1992; Zaki \& Nosofsky, 2001); and (3) Recent hybrid views hold that both the rule and the exemplars are stored after categories have been learned (e.g., Anderson \& Betz, 2001; Erickson \& Kruscke, 1998, 2001, 2002; Nosofsky \& Palmeri, 1998; Nosofsky, Palmeri, \& McKinley, 1994; Vandierendonck, 1995). Different manners of employing the rule and the exemplars are assumed in different hybrid models, such as that the exemplars are used for classifying those exceptions to the rule or that both the rule and the exemplars are used together for classifying each item, and so on. Despite their different stances on the use of both representations, the hybrid views are distinguished from the other views in terms of the assumption of both the rule and the exemplars as category representations. Most of the current theories of categorization hold one of these perspectives, each of which has supporting evidence.

Which of these theories is preferred overall is still being debated, and a more diagnostic examination is required to distinguish them. The examination of knowledge partitioning in categorization may be able to do this. This is because none of the perspectives has ever made explicit arguments about the possibility of category representation being gated by an irrelevant context. Recall that in knowledge partitioning, knowledge representations are stored in independent parcels and retrieved by context that has no direct predictive relationship to the outcome. This permits the existence of heterogeneous representations in a psychological space. Thus, a model which cannot deal with heterogeneous representations might have difficulty accounting for knowledge partitioning.

For example, the rule-based model (e.g., GRT [General Recognition Theory]; Ashby \& Gott, 1988) might not be able to account for knowledge partitioning. This is because in the GRT, all stimuli in a psychological space are assumed to be classified by a decision boundary and there is no argument for the GRT to have heterogeneous boundaries in different contexts. For the exemplar-based model (e.g., ALCOVE [attention learning covering map]; Kruschke, 1992; GCM [General Context Model]; Nosofsky, 1984, 1986), it is unclear whether or not it can account for knowledge partitioning. Although the stored exemplars contain all information for categorization, all exemplars are assumed to be 
Chapter 2: Conceptual Category Learning (I)12

represented in a unified psychological space. Perhaps, the hybrid models may have a greater chance to account for knowledge partitioning, given that at least those models employ different types of representation (i.e., rule and exemplar). Among the current hybrid models of categorization, ATRIUM (Attention To Rules and Instances in a Unified Model; Erickson \& Kruschke, 1998) deserves most emphasis. This is not only because ATRIUM employs both rules and exemplars as representations, but also because it adopts the mixture-of-experts architecture which is thought to be the key point to account for knowledge partitioning in function learning. Given that both the exemplar-based model and the hybrid model may be able to account for knowledge partitioning, in Chapter 6, the computer simulation focuses on the comparison between two models, ALCOVE (an exemplar-based model) and ATRIUM (a hybrid model). Thus, should knowledge partitioning occur in categorization, it can be a touchstone for testing the capacity of different theories of categorization. Therefore, in addition to extending knowledge partitioning to a category learning paradigm, this research will also make a theoretical contribution to categorization research.

\section{$\underline{\text { Heterogeneous Category Representations }}$}

The possibility that the stored category representation can be gated by context has not been investigated before, yet some relevant precedents exist. First look at the natural categories. The structural principle underlying a natural category is thought to be the family resemblance principle (Rosch \& Mervis, 1975). That is, for a given category, each instance shares at least one feature in common with one or more instances, but no feature is common to all instances (Rosch \& Mervis, 1975). It follows that the more features an instance has in common with other instances, the more typical that member is of its category. For example, a pigeon is a more typical bird than a goose. This typicality effect is demonstrated by higher typicality ratings and shorter reaction times for more typical items, when people are asked answer the true-or-false questions like "a pigeon is a bird” or "a goose is a bird” (Rips, Shoben, \& Smith, 1973).

Not only does the number of features shared with other instances influence a subject's typicality rating, the context also influences people’s behavior. For example, Roth and Shoben (1983) showed that people judged milk as a more typical beverage than tea when a 
truck driver (i.e., context) was thought to be consuming the beverage, however, the typicality judgement reversed when a secretary was the subject in the question. This is evidence against an invariant semantic space and in favor of a more heterogeneous knowledge landscape. Medin and Shoben (1988) reached a similar conclusion using typicality judgments of adjective-noun combinations. For example, when judging the pair-wise similarity between black, gray, and white, participants considered black and gray to be more similar than white and gray in the context of clouds, but this judgment was reversed in the context of hair $\equiv \mathrm{r}$.

Similarly fluid performance has been observed in category learning with artificially constructed stimuli. For example, Lamberts (1994) presented exemplars from a hypothetical family defined by features such as size of ears or eyes, or presence vs. absence of hair. Participants then had to determine the family membership of a target person that was presented either as a brother or cousin of one of the training exemplars. Lamberts found that people applied a more rigorous inclusion criterion in the "brother" context (i.e., a brother must be very similar to other members of his family) than in the "cousin" context.

Even stronger evidence for representational heterogeneity was provided by Aha and Goldstone (1992). In their experiment, training stimuli were sampled from two distinct clusters in a two-dimensional category space, each bisected by its own uniquely oriented boundary. Because the design of the Aha and Goldstone study is relevant to the experiments reported in this thesis, the stimulus space from their Experiment 1 is presented in Figure 2. Training items are identified by their category membership (either A or B), and transfer items are labeled $\mathrm{X}, \mathrm{Y}, \mathrm{Z}$ and $\mathrm{W}$. Stimuli varied along two perceptual dimensions, the size of a rectangle and the horizontal offset of a vertical line segment within it, with 8 possible levels for each dimension. Correct classification of the training instances in the top-right cluster can be achieved by locating a vertical linear boundary in-between 6 and 7 on the abscissa, whereas the bottom-left cluster can be correctly classified by a horizontal boundary in-between 6 and 7 on the ordinate. Note that the two boundaries are globally incompatible because, when extended further from their cluster, they dictate opposite classifications for the same test items. 
Aha and Goldstone found that people classified the critical transfer stimuli X and Y predominantly as belonging to category A and B, respectively, which means that people applied the vertical boundary to Y but the horizontal boundary to X. This shows that people can be sensitive to different patterns of features formed by a subset of stimuli, and may apply different strategies to each subset. Aha and Goldstone furthermore showed that the data could only be handled by a model that incorporated unique dimensional weights for each exemplar. Specifically, Aha and Goldstone modified Nosofsky’s GCM (e.g., 1986) by inserting a weighting function into the computation of similarities between exemplars. The function incorporated unique learned dimensional attention weights for each exemplar, thus creating a heterogeneous internal category representation.

Erickson and Kruschke (2001) recently reported a replication and extension of the Aha and Goldstone (1992) study. In particular, Erickson and Kruschke (2001) showed that the observed heterogeneity was not an artifact of averaging across subgroups of participants who had mastered only one, but not the other, of the training clusters. Erickson and Kruschke also supported Aha and Goldstone’s theoretical conclusion, namely that the data required a stimulus-dependent representation that is difficult to account for by most current models.

In summary, there is evidence that the representations of natural and artificial categories can be quite heterogeneous.

\section{$\underline{\text { Partitioning Knowledge in Categorization }}$}

In Aha and Goldstone’s (1992) study, the critical transfer stimuli X and Y were classified according to the boundary within the nearest cluster of training items (see Figure2.0). This arguably reflected some partitioning of knowledge because categorization depended on the immediate neighborhood of a transfer item, an assertion supported by the 
exemplar-specific dimensional weights required to model the data. However, at the same time, participants were sensitive to the global incompatibility of the two local boundaries, because generalizations from the training items were limited to the immediate vicinity of each cluster. For example, item X was not classified as B despite this being suggested by the nearby vertical boundary. It follows that although people's knowledge was sensitive to the idiosyncracies of each cluster, and hence heterogeneous, it was also sensitive to the presence of the other cluster.

The function learning results of Lewandowsky et al. (2002), by contrast, revealed that people may selectively access knowledge in one parcel without considering information demonstrably accessible in another context. In terms of Aha and Goldstone’s (1992) design, this would be tantamount to showing that people generalize from each cluster of training items on the basis of information contained in that cluster alone, without regard to what they have learned about the other cluster, and without awareness of the global incompatibility of the two local decision boundaries.

In this chapter, two experiments are reported that sought evidence for such partitioning of knowledge in category learning. The methodology was inspired by the function learning design of Lewandowsky et al. (2002); hence, a binary context label was used to manipulate and encourage knowledge partitioning. To facilitate presentation, I report and interpret the results from a rule-oriented perspective and identify the rules used by participants in each experiment (cf. Lewandowsky, Kalish, \& Griffiths, 2000; Nosofsky et al., 1994). Discussion of the various theoretical alternatives is deferred to Chapter 4 which is the final chapter to report data.

\section{Experiment 1}

All experiments reported in this thesis sought evidence for the existence of knowledge partitioning as defined by five criteria: (1) Categories were separated by a single but complex boundary that could be partitioned into simpler components. (2) The simple component boundaries had to be identifiable by a context variable during training, and (3), context could not be a valid direct predictor (i.e., context had to be a "second-order" but not 
Chapter 2: Conceptual Category Learning (I)16

"first-order” predictor). (4) The complex boundary had to be learnable when context was either absent or had no predictive value (i.e., was not even a second-order predictor). Finally, (5) there had to be a diagnostic set of transfer stimuli that could help determine whether or not knowledge partitioning emerged during training. These 5 criteria were satisfied as follows.

The principal between-subjects manipulation concerned the role of context, thus there were two conditions in Experiment 1: the randomized-context condition and the systematiccontext condition. In the randomized-context condition, context labels were randomly assigned to training items and thus had no predictive value. This served to examine criterion (4), that people can learn the complex boundary unless partitioning is encouraged. Partitioning was encouraged in the systematic-context condition, in which context was consistently mapped not to an outcome but to subsets of training items, each characterized by a component boundary. This manipulation satisfied criteria (2) and (3) above. (The systematic-context condition introduced a correlation among predictors within a category. Discussion of previous research on correlated predictors is deferred until after presentation of all results.) Both conditions used the same set of transfer items that were tested in both contexts. This permitted identification of knowledge partitioning (criterion 5) through diagnostic response patterns that are described below.

\section{$\underline{\text { Method }}$}

\section{$\underline{\text { Participants and Apparatus }}$}

Sixty-two volunteers from the University of Western Australia campus community participated voluntarily in exchange for partial course credit. An equal number of participants was randomly assigned to each condition.

The experiment was controlled by a PC that presented all stimuli and collected and scored all responses. The same apparatus was used in all experiments.

\section{$\underline{\text { Stimuli }}$}

All stimuli were sampled from the two-dimensional pseudo-continuous category space shown in Figure 2. $\equiv$ he bilinear category boundary, represented by the solid line, is 
Chapter 2: Conceptual Category Learning (I)17

described by: $\mathrm{Y}=500-|\mathrm{X}-400|$. Stimuli below and above the triangular boundary belonged, respectively, to category A and B. To clarify notation, from here on I use "boundary" to refer to a design feature of the stimulus space and I use "rule" to refer to participants’ imputed categorization strategy.

Insert Figure 2.2 about here

The two categories were arbitrarily instantiated as different species of fish, with predictors X and Y labeled as “density” and “depth,” respectively. Participants were told that density referred to the amount of food available in the habitat, whereas depth referred to how far below the surface the fish preferred to live.

Training items. All participants were trained on a common set of 40 stimuli, all with Y $<500$. Training stimuli were separated into two subsets of 20 with values of $X$ above and below 400. Each subset contained an equal number of instances of each category which clustered around the local diagonal boundary whose orientation was positive for $\mathrm{X}<400$ and negative for $X>400$. Training instances belonging to category A are represented by open circles in Figure 2.2 and those belonging to category B by crosses. The training sequence consisted of 10 blocks, each of which involved presentation of all 40 items in a different random order.

Each stimulus also included a third, binary context feature (C), not shown in the figure. Regardless of condition, both levels of context $\left(\mathrm{C}_{1}\right.$ and $\left.\mathrm{C}_{2}\right)$ were equally represented among training instances and categories. Context therefore was not a direct (i.e., "first-order”) predictor. In the randomized-context condition, context also had no second-order relationship because it was randomly paired with training instances, subject to the constraint that each context had to occur equally often with each category. In the systematic-context condition, one level of context (e.g., $\mathrm{C}_{1}$ ) occurred with stimuli whose value of $\mathrm{X}$ was below 400, whereas the other level (e.g., $C_{2}$ ) occurred with values of $X>400$. Hence there was a perfect correlation between $\mathrm{C}$ and the relationship between $\mathrm{X}$ and $\mathrm{Y}$ that defined the local boundary between categories $\mathrm{A}$ and $\mathrm{B}$. 
Chapter 2: Conceptual Category Learning (I)18

Context was instantiated as "season” using the two levels "summer” and "winter." Assignment of context labels to magnitudes of $\mathrm{X}$ was counterbalanced between participants; for ease of exposition, from here on I refer to context as "left" and "right" to represent whichever label accompanied values of X below and above 400, respectively.

Transfer items. The 20 transfer stimuli are identified by filled diamonds in Figure 2.2. There were two blocks of transfer trials, each consisting of a different random order of all transfer stimuli. Context alternated between blocks for each transfer stimulus, and each context was represented equally often in each block.

Diagnosticity of transfer items. Transfer items were sampled from the 4 areas identified by number in Figure 2.2. The expected pattern of responses differs between and within areas depending on whether or not people partitioned their knowledge.

If partitioning is absent, then the true boundary should apply in both contexts, and all items from Area 1 should be consistently classified as belonging to category A and items from the remaining areas (2-4) as belonging to category B. A very different pattern is expected for the systematic-context condition if people partition their knowledge. In that case, one would expect the positive diagonal component of the boundary to be used to classify all stimuli in the "left" context. This extended partial rule is indicated by the dotted lines along the positive diagonal in Figure 2. Accordingly, in the "left" test context, stimuli in Areas 2 and 3 should be predominantly classified as belonging to category B, whereas those in Areas 1 and 4 are expected to be classified as A. The reverse situation is expected in the "right” context. In that case, the negative partial boundary is expected to be used (also indicated by a dotted line in the figure) and stimuli in Areas 1 and 2 would be classified as A, whereas those in Areas 3 and 4 would be classified as B. Overall, knowledge partitioning would be identified if responses differ between test contexts for Areas 2 and 4 but remain the same in Areas 1 and 3.

\section{Procedure}

Participants were tested individually in a quiet booth. On every trial, an unchanging schematic icon of a fish was displayed on the screen. Below the icon, the three labels density, depth, and season were displayed from left to right, with the corresponding feature values 
displayed immediately underneath. Categorization responses were recorded using the " $\mathrm{f}$ " and “j” key. Assignment of categories to keys alternated across participants. Each trial commenced with display of a fixation signal (a “+”) in the center of the screen for $500 \mathrm{~ms}$, followed by presentation of the stimulus which remained visible until participants responded. In the training phase, participants were given feedback (the word "correct" or "wrong" shown in the center of the screen) for $1000 \mathrm{~ms}$ after each response before the next trial commenced. No feedback was presented during transfer.

Self-paced breaks were inserted after every 40 training trials and after every 20 transfer trials. Additionally, there was a break between training and transfer. The experiment lasted just under $1 \mathrm{hr}$.

\section{$\underline{\text { Results and Discussion }}$}

\section{Training Performance}

Training performance was analyzed using the proportion of correct responses in each block as the dependent measure. A 2 (Condition) $\times 10$ (Training Block) between-within analysis of variance (ANOVA) revealed a significant main effect of Block $[\underline{F}(9,540)=$ 51.193, MSE $=.009, \underline{\mathrm{p}}<.001]$, along with a marginal main effect of Condition $[\underline{\mathrm{F}}(1,60)=$ 3.889, MSE $=0.103, \underline{p}=.053]$. The interaction between both variables was not significant with $\underline{\mathrm{F}}(9,540)<1$. The main effect for Block resulted from an improvement in performance from the first ( $\underline{M}=.552)$ to the last block of training $(\underline{M}=.797)$, whereas the main effect of Condition reflected the fact that people were better overall in the systematic-context condition ( $\underline{\mathrm{M}}=.726)$ than in the randomized-context condition $(\underline{\mathrm{M}}=.675)$.

The latter effect replicated a related finding by Lewandowsky et al. (2002) who also found that performance during training was better for the systematic-context condition. I suggest that this reflects the enhanced ease of the task when it can be partitioned into independent sub-components on the basis of context. I next show that this type of partitioning occurred in Experiment 1.

\section{Transfer Performance}


Chapter 2: Conceptual Category Learning (I)20

The dependent variable for all transfer analyses in this thesis was the probability of classifying a stimulus as belonging to category A. Responses were aggregated across transfer items within each of the four diagnostic areas. Table 2. for each condition and area.

Insert Table 2.1 about here

A 2 (Condition) $\times 2$ (Test Context) $\times 4$ (Area) between-within ANOVA revealed a main effect of Area $[F(3,180)=76.12$, MSE $=0.12, \mathrm{p}<.01]$, and two two-way interactions involving Test Context and Area $[\mathrm{F}(3,180)=8.47$, MSE $=0.04, \mathrm{p}<.01]$, and Condition and Test Context $[\mathrm{F}(1,60)=6.67, \mathrm{MSE}=0.04, \mathrm{p}<.05]$. These effects were qualified by an over-arching interaction involving all three variables, with $\underline{F}(3,180)=5.17, \operatorname{MSE}=0.04, \underline{p}$ $<.01$. No other effects were significant, with the largest $\underline{\mathrm{F}}=2.19(\mathrm{p}>.10)$.

The crucial three-way interaction was first explored by computing a separate Test Context $\times$ Area effect for each condition, which was found to be significant in the systematic-context condition $[\underline{F}(3,90)=8.95, \operatorname{MSE}=0.06, \mathrm{p}<.01]$ but not in the randomized-context condition $[F(3,90)=1.22]$. Further exploration involved simple comparisons between test contexts for all areas in the systematic-context condition. These tests revealed no significant effect in Area $1[\underline{F}(1,30)=2.06$, MSE $=0.02, \underline{p}>.10]$, but significant effects in Area $2[\underline{\mathrm{F}}(1,30)=11.14, \mathrm{MSE}=0.09, \underline{\mathrm{p}}<.01]$, Area $3[\underline{\mathrm{F}}(1,30)=$ 10.87, MSE $=0.06, \underline{\mathrm{p}}<.01]$, and a nearly significant effect in Area $4[\underline{\mathrm{F}}(1,30)=3.73, \mathrm{MSE}$ $=0.07, \underline{p}=0.063]$.

Knowledge partitioning in categorization. These results suggest a straightforward interpretation: First, people were clearly able to learn the correct bilinear boundary when context was of no predictive value. In the randomized-context condition, people correctly classified transfer items below the bilinear boundary as belonging to category A, whereas all others—including those in Area 3 furthest from the trained region—were predominantly classified as belonging to category B. Second, when context predicted the orientation of a local boundary, there was some suggestion that people learned to partition their knowledge. 
Transfer performance was sensitive to context in Areas 2 and 4 (but not Area 1), as would be expected when partial rules are used.

However, these conclusions are accompanied by two limitations. The first involves the apparent asymmetry of the partial rules. The second concerns the magnitude and consistency of the effect.

Asymmetry of partitioning. If people in the systematic-context condition consistently applied one or the other partial rule, the pattern of responding in Areas 1 and 3 should remain unaffected by test context. The data partially contradict this expectation: Although there was no effect of context for stimuli in Area 1, there was an unexpectedly large and significant effect in Area 3.

This unexpected result is explored further in Figure 2. a category A response, averaged across participants, for each transfer item in the systematiccontext condition separated by test contexts. The top panel ("left” test context) suggests that a partial rule was applied with a fair amount of consistency to most test items (with the exception of at least one item, in the lower right, for which P(A) was only .29 despite it being in the region in which items should be classified as belonging to A.) By contrast, the bottom panel (“right” context) does not suggest use of a partial rule. Instead, the data are more consistent with (imperfect) use of the correct bilinear boundary. This asymmetry among test contexts is reminiscent of the known characteristic of function learning that ascending functions are easier to learn than descending functions (e.g., Busemeyer et al., 1997; Lewandowsky et al., 2002).

Insert Figure 2.3 about here

Magnitude of partitioning: Individual differences. The magnitude of the context effect for Areas 2 and 4 is not indicative of a consistent application of partial rules. Consistent knowledge partitioning by all participants would imply probabilities close to 0 and 1 across the two contexts. This was not observed. For example, in the left context, P(A) for Area 4 
was .44, which is close to what would be expected by chance or if different individuals applied different strategies.

Participants’ individual differences were explored by entering participants’ response profiles (i.e., the pattern of a participant's A vs. B responses to all transfer stimuli) into a $\underline{\mathrm{k}}-$ means cluster analysis that used four pre-defined clusters (cf. Lewandowsky et al., 2000). Cluster centroids were pre-defined to represent application of the (1) correct bilinear boundary, (2) the left partial boundary, (3) the right partial boundary, and (4) a horizontal boundary at $Y=300$. Using a Euclidean distance measure, the analysis assigned each participant's response profile to the closest cluster. Participants who were equidistant from all predefined $\equiv$ erns were considered to be unrecognizable.

Table 2. $\equiv$ ows the assignment of participants to clusters for each condition and test context. Confirming the overall transfer analysis, the top panel of the table shows that there was little or no effect of context in the randomized-context condition. The results in the systematic-context condition differed in several important ways: First, fewer participants overall applied the correct bilinear boundary. Second, there was a notable effect of context for the partial rule clusters, with a significant proportion of participants using the partial rule that was appropriate to the context. Third, fewer participants overall applied the descending partial boundary than the ascending one; this again confirms that negative relationships are more difficult to learn than positive $\equiv$.

\section{Insert Table 2.2 about here}

One problem concerns the large percentage of participants who remained unrecognizable. Two related reasons can be cited for this: First, there were few stimuli in the generalization regions (i.e., with $\mathrm{Y}>500$ ), which made differentiation of some candidate rules more difficult. Second, there were two transfer stimuli (one in Area 2 and one in Area 4) that were in close proximity to the centroid of studied items belonging to category B. The similarity of those test stimuli to studied exemplars may have overridden the application of a partial rule that dictated an opposing classification (e.g., Nosofsky, et al., 1989). 
This problem was resolved by Experiment 2, which used the same category space but ensured that transfer items were further away from the trained stimuli. Experiment 2 also included two control conditions to help identify the extent of knowledge partitioning.

\section{Experiment 2}

The category structure used in this experiment is shown in Figure 2. not defined.. It differs from the previous one only with respect to the location of transfer items. In this experiment, the main purposes were to replicate knowledge partitioning avoiding the influence of the centroids of the categories and to examine the independence of partitioned knowledge.

The randomized-context condition was not included in Experiment 2, because Experiment 1 showed that people could learn the bilinear boundary when no cues are available for partitioning. In addition, Experiment 2 included two single-context learning conditions in order to examine the independence of knowledge parcels. In the left-only condition, only the "left" set of instances (i.e., those with $X<400$ ) was presented for training in a single context. In the complementary right-only condition, participants learned the "right” set of instances $(X>400)$. Comparison of transfer performance in these two singlecontext conditions to performance in the matching test context of the systematic-context condition can reveal the extent of partitioning. Specifically, should transfer performance be identical between conditions within each context, the two partial knowledge components acquired in the systematic-context condition could be considered largely independent. Conversely, should those comparisons reveal an effect of condition within each context, this would identify at least some linkage between knowledge components.

Insert Figure 2.3 about here

\section{Method}

\section{$\underline{\text { Participants }}$}


Chapter 2: Conceptual Category Learning (I)24

Sixty-three undergraduates from the University of Western Australia participated voluntarily in exchange for partial course points. Thirty-one participants were randomly assigned to the systematic-context condition and 16 each to the left-only and right-only conditions.

\section{$\underline{\text { Stimuli and Procedure }}$}

The systematic-context condition used the 40 learning instances from Experiment 1. In the left-only condition, participants learned the 20 instances located in the left half of the stimulus space with a single context cue (counterbalanced across participants). Conversely, training in the right-only condition involved the other 20 learning instances. There were 10 blocks of training trials in all conditions. Each block contained a different random sequence of the training items.

The transfer test was identical for all conditions and involved a novel set of 20 stimuli that were further removed from training items (see Figure 2.3). The remaining details of the transfer test and the procedure were unchanged from Experiment 1. This implied that half the transfer stimuli in the left-only and right-only conditions were presented in a completely new context not encountered during training.

\section{$\underline{\text { Results and Discussion }}$}

\section{$\underline{\text { Training Performance }}$}

The probability of correct responses across blocks increased from .64 (Block 1) to .84 (Block 10). Performance differed considerably between conditions, with the systematiccontext condition $(\underline{\mathrm{M}}=.70)$ being worse overall than the left-only ( .88$)$ and right-only conditions (.76). This difference between conditions was not unexpected because the latter condition included twice as many learning instances as each of the two single-context conditions. The single-context advantage may have been further enhanced by the greater complexity of the bilinear category boundary in the systematic-context condition. In addition, the superiority of the left-only over the right-only condition again shows that the ascending partial boundary was easier to learn than its descending counterpart. 
These impressions were confirmed by a 3 (Condition) $\times 10$ (Block) between-within ANOVA that revealed main effects for Block $[F(9,540)=23.8, \operatorname{MSE}=0.01, \mathrm{p}<.01]$ and Condition $[\mathrm{F}(2,60)=10.307, \mathrm{MSE}=0.17, \mathrm{p}<.01]$ but no interaction between both variables $[F(18,540)<1]$. Exploration of the main effect of Condition (by Dunnett's test) revealed no differences between the systematic-context condition and the right-only condition, but a consistent difference in all blocks between the systematic-context and left-only conditions. The corresponding pairwise differences between the left-only and right-only conditions were significant or marginally significant in Blocks 4 through

\section{Transfer Performance}

Knowledge partitioning. I first determined whether or not knowledge partitioning was present by focusing on the systematic-context condition. The average probability of category A responses in the four areas of interest are shown in Table 2.1.

A 2 (Test context) $\times 4$ (Area) within-subjects ANOVA revealed significant main effects of Context $[\underline{F}(1,30)=5.01$, MSE $=0.05, \underline{p}<.05]$ and Area $[\underline{F}(3,90)=25.08$, MSE $=0.12, \underline{p}$ $<.01]$, as well as a significant interaction between both variables $[\underline{F}(3,90)=11.55$, $\operatorname{MSE}=$ $0.08, \mathrm{p}<.01]$. Simple comparisons between the two contexts in all four areas were significant for Area $2[\underline{F}(1,30)=18.14, \operatorname{MSE}=0.08, \underline{p}<.001]$, Area $3[\underline{F}(1,30)=7.87$, MSE $=0.08, \underline{p}<.01]$, and Area $4[\mathrm{~F}(1,30)=10.44, \operatorname{MSE}=0.07, \mathrm{p}<.01]$. The comparison was non-significant only for Area 1 , with $\underline{\mathrm{F}}(1,30)=1.09$.

As in the first experiment, the context effects in Areas 2 and 4 were indicative of the presence of knowledge partitioning, whereas the significant effect for Area 3 was again unexpected. One possible reason underlying the effect in Area 3 was that the descending partial boundary was harder to learn than the ascending one. This possibility can be examined by comparing the right-only and left-only conditions. Figure 2. probabilities for all transfer items presented in the trained context in the left-only (top panel) and right-only (bottom) conditions. For the left-only condition, it is clear that people learned the partial boundary involving the positive diagonal quite well, because all items below that boundary are classified with near uniformity as belonging to category A, whereas those 
Chapter 2: Conceptual Category Learning (I)26

above it are classified as B. For the right-only condition, by contrast, there is considerably less evidence that people applied the descending partial boundary.

Insert Figure 2.3 about here

Extent of partitioning. The single-context conditions also afford an assessment of the extent of partitioning, by examining whether participants used a partial strategy in its appropriate test context as if the other one had never been learned.

I thus computed correlations between the classification probabilities of each test item in the systematic-context condition and the two single-context conditions. One correlation was computed within the same context (i.e., responses in the "left" context in the systematiccontext condition were correlated with responses in the left-only condition, and equivalently for the "right" context). The other correlation was computed across the two contexts (i.e., responses in the "left" context in the systematic-context condition were correlated with responses in the right-only condition, and equivalently for the "right" context). Note that both correlations were computed between subjects. The correlation was $\underline{r}=0.892(\underline{N}=20$; regression intercept and slope .04 and .94 , respectively, with mean absolute deviation .087) when contexts were the same and $\underline{r}=0.19$ (intercept and slope .38 and .20 , respectively, and mean absolute deviation .21) when contexts differed.

The magnitudes of these correlations are best interpreted in light of the correlation between contexts within the systematic-context condition. That correlation, which involved responses by the same participants across contexts, was $\underline{r}=0.55$ (intercept and slope -.08 and 1.05, respectively, mean absolute deviation .21), and hence considerably smaller than the between-condition correlation within the same context—even though the latter involved responses from different individuals. It follows that in the systematic-context condition, people in each test context classified transfer items in virtually the same way as people in the corresponding single-context condition who had never been exposed to the other context during training. 
Individual differences. I conclude by considering the individual differences in the systematic-context condition. The horizontal-boundary cluster used in Experiment 1 was omitted from this analysis because with the revised set of transfer items, this strategy was not distinguishable from use of the true boundary. Hence only three possible strategies were predefined for the $\underline{k}$-means cluster analysis, corresponding to application of (1) the correct bilinear boundary, (2) the ascending partial boundary, and (3) its descending counterpart. Table 2. $\equiv$ ows the resulting classification of participants in both test contexts. Several comments can be made about the classification: First, some $20 \%$ of participants seemed to apply the correct bilinear boundary in each context, whereas roughly half were identified as applying the appropriate partial boundary. I therefore conclude $\equiv$ the magnitude of the partitioning effect was greater than that observed in Experiment 1.

Insert Table 2.2 about here

Statistical support for the effect of test context on classification was provided by computing Cramer's coefficient $(\phi)$ for the association between test context and cluster membership in each condition. Cramer's coefficient is a transformation of $\chi^{2}$ and is readily interpretable as a measure of association that ranges from 0 to unity (Wickens, 1989). If people change their categorization strategy with test context, there should be a strong association between context and cluster membership. This expectation was confirmed by the magnitude of Cramer's coefficient $(\phi=.407, \underline{\mathrm{p}}<.02){ }^{1}$

Summary. This experiment gave rise to some notable results. First, in comparison to Experiment 1, I observed a much clearer indication that knowledge partitioning was present in a significant proportion of participants. Second, by examining the two single-context conditions, I confirmed an asymmetry of the partial boundaries that was independent of the context manipulation, and that probably reflected basic characteristics of category learning.

\footnotetext{
${ }^{1}$ Although Wickens (1989) suggests that repeated observations on the same participants may well be considered independent, a more conservative approach is to divide the underlying value of $\chi^{2}$ by 2 , thus accounting for repetition of individuals across contexts (Wickens, 1989). Applying the more conservative $\chi^{2} / 2$ yields a non-significant value of Cramer's coefficient $(\phi=.287, \mathrm{p}=.163)$.
} 
Chapter 2: Conceptual Category Learning (I)28

Third, the correlational analyses supported the conclusion that people in Experiment 2 used different context-gated components of knowledge independently. That is, in contrast to the findings by Aha and Goldstone (1992), in which people were shown to be sensitive to the existence of the other cluster when they generalized their responses along one of the boundaries, the present experiment revealed a more extreme case in which people generalized their responses along one of the boundaries as if they had never known the other. This confirms and extends the previous results in function learning reported by Lewandowsky et al. (2002). 


\section{Chapter 3: Conceptual Category Learning (II)}

The results thus far have supported expectations: Knowledge partitioning arises in category learning in a significant proportion of participants and, when it arises, it can be nearly complete. However, the category structure used thus far entailed two limitations. First, the difficulty of learning differed between the partial boundaries, which may have prevented knowledge partitioning if people were unable to use one of the partial boundaries. Second, the context manipulation was perfectly correlated with one of the predictors: In the systematic-context condition, all values of $\mathrm{X}$ below 400 were accompanied with one level of context, and those above 400 with the other. It follows that people may have partitioned their knowledge not only on the basis of context but also on the basis of the value of $\mathrm{X}$-indeed, for this reason the stimulus structure afforded the opportunity for partitioning even in the randomized-context condition. This, in turn, may have reduced the apparent size of the partitioning effect in the first two experiments.

\section{Experiment 3}

Experiment 3 used a new category structure that circumvented these problems. The category structure, shown in Figure 3. Ecluded two parallel ascending boundaries, with one category located in between the boundaries and the other one outside. As before, this implied that the set of learning instances could be divided into two clusters, each separated into two categories by its own local boundary. As in the first two experiments, context in the systematiccontext condition identified the two clusters and, by implication, a component boundary of the category space. However, unlike the earlier studies, both component boundaries were ascending and context was not perfectly correlated with either of the two predictors. To verify that participants could learn an integrated representation of this category structure, Experiment 3 again included a randomized-context condition. 
Insert Figure 3.0 about here

\section{$\underline{\text { Method }}$}

\section{$\underline{\text { Participants }}$}

Forty-eight undergraduate volunteers from the University of Western Australia participated voluntarily in exchange for partial course credit. An equal number of participants was randomly assigned to the systematic- and the randomized-context condition.

\section{$\underline{\text { Stimuli }}$}

The boundaries in Figure 3.0 are described by $|\mathrm{Y}-\mathrm{X}-100|=200$. As in the first two experiments, the category task was instantiated as two hypothetical species of tropical fish that had to be classified on the basis of "density” (X), “depth” (Y), and "season” (context).

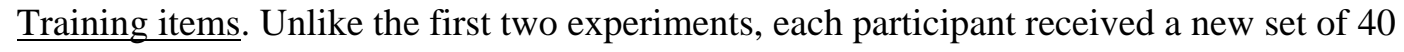
unique training instances that were randomly sampled from within the dotted rectangle in Figure 3.0 (not including locations in the immediate vicinity of the positive diagonal or the two boundaries). The figure includes a representative sample of items, with open circles and crosses denoting membership of category A and B, respectively. The set of 40 items was divided into two clusters of 20 stimuli located above (the "upper” cluster) and below (“lower”) the principal diagonal.

In the randomized-context condition, context cues were randomly assigned to training stimuli, subject to the constraint that each context label occurred with an equal number of items from both categories and both clusters. In the systematic-context condition, as in the preceding experiments, context did not predict category membership but predicted the cluster to which a training item belonged. That is, for a given participant, one level of context always occurred with stimuli within the upper cluster, and the other with those in the lower cluster. Assignment of 
context labels to cluster was counterbalanced and context is referred to generically as "upper" and "lower."

Regardless of condition, training consisted of 8 blocks of trials, each involving a different random sequence of the 40 items.

Transfer items. All participants were tested with the same set of 40 novel transfer stimuli, represented by filled diamonds in Figure 3.0. As in the first two experiments, there were two blocks of transfer trials, each with a different random order of stimuli. Each context appeared equally often in each block, and context alternated between blocks for each item.

Diagnosticity of transfer items. Transfer items came from three distinct areas in the category space, identified by number in Figure3.0. As before, the effect of context was expected to differ between areas depending on whether or not knowledge is partitioned. If partitioning is absent, as would be expected in the randomized-context condition, then context should have no effect in any of the areas, and items in Area 2 should be classified as belonging to category A, whereas those in Areas 1 and 3 should be classified as B.

The presence of partitioning would be indicated by a context-invariant tendency to classify items in Area 2 as belonging to category A, accompanied by a strong context effect in Areas 1

and 3. Specifically, in the upper test context, items in Area 1 should be classified as belonging to $\mathrm{B}$ and those in Area 3 as belonging to category A. Conversely, in the lower context, items in Area 1 should be classified as A and those in Area 3 as B.

\section{Procedure}

Details of the procedure, including presentation parameters and response keys, were the same as in the first two studies.

\section{$\underline{\text { Results and Discussion }}$}

\section{Training Performance}

The training data mirrored the outcome of the first two experiments. Performance improved from .564 (Block 1) to .752 (Block 8) across training, and participants performed better in the systematic-context condition $(\underline{M}=.717)$ than in the randomized-context condition $(\underline{M}=.652)$. 
Accordingly, a 2 (Condition) $\times 8$ (Block) between-within ANOVA revealed a main effect of Block $[\underline{F}(7,322)=26.981, \operatorname{MSE}=0.008, \underline{p}<.01]$, a marginal effect of Condition $[\underline{F}(1,46)=$ 3.752, $\mathrm{MSE}=0.105, \underline{\mathrm{p}}=0.059]$, and no interaction between these two variables, $\underline{\mathrm{F}}(7,322)<1$.

\section{Transfer Performance}

Knowledge partitioning. Table 3. $\equiv$ ggests that, as expected, test context had no effect on classification probabilities in any area in the randomized-context condition. In the systematiccontext condition, there was no effect of context in Area 2 (the middle region within the two component boundaries), whereas there were strong—and opposite—effects of context in Area 1 and Area 3. This pattern conformed to what would be expected under knowledge partitioning.

Insert Table 3.0 about here

The corresponding 2 (Condition) $\times 2$ (Test context) $\times 3$ (Area) between-within ANOVA revealed a highly significant main effect of $\operatorname{Area}[\underline{F}(2,92)=107.5, \operatorname{MSE}=0.06, \underline{p}<.01]$. In addition, the main effect of Condition approached significance with $\underline{\mathrm{F}}(1,46)=3.37$, MSE $=$ $0.11, \underline{p}=.073$, whereas there was no discernible effect of Test context $[\mathrm{F}(1,46)<1]$. Two of the three two-way interactions were also significant: Condition $\times$ Area $[\underline{F}(2,92)=4.3, \operatorname{MSE}=0.06$, $\mathrm{p}<.05]$ and Test Context $\times$ Area $[\underline{\mathrm{F}}(2,92)=11.97, \mathrm{MSE}=0.05, \underline{\mathrm{p}}<.01]$.

Finally, the over-arching three-way interaction involving all experimental variables was significant $[\underline{F}(2,92)=12.16$, MSE $=0.05, \underline{p}<.001]$ and was further explored by separate twoway ANOVAs in each condition. In the randomized-context condition, there was no Test context $\times$ Area interaction $[\underline{F}(2,46)<1]$, whereas in the systematic-context condition that interaction was highly significant $[\underline{F}(2,46)=18.27, \operatorname{MSE}=0.07, \underline{p}<.001]$. As suggested by the table, the Test context $\times$ Area interaction in that condition involved significant effects of context in Areas 1 and $3[\underline{\mathrm{F}}(1,23)=13.62, \operatorname{MSE}=0.07, \underline{\mathrm{p}}<0.01$, and $\underline{\mathrm{F}}(1,23)=21.55, \mathrm{MSE}=0.07, \underline{\mathrm{p}}<.001$, respectively] but not Area $2[\underline{\mathrm{F}}(1,23)<1]$. 
In extension of the first two experiments, this suggests that participants applied different partial knowledge in different contexts. One critical difference to the earlier studies was the apparent symmetry of the effect: The size of the context effect was virtually identical for Areas 1 and 3, presumably because both partial boundaries were ascending and thus did not differ in difficulty.

However, as in the earlier studies, the absolute magnitude of the effect warranted further exploration. Specifically, if people used context-appropriate partial boundaries, then items in Areas 1 and 3 should be consistently classified as belonging to category A in the lower and upper contexts, respectively. Indeed, given their greater distance from the boundary, items in those areas should be even more uniformly classified as A than items in the central area, some of which may on occasion be considered to lie on the other side of a partial boundary. In actual fact, however, the probability of classification was near 0.5 in both areas. Similar to the earlier experiments, this could have either reflected chance performance or the fact that some individuals partitioned their knowledge whereas others did not. These alternatives were teased apart by the individual-differences analysis.

Individual differences. Using three pre-defined clusters consisting of (1) the correct parallel boundaries, (2) the upper partial boundary, and (3) the lower partial boundary, individual response profiles were entered into a $\underline{\mathrm{k}}$-means cluster analysis for each condition and test context separately. Several comments apply to the results which are shown in Table 3.

Insert Table 3.1 about here

First, in contrast to Experiments 1 and 2 (see Tables 2.2 and 2.3) there were very few cases (only 3 people) that escaped identification as using one of the three strategies. This further confirmed that the current category structure eliminated some of the problems associated with the earlier stimuli. Second, in the randomized-context condition, almost nobody used partial boundaries, whereas a considerable proportion of participants uniformly applied the true 
boundary across both contexts. The negligible value of Cramer's coefficient $(\phi=.059)$ supports the conclusion that in this condition people's choice of strategy was unaffected by test context. In the systematic-context condition, by contrast, between a third (lower context) and half (upper) of all participants applied the context-appropriate partial boundary. The crucial role of context was affirmed by a highly significant Cramer's coefficient $(\phi=.677, \underline{\mathrm{p}}<.001)$. Even with the conservative correction $\left(\chi^{2} / 2\right)$, the association between context and cluster remained significant $(\phi=.479, \underline{\mathrm{p}}<.012)$

To further illustrate the response pattern associated with use of the true and partial boundaries, Table 3.0 also shows the data for two sub-groups of participants in the systematiccontext condition. The panel labeled true-boundary shows the classification probabilities from 11 participants who uniformly applied the true boundary in both contexts, whereas the knowledge partitioning panel shows the data of 8 participants who showed complete partitioning by using the appropriate partial boundary in each of the two contexts. This confirms that individuals who were identified as having partitioned their knowledge by a fairly loose criterion — exhibiting a generalization profile that was more similar to that expected under partitioning than to the true boundary_completely reversed their categorization judgment between contexts in Areas 1 and 3. Conversely, there was no effect of context in Areas 1 and 3 for those people who were identified as using the true boundary by an equally loose criterion. It follows that the overall classification probabilities arose from a probability mix of two distinct groups of subjects, rather than uniform chance performance.

\section{Experiment 4}

In all experiments thus far, context did not directly predict classification. Nonetheless, context was uniformly found to gate performance, at least in a significant proportion of participants, when it predicted which partial boundary to apply to the task. The fourth and final experiment examined whether context would be used to predict classification by adding a contingency rating task (e.g., Wasserman \& Berglan, 1998; Williams, Sagness, \& McPhee, 
1994). The contingency rating task, presented after the conventional transfer test, asked people to provide a numeric estimate of the contingency between values of each predictor by itself and the outcome.

Experiment 4 included only the systematic-context condition, which was nearly identical to that of Experiment 3, except that the transfer test included several training items in addition to the usual novel stimuli.

\section{$\underline{\text { Method }}$}

\section{$\underline{\text { Participants }}$}

Twenty-four undergraduate volunteers from the University of Western Australia participated in exchange for partial course credit.

$\underline{\text { Stimuli }}$

The category structure and stimuli were the same as in Experiment 3. The transfer test differed by additionally including 20 randomly chosen training instances ( 5 items from each category in each cluster).

In the final contingency rating task, one predictor was shown at a time, and ratings were obtained for 4 possible values of X (“density” 200, 400, 600 and 800), 4 possible values of Y (“depth” 100, 300, 500 and 700), and the two possible levels of context (“summer” and “winter”). Owing to a software error, some predictor values were repeated and for a small number of participants some values of $\mathrm{Y}$ were omitted.

\section{$\underline{\text { Procedure }}$}

The procedure was the same as in Experiment 3, except that the contingency test followed the transfer test. On each contingency rating trial, participants used the right- and left-arrow keys to move a vertical bar on the screen along a horizontal scale to indicate their rating.

The scale was marked by a category label at each end, with the assignment of labels counterbalanced across participants. Each end of the scale represented 100\% certainty that an item with the given predictor value would belong to that category. 


\section{$\underline{\text { Results and Discussion }}$}

\section{Training Performance}

Performance improved across the 8 training blocks from .547 (Block 1) to .8 (Block 8), which was confirmed to be significant by a one-way within-subjects ANOVA $[\underline{F}(7,161)=$ 23.452, $\mathrm{MSE}=0.008, \mathrm{p}<.01]$. In absolute terms, performance was nearly indistinguishable from the systematic-context condition in Experiment 3.

\section{Transfer Performance}

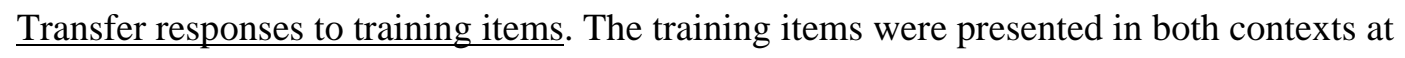
test. One of those (called "congruent”) occurred at training, whereas the other one was novel for the particular conjunction of X and Y values (“incongruent”). Strictly speaking, incongruent items were thus no longer old exemplars.

Classification probabilities for training items are shown by context and area in Table 3 the congruent context, responses reflected knowledge of the true boundary, with items in the central Area 2 being predominantly classified as A and those in the exterior Areas 1 and 3 as B. In the incongruent context, by contrast, there was no evidence that people used the correct boundary. Categorization probabilities reversed for the two exterior areas, giving rise to an effect of Context in Area $1[\underline{F}(1,23)=15.4, \operatorname{MSE}=0.08, \underline{p}<.01]$ and in Area $3[\underline{F}(1,23)=26.74$, $\operatorname{MSE}=0.09, \mathrm{p}<.01]$

Insert Table 3.2 about here

Transfer responses to novel test items. Table 3. 三ows that responses to the novel transfer items mirrored the results of the previous experiment. The relevant 2 (Test context) $\times 3$ (Area) within-subjects ANOVA revealed a significant main effect of Area $[\underline{F}(2,46)=33.05$, MSE $=$ $0.08, \underline{\mathrm{p}}<.001]$ and a significant interaction between both variables $[\underline{\mathrm{F}}(2,46)=27.34, \mathrm{MSE}=$ 0.05, $\mathrm{p}<.001] . \equiv$ follow-up simple comparisons between test contexts were significant in 
Area $1[\underline{F}(1,23)=12.53, \operatorname{MSE}=0.05, \underline{p}<.01]$ and in Area $3[\underline{F}(1,23)=28.26$, MSE $=0.08, \underline{p}<$ $.001]$ but not in Area $2[\underline{F}(1,23)<1]$.

\section{Insert Table 3.3 about here}

The $\underline{\mathrm{k}}$-means cluster analysis showed that about half of the participants $(\underline{\mathrm{N}}=10)$ applied the true boundary in both contexts, whereas about a third of the participants $(\underline{\mathrm{N}}=7)$ showed complete and symmetric partitioning by using the upper boundary in the upper context and the lower boundary in the lower context. Cramer's coefficient $(\phi=.676, \underline{\mathrm{p}}<.001)$ confirmed that context affected strategy choice. The effect persisted even with the conservative $\left(\chi^{2} / 2\right)$ adjustment; $\phi=$ $.478, \mathrm{p}<.012$. The transfer responses to novel items for those two subgroups of participants are shown in the corresponding panels of Table 3.3.

Comparison of novel and training items. The two classes of items were compared by computing the differences between upper and lower test contexts (not between congruent and incongruent) ${ }^{1}$ for each item class and each area. Those differences were entered into a 2 (Item type; training vs. novel) $\times 3$ (Area) within-subjects ANOVA. The only significant effect was a main effect of Area with $\underline{F}(2,46)=32.14, \operatorname{MSE}=0.19, \underline{p}<.01$. Neither the effect of item type nor the interaction between both variables was significant (both $\underline{F}$ 's $<1$ ). This finding implies that people considered training items presented in a new context at test as being completely novel and indistinguishable from a new pairing of values for $\mathrm{X}$ and $\mathrm{Y}$.

An effect of memory for training instances was only observed when training items were presented in the same context at test: As shown in the earlier Table 3.2, it was only under those narrowly defined circumstances that classifications were compatible with the true bilinear category boundary.

\section{Contingency Ratings}

Contingency ratings were analyzed by expressing responses as the judged probability of an item belonging to category A. Table 3. $\equiv$ ows the average and two-subgroups ratings for all 
tested levels of the three predictors (X, Y, and context) together with the normative values (computed for the overall set of training items). Responses are shown averaged across all participants and for the two major subgroups identified by the $\underline{\mathrm{k}}$-means analysis separately.

\section{Insert Table 3.5 about here}

In order to determine whether judged contingencies departed from chance, the mean response at each level of each predictor was compared to .5 by a single-sample t-test. Using a significance level of .05 for each test, three predictor levels were found to depart from chance; those are identified by asterisks in the table. With a more stringent Bonferroni adjustment to significance, none of the 10 responses differed from chance.

Thus, there was some suggestion that people were sensitive to the role of predictors $\mathrm{X}$ and Y, but their judged contingencies clustered relatively close to chance and were not nearly as extreme as the normative values. There was little evidence that the two groups of participants differed from each other. For the context variable, there was no evidence that people erroneously judged it to be predictive, as their responses were close to the normatively correct chance value (largest $\underline{\mathrm{t}} \approx 1.00$ ) and also did not differ between the two contexts $[\mathrm{t}(23)<1$, using all participants]. The judged contingency of context also did not differ between groups of participants. This result was also confirmed by participants in either the knowledge partitioning or true-boundary group.

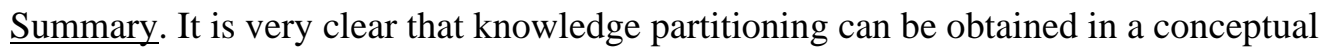
category learning paradigm. The first four experiments all showed that people can learn to use context to gate use of partial categorization knowledge. In Experiments 3 and 4, the usage of partial knowledge was completely symmetrical. Experiment 2 additionally showed that when people partitioned their knowledge, they used each partial knowledge component as though the other one had not been acquired. That is, performance in each context was very similar to the performance of people in comparison groups who had only been trained on half of the stimulus 
space in that one context, suggesting that the partitioned components of knowledge were largely independent of each other. Finally, Experiment 4 confirmed that people were at least somewhat sensitive to the normative role of each predictor in isolation, including the fact that context by itself did not predict category membership.

Another consistent outcome was that training performance was better whenever knowledge partitioning was observed at transfer. Thus, in Experiments 1 and 3 performance in the systematic-context condition was significantly better than in the randomized-context condition.

The pattern of training and transfer performance parallels the related findings reported by Lewandowsky et al. (2002) in a function learning paradigm. This reinforces the close empirical and conceptual connection between those two modes of concept learning and extends the generality of the knowledge partitioning framework. However, before considering the wider implications of our results, several limitations must be acknowledged.

\section{Limitations of the First Four Experiments}

The first limitation concerns the magnitude of the knowledge partitioning effect. Across experiments, the proportion of participants who partitioned their knowledge when given the opportunity to do so ranged from $20 \%$ to at most $50 \%$. A significant proportion of the remaining participants applied the correct complex boundary, while still others were not clearly identifiable as using one or the other strategy. This suggests that knowledge partitioning is but one of several ways in which people may choose to master a complex categorization task.

A second potential limitation concerns the use of pseudo-continuous predictors involving numeric labels. Although this is not without precedent in categorization research (e.g., Erickson \& Kruschke, 1998; Lewandowsky et al., 2000), the practice has been criticized by Nosofsky and Johansen (2000). However, the two experiments in the next chapter provide a solid support for knowledge partitioning in categorization with perceptual stimuli similar to those used by Nosofsky and Johansen (2000). 


\section{Chapter 4: Perceptual Category Learning}

Contrary to the conventional and widespread assumption that people's representations are homogeneous and integrated, the first four experiments showed that people often master a complex categorization task by forming largely independent components or "parcels" of knowledge. It was also shown that once a knowledge parcel is chosen, it provides the sole basis of performance, to the exclusion of knowledge demonstrably present in other parcels. These results support and extend related findings in the areas of expertise (Lewandowsky et al., 2000), and function learning (Kalish et al., in press; Lewandowsky et al., 2002).

One limitation of the first four studies comes from the case of stimuli involving numeric labels. The numeric labels indicated the precise magnitude along each stimulus dimension, thus participants could rely on these numerical values, even do some calculations on them, to generate rules. This might be an explanation for the function-learning phenomenon occurring in a category-learning task. The influence of the numeric labels on category learning was also found in the Erickson and Kruschke (1998) study. In this study, since numeric tic-marks were used as referents for the dimensional magnitudes of perceptual stimuli, some participants were able to apply a rule which involved the numeric labels to those exception stimuli (see Nosofsky \& Johansen, 2000, footnote 3). Thus, the category representation for the items might correspond to not only the stimulus dimensions experimenters manipulated, but also the numeric values the participant is exposed to (Nosofsky \& Johansen, 2000). Consequently, the relationships between items in the psychological space might be altered, given that the numeric tick-marks were possibly used as dimensions in the psychological space which the participants perceived.

Following this issue, two experiments extended the work of the preceding studies to a perceptual category learning task that did not use any numeric labels. Should partitioning be obtained under these circumstances, it would underscore the generality of the knowledge partitioning framework, and would provide a benchmark set of results for the evaluation of potential computational models. 


\section{Experiment 5}

This experiment used the same category structure as Experiment 4 shown earlier in Figure 3.1. Unlike Experiment 4, the stimuli were instantiated using graphical elements as in the study

conducted by Erickson and Kruschke (1998, 2001, 2002). Figure 4. $\equiv$ ows two example stimuli composed of a rectangle and a vertical line segment within it. Stimuli varied along two dimensions, the position of the line segment (X) and the height of the rectangle (Y). The context cue was instantiated by the color (i.e., red or green) of the rectangle and line segment.

The experiment, like Experiments 1 and 3, included two conditions: In the randomizedcontext condition, context cues were randomly assigned to an equal number of training instances from each category and each cluster. In the systematic-context condition, by contrast, context was consistently mapped to clusters. Thus, context on its own did not predict category membership (because each cluster contained an equal number of items from each category), but did identify whether a stimulus was close to the upper or lower partial boundary.

Insert Figure 4.0 about here

\section{Method}

\section{$\underline{\text { Participants }}$}

Forty-eight undergraduate volunteers from the University of Western Australia participated in exchange for partial course credit. An equal number of participants was randomly assigned to each condition.

\section{$\underline{\text { Stimuli }}$}

The two categories were arbitrarily instantiated as different kinds of "space shuttle", and the dimensions $\mathrm{X}$ and $\mathrm{Y}$ were, respectively, the height of the rectangle and the segment position within it. The dimensional values shown in Figure 3.1 were rescaled to map into the physical 
extent of the stimulus display. Additionally, stimuli were differentiated by color (thus instantiating context) and were presented in either red or green.

Training items. Each participant received a unique set of 40 training items that were randomly sampled from within the dotted rectangle in Figure 3.1. The figure includes a representative sample of items, with open circles and crosses denoting membership in category A and B, respectively. The set of 40 training items was divided into two clusters of 20 stimuli located above (“upper” cluster) and below (“lower”) the positive diagonal.

In the randomized-context condition, the context dimension (i.e., color) was randomly assigned to training stimuli with the constraint that, in each cluster, there were equal numbers of training stimuli in each category presented in the two contexts.

In the systematic-context condition, context predicted the cluster to which a training item belonged, without however predicting category membership. Thus, stimuli within the upper cluster were all presented in one color, and those within the lower cluster in the other color, with an equal number of items in each color belonging to each category. Assignment of color to cluster was counterbalanced across participants.

In both conditions there were 8 blocks of training trials, each involving a different random sequence of the 40 items.

Transfer items. There were 60 transfer items in total. Of those, 40 novel items were the same for all participants and are represented by filled diamonds in Figure 3.1. The remaining 20 items were randomly chosen training instances (5 items from each category in each cluster). The sequence of 60 transfer items was presented twice, in a different random order within each sequence and with context alternating between sequences.

Diagnosticity of transfer items. Since the category structure is the same as used in Experiment 4, the diagnostic transfer items are still in Area 1 and 3 (see Figure 3.1). If partitioning is present, then the prediction to the item in these two areas will be reversed when the test context reverses. If partitioning is absent, namely, true-boundary is learned, then the prediction to the item in these two areas will not change when the test context changes. 


\section{$\underline{\text { Procedure }}$}

Participants were tested individually in a quiet booth. Each trial commenced with display of a fixation signal (a “+”) in the center of the screen for $500 \mathrm{~ms}$, followed by presentation of the stimulus which remained visible until participants responded. The width of rectangle was kept constant as $12.5 \mathrm{~cm}$ for all stimuli and the height of rectangle varied from $2.8 \mathrm{~cm}$ to $13.2 \mathrm{~cm}$. The distance of the segment position to the left margin of rectangle varied from $1 \mathrm{~cm}$ to $11 \mathrm{~cm}$. All lines were $0.2 \mathrm{~cm}$ wide and colored red or green as dictated by context.

In the training phase, participants were given written feedback (the word "correct" or "wrong” shown in the center of the screen) for $1000 \mathrm{~ms}$ after each response, before the next trial commenced. No feedback was presented during transfer.

Self-paced breaks were inserted after every 40 training trials and after every 60 transfer trials. Additionally, there was a break between the training and transfer phases. The experiment lasted just under one hour.

\section{$\underline{\text { Results and Discussion }}$}

\section{Training Performance}

Performance improved considerably with training for both conditions, from .53 (Block 1) to .77 (Block 8). Participants performed slightly better in the systematic-context condition $(\underline{\mathrm{M}}=.70)$ than in the randomized-context condition $(\underline{\mathrm{M}}=.66)$. Accordingly, a 2 (Condition) $\times 8$ (Block) between-within ANOVA revealed a main effect of Block, $\underline{\mathrm{F}}(7,322)=42.21, \mathrm{MSE}=0.007, \mathrm{p}<$ .01 , no main effect of Condition, $\underline{F}(1,46)=2.18$, MSE $=0.08, \underline{p}<.15$, and no interaction between those two variables, $\underline{\mathrm{F}}(7,322)<1$.

The training results confirm that people can learn the parallel category boundaries. Although people in the systematic-context condition performed slightly better than people in the randomized-context condition, the difference (unlike in the conceptual category-learning task of Experiment 3 and 4) was not statistically significant.

\section{Transfer Performance}


Performance on the 20 training items shown at transfer was analyzed first separately from performance on the 40 novel items.

Transfer responses to training items. Training items were tested in both contexts. One of those (which I call “congruent”) occurred at training, whereas the other one had not been present at training with that particular conjunction of $\mathrm{X}$ and $\mathrm{Y}$ values (“incongruent”).

Classification probabilities for training items are shown by context and area in Table 4. with the randomized-context and systematic-context conditions in the top and bottom panel, respectively. In the randomized-context condition, there is evidence of consistent application of the parallel boundaries, with items in Area 2 being predominantly classified as A and those in Area 1 and Area 3 as B, irrespective of whether test and training contexts matched. In confirmation, none of the simple comparisons between contexts were significant in this condition.

Insert Table 4.0 about here

In the systematic-context condition, by contrast, the parallel boundaries were applied only when the test context was congruent with the training context. When the test context was incongruent, people responded to the "training” items quite differently and there was little evidence that the parallel boundaries were applied. In confirmation, there was a marginally significant effect of context (congruent vs. incongruent) in Area 1, $\underline{F}(1,23)=3.89, \operatorname{MSE}=0.09$, $\underline{\mathrm{p}}=.061$, and a significant effect of context in Area 3, $\underline{\mathrm{F}}(1,23)=5.11$, MSE $=0.09, \underline{\mathrm{p}}<.05$. Overall, this suggests that people treated training items presented in a different context as though they were novel transfer stimuli. Recall that in Experiment 4, the predicted probabilities of Category A response on the training items in Area 1 and 3 were also increased in an incongruent context. These results consistently suggest that in the systematic-context condition, context would be taken into consideration for generating responses regardless of the type of categorylearning task. 
Novel transfer items. Transfer responses to novel items are shown in Table 4. expected, test context had no effect on classification probabilities in any area in the randomizedcontext condition. By contrast, context had a strong effect in Areas 1 and 3 in the systematiccontext condition.

Insert Table 4.1 about here

The transfer responses were explored further with a 2 (Condition) $\times 2$ (Test context) $\times 3$ (Area) between-within ANOVA. The main effect of area was significant, $\underline{F}(2,92)=48.08$, MSE $=0.07, \mathrm{p}<.01$, but there were no significant effects of condition, $\underline{\mathrm{F}}(1,46)=1.32$, or test context, $\underline{\mathrm{F}}(1,46)=1.16 . \equiv$ over-arching three-way interaction was significant with $\underline{\mathrm{F}}(2,92)=10.19$, MSE $=0.05, \mathrm{p}<.01$. The interaction was explored by separate two-way within-participants ANOVAs for each of the two conditions. In the randomized-context condition, there was no trace of an interaction between test context and area, $\mathrm{F}(2,46)<1$. That interaction was, however, highly significant in the systematic-context condition, $\underline{F}(2,46)=15.48 \mathrm{MSE}=0.06$, $\mathrm{p}$ $<.01$. The corresponding simple comparisons between test contexts were significant in Area 1, $\underline{F}(1,23)=12.44, \operatorname{MSE}=0.06, \underline{p}<.01$, Area $3, \underline{F}(1,23)=16.47, \operatorname{MSE}=0.07, \underline{p}<.01$, and in Area $2[\underline{\mathrm{F}}(1,23)=6.89, \mathrm{MSE}=0.01, \underline{\mathrm{p}}<.05]$.

As expected under knowledge partitioning, classification probabilities differed substantially_and in opposing directions_-between contexts in Area 1 and in Area 3. This suggests that participants divided the categorization space into two, each with a partial boundary that was applied on the basis of context. The significant context effect in Area 2 was unexpected; below, I show that this reflects asymmetrical learning of the two partial boundaries.

Individual differences. As with Experiment 3, the participants in this experiment were classified by $\underline{\mathrm{k}}$-means cluster analysis into three subgroups according to their individual categorization strategies. Table 4. $\equiv$ ows the assignment of participants to clusters for each condition and test context. In confirmation of the aggregate analysis, most participants in the 
randomized-context condition applied the parallel boundaries in both contexts. In the systematiccontext condition, by contrast, the distribution of categorization strategies differed greatly between contexts. Although there were still a considerable number of participants who applied the parallel boundaries in both contexts, $46 \%$ (11) of participants used the upper partial boundary in the upper context (but none used it in the lower context), and conversely, 50\% (12) of participants used the lower boundary in the lower context (but only 2 participants used it in the upper context). Statistical support for the association between test context and classification was provided by Cramer's coefficient $(\phi)$ which was highly significant $(\phi=.616, \mathrm{p}<.001) \equiv$

Insert Table 4.3 about here

For all subsequent analyses and the forthcoming model fitting, two subgroups of participants were formed with the data aggregated within each group. Participants were assigned to a "knowledge partitioning" group $(\underline{\mathrm{N}}=9)$ if the cluster analysis classified them into the upper rule cluster in the upper context and to the lower rule cluster in the lower context. If the cluster analysis assigned a participant to the true boundary cluster in both contexts, then he or she would be assigned to the "true-boundary" subgroup $(\underline{\mathrm{N}}=10)$.

$\equiv$ noteworthy aspect of the data concerns the unexpected effect of test context in Area 2 for the knowledge partitioning group (the effect of context on the classification probability found to be significant; $\underline{\mathrm{F}}(1,8)=7.15$, MSE $=0.02, \underline{\mathrm{p}}<.05)$, which is absent in the true-boundary group. This effect may reflect an asymmetry of the two partial boundaries applied by that subgroup.

Figure 4. $\equiv$ ows the classification probabilities for each novel transfer item for participants in the KP group. The top panel shows the upper test context and the bottom panel the lower context. Every plotted square represents a novel transfer item, and the degree of shading indicates the likelihood of the item being classified into category A. The figure reveals a distance effect in both contexts, with items further away from each partial boundary being classified with 
more extreme probabilities. There is also some apparent asymmetry between contexts, with the upper context resulting in a sharper delineation between response types than the lower context. I suggest that this asymmetry underlies the context effect in Area 2. Because it is of little theoretical interest I do not consider it further.

Insert Figure 4.1 about here

Comparison of novel and training items. The two classes of items were compared by computing the differences between upper and lower contexts for each item class and each area. ${ }^{1}$ In the randomized-context condition, those differences were entered into a 2 (Item type; training vs. novel) $\times 3$ (Area) within-participants ANOVA which did not yield any significant effects. The parallel analysis of the systematic-context condition, by contrast, yielded a significant main effect of area, $F(2,46)=10.52, \mathrm{MSE}=0.26, \mathrm{p}<.01$, but no other effects. The absence of any item type effects confirms that people did not differentiate between completely novel combinations of $\mathrm{X}$ and $\mathrm{Y}$ and training items presented in a novel context but with an old $\mathrm{X}-\mathrm{Y}$ pairing. This replicates a similar effect reported in Experiment 4.

\section{Experiment 6}

Experiment 5 provided further evidence for the existence of knowledge partitioning in categorization. This confirms that the earlier results of conceptual category learning were not tied to the use of numeric categorization stimuli. Experiment 5 also underscored the presence of large individual differences in the systematic-context condition, with two subgroups of participants using two very different representations or strategies to classify test items. Experiment 6 examined two additional attributes of knowledge partitioning using the same

\footnotetext{
${ }^{1}$ For training items presented at transfer, the "incongruent” context differed between areas. In Area 3, it was the "upper" context that was incongruent at test, because no item below the diagonal appeared in that context during training. Conversely, in Area 1, it was the "lower" test context that was incongruent. To simplify comparison with novel transfer stimuli, this analysis distinguished between upper and lower contexts irrespective of congruency.
} 
stimulus space as the preceding study. They are (1) whether participants used the context cue to predict directly any outcome and (2) whether the hypothesized "knowledge parcels" (i.e., representations of the partial boundaries) were independent.

The first concern will be dealt with by asking participants to rate the contingency between each dimension and category responses, as was done in Experiment 4. The second concern will be dealt with by comparing the participants' performance in the systematic-context condition with the ones in the two single-context conditions, same as did in Experiment 2. If the performance in the systematic-context condition is highly correlated with the ones in the singlecontext conditions, then the independence of knowledge parcels can be confirmed. Therefore, in this experiment, there were three conditions: one systematic-context condition and two singlecontext conditions and also the participants in the systematic-context condition were asked to do the contingency rating task after finishing the transfer phase.

Because Experiment 5 showed that people can learn the parallel boundaries when context is randomly assigned to training stimuli, the randomized-context condition was not included in this experiment.

\section{$\underline{\text { Method }}$}

\section{$\underline{\text { Participants }}$}

Forty-eight undergraduate volunteers from the University of Western Australia participated in exchange for partial course credit. An equal number of participants (16) was randomly assigned to each condition: the systematic-context condition, the upper-only context condition and the lower-only context condition.

\section{$\underline{\text { Stimuli and Procedure }}$}

Systematic-context condition. The category structure and stimuli were the same as in the previous experiment. The usual transfer phase was followed by the contingency rating task involving 10 trials. On each contingency trial, a single dimensional value was shown, and ratings were obtained for 4 possible values of X (“segment position” 200, 400, 600, and 800), 4 possible values of Y (“height” 100, 300, 500, and 700), and the two possible levels of context (“red” and 
“green”). For example, when the value of X (segment position) was to be rated, a white vertical segment was presented whose lower end touched a horizontal line equal in length to the rectangle in the complete stimulus. When the value of Y (height) was to be rated, a white rectangle was presented without a vertical segment. For context, the word "Red" or "Green” was presented in its corresponding color.

To make their contingency judgment, participants used the right- and left-arrow keys to move a vertical bar on the screen along a horizontal scale. The scale was marked by a category label at each end, with the assignment of labels counterbalanced across participants. Each end of the scale represented $100 \%$ certainty that an item with the given dimensional value would belong to that category.

Single-context conditions. In the upper-only condition, the 20 learning instances were all located in the upper half of the stimulus space and were presented with a single context cue (red or green, counterbalanced across participants). Conversely, training in the lower-only condition involved the other 20 learning instances. There were 8 blocks of training trials in both conditions. Each block contained a different random sequence of the training items. In the transfer phase, all 20 learning items were presented together with the 40 novel transfer items from the systematic-context condition.

The procedure in all conditions was identical to that of Experiment 5.

\section{$\underline{\text { Results and Discussion }}$}

\section{Training}

Performance improved with training from .63 (Block 1) to .83 (Block 8). A 3 (Condition) $\times$ 8 (Block) between-within participants ANOVA confirmed the presence of a significant effect of block, $\underline{F}(7,315)=24.827, \operatorname{MSE}=0.009, \underline{p}<.01$, but found no effect of condition and no interaction between the two variables, F's $\leq 1$.

The absence of condition effect is noteworthy. First, both boundaries were equally easy to learn, given that there is no difference in learning the two single-context conditions. Second, 
knowledge of each boundary was captured without any lose by participants in the systematiccontext condition.

\section{$\underline{\text { Transfer }}$}

Transfer responses to training items. Table 4. areas, and test contexts, again using "congruent" and "incongruent” to refer to context being the same or different, respectively, between training and test. In the systematic-context condition, a 2 (Test context; congruent vs. incongruent) $\times 3$ (Area) within-participants ANOVA revealed a significant interaction between both variables, $\underline{\mathrm{F}}(2,30)=9.28, \mathrm{MSE}=0.04, \underline{\mathrm{p}}<.01$. Further exploration by simple comparisons revealed that the effect of context was significant in Areas 1 and 3, $\underline{\mathrm{F}}(1,15)=8.35$, MSE $=0.13, \underline{\mathrm{p}}<.05$ and $\underline{\mathrm{F}}(1,15)=8.59, \mathrm{MSE}=0.02, \underline{\mathrm{p}}<.05$, respectively, but not in Area $2, \underline{\mathrm{F}}<1$. Thus, as in the previous experiment, the effect of context in Areas 1 and 3 suggests that training items were regarded as novel when presented in an incongruent context.

Insert Table 4.3 about here

The same analyses were applied to the two single-context conditions, except that the area variable had two levels only (because training items were drawn only from Areas 1 and 2 in the upper-only condition and Areas 2 and 3 in the lower-only condition). In both conditions, only the effect of area was significant, with $\underline{\mathrm{F}}(1,15)=36.62$, MSE $=0.09, \underline{\mathrm{p}}<.01$ and $\underline{\mathrm{F}}(1,15)=$ 52.42, MSE $=0.06, \underline{\mathrm{p}}<.01$, for upper- and lower-only, respectively. The effect of context was not significant, with the remaining $\underline{F}$ 's $\leq 1.79$ and $\underline{\text { ' }}$ s $\leq 2.08$ in the upper- and lower-only conditions, respectively. Thus, it is clear that people completely ignored context in the singlecontext conditions. Given that context was invariant during training, thus limiting its role to an unexplained change in color of the stimuli at transfer, the result does not come as a surprise.

Transfer responses to novel items. Table 4. $\equiv$ ows the classification responses to novel transfer items for all areas and conditions. As in Experiment 5, the pattern for the systematic- 
context condition was suggestive of knowledge partitioning. This was confirmed by the 2 (Test context) $\times 3$ (Area) within-participants ANOVA for that condition, which revealed a significant interaction between context and area, $\underline{F}(2,30)=8.17, \mathrm{MSE}=0.08, \underline{\mathrm{p}}<.01$. Exploration of the interaction by simple comparisons between contexts revealed significant effects in Areas 1 and $3, \underline{\mathrm{F}}(1,15)=8.63, \mathrm{MSE}=0.09, \underline{\mathrm{p}}<.05$ and $\underline{\mathrm{F}}(1,15)=7.13, \mathrm{MSE}=0.08, \underline{\mathrm{p}}<.05$, respectively, but not in Area 2, $\underline{\mathrm{F}}<1$.

Insert Table 4.4 about here

A $\underline{k}$-means cluster analysis paralleling that of Experiment 5 was conducted, with the results shown in Table 4.3. The table shows that performance was quite dependent on context, with $50 \%$ (8) of participants applying the upper boundary in the upper context and $43.75 \%$ (7) of participants applying the lower boundary in the lower context. A significant Cramer's coefficient $(\phi=.624, \mathrm{p}<.01$; conservative correction $\phi=.441, \mathrm{p}<.012)$ confirmed the strong association between cluster and context. This replicated the knowledge-partitioning effect of the previous experiment. For the subsequent analyses, participants were assigned to the knowledge partitioning $(\underline{\mathrm{N}}=5)$ and the true-boundary $(\underline{\mathrm{N}}=5)$ groups in the same manner as in Experiment 5.

Turning to the single-context conditions, Table 4.4 suggests that participants learned the partial boundary appropriate to each condition, although it was applied less rigorously in the incongruent test context (i.e., the upper context for the lower-only condition and vice versa). Specifically, the differences between the extreme areas (1 and 3) were greater when the test context matched the one encountered during training. In confirmation, combined analysis of the single-context conditions in a 2 (Condition) $\times 2$ (Test context) $\times 3$ (Area) between-within participants ANOVA revealed a significant interaction involving all three variables, $\underline{\mathrm{F}}(2,60)=$ 6.24, $\mathrm{MSE}=0.04, \underline{\mathrm{p}}<.01$. 
To sum up, the fact that performance on novel transfer items differed between singlecontext conditions in the diagnostic regions (Areas 1 and 3), but not in the central area (Area 2) for which both boundaries predict the same response, shows that participants learned different linear partial boundaries in the two conditions. Moreover, participants applied the partial boundary less stridently to novel transfer items in the incongruent context, which replicates a similar observation in Experiment 2.

\section{Modularization of classification rules}

One aim of this experiment was to examine the extent to which knowledge "parcels" became modularized and encapsulated during training. To address this issue, item-wise correlations were computed both within and across conditions (i.e., for each transfer stimulus, the proportion of category A responses was computed across participants for each context). First, a between-context correlation was computed for all participants in the systematic-context condition. This provides a measure of the extent to which knowledge is integrated across the two contexts. The relatively low value of the correlation $(\underline{r}=.326)$ suggests that people responded to items quite differently in the two contexts.

Second, a within-context correlation was computed between each single-context condition and responses made to the same items (but by different participants) in the corresponding context in the systematic-context condition. This within-context correlation $(\underline{r}=.677)$, despite being computed between individuals, was higher than the correlation between contexts computed within the same participants. When the within-context correlation was computed including only the knowledge partitioning group from the systematic-context condition, the correlation increased considerably to $\underline{r}=.896$. The high within-context correlation implies that participants who partitioned their knowledge applied each partial boundary without any consideration of the other one-their behavior in each context was highly similar to that of people who only ever learned one boundary. This conclusion is further supported by a negative between-context correlation for the knowledge partitioning group $(\underline{r}=-.738)$ which reflects the fact that the two partial boundaries dictate opposite classifications for the majority of transfer 
items. This pattern mirrored the results of the correlational analysis in Experiment 2 that a highly positive within-context correlation $(r=.898)$ was obtained between the knowledge partitioning group and the single-context conditions and a moderate negative correlation $(r=-.376)$ was found between contexts.

\section{Contingency Ratings}

Contingency ratings were analyzed by expressing responses as the judged probability of an item belonging to category A. Table 4. $\equiv$ ows the average and two-subgroups ratings for all tested levels of the three dimensions (X, Y, and context) together with their normative values (computed for the overall set of training items). In order to determine whether people's judged contingencies departed from chance, the mean response at each value of each dimension was compared to .5 by a single-sample t-test. Using a significance level of .05 for each test, 6 dimensional values (100, 300 and 700 on dimension $\mathrm{X}$ and 200, 400 and 800 on dimension Y) were found to depart from chance.

\section{Insert Table 4.5 about here}

Specifically, there was some suggestion that people were sensitive to the role of dimensions $\mathrm{X}$ and $\mathrm{Y}$, but their judged contingencies clustered relatively close to chance and were not nearly as extreme as the normative values. For the context dimension, there was no evidence that people erroneously judged it to be predictive, as their responses were close to the normatively correct chance value (largest $\underline{\mathrm{t}} \approx 1.00$ ) and also did not differ between the two contexts $[\underline{\mathrm{t}}(15)$ $<1]$. This rating result was unchanged across the KP and TB groups. Thus, participants used context to gate use of partial knowledge, not to directly predict outcomes.

The ratings for the predictor $\mathrm{X}$ and $\mathrm{Y}$ show a great between-subgroups difference. The participants in the knowledge partitioning group made ratings not too much different from .5 along values on dimensions $\mathrm{X}$ and $\mathrm{Y}$. However, the true-boundary participants made a high variety of ratings across values on both dimensions. Specifically, the degree of the deviation is 
larger for dimension $\mathrm{X}$ than for dimension $\mathrm{Y}$. This result reflects what Erickson and Kruschke (1998, Appendix C) reported, with the same stimuli as used in this experiment, that people attend to the bar-segment dimension more than the height dimension.-Some researchers also regard this result as the differential salience between these two dimensions (Kruschke \& Johansen, 1999). It follows that the partitioning participants did not put as much attention to both dimensions as did the true-boundary participants. This is reasonable, because they relied on context to choose a suitable boundary for categorization. It suggests that the partitioning participants should shift a bit attention from dimensions $\mathrm{X}$ and $\mathrm{Y}$ to context (the modeling results in Chapter 6 confirmed this idea). Also, the probability of response A would increase in Area 1 in the lower context and Area 3 in the upper context due to the application of different boundary. Thus, the ratings for those extreme values on both dimensions $\mathrm{X}$ and $\mathrm{Y}$ which correspond to Area 1 and Area 3 deviate from their normative probabilities (.00) more than did by the true-boundary participants.

\section{Summary of The Results From All Six Experiments}

All six experiments showed that people can learn to use different knowledge in different contexts for categorization, and that once a rule is chosen the other rule is completely ignored. This demonstration of knowledge partitioning entails several important component findings: First, people learned to rely on a normatively neutral context cue to develop different categorization strategies in the systematic-context condition. That is people, were sensitive to the manipulated correlation between the context cue and its corresponding categorization boundary. Second, people’s performance at transfer showed notable individual differences. On average, 1/3 of participants showed context-dependent performance, whereas an equal number of participants in the same condition instead learned to ignore the context at all (i.e., Experiment 5). Third, when people partition their knowledge, there is little or no evidence for integration between knowledge parcels. Instead, people seemingly gate their knowledge on the basis of context, even though they are aware that context by itself does not predict category membership, as revealed 
by the correlational analyses in Experiment 2 and 6. Fourth, knowledge partitioning was observed using category learning irrespective of the type of stimuli (i.e., Experiments 1 through 4 used conceptual category learning; Experiments 5 and 6 used perceptual category learning).

These observed results will be examined further through application of existing models of category learning. First, we can rule out two classes of model, either because of the incompatibility with the present category structures or because of the contradiction between the theory predictions and the observed results. This will be addressed in the following section. The candidate models will be introduced in Chapter 5 . The capacities of these models will be examined by modeling the experimental data, which will be displayed in Chapter 6 .

\section{Feature-Based Theories}

Feature-based theories (also known as component cue models; e.g., Gluck \& Bower, 1988; Gluck, 1992; Attention to Distinctive Input [ADIT]; Kruschke, 1996; Attentional Connectionist Model [ACM]; Shanks, 1992) explain categorization by relying on direct connections between each feature and the possible responses. Herein the direct connection can be realized as the strength of a feature to predict a category response. Suppose stimuli composed of two binary dimensions (or features) allowing four different patterns as $(0,0),(0,1),(1,0)$ and $(1,1)$. Suppose further that only $(0,0)$ belongs in category 1 and the others category 2 . A component cue model will learn that the more value 1 is, the more likely category 2 is. In this case, category 1 and 2 can have a clear distinction. This structure is an example of linear-separable structures. However, if category 1 is defined as the same value on two dimensions and category 2 is defined as the remaining pattern (i.e., XOR structure), then the model cannot distinguish these two categories in terms of the above mechanism. Because the most and least number of value 1 both predict the same category. The XOR structure is a non-linear separable structure. Therefore, the component cue model will have difficulty in learning non-linear separable structure.

It turns out that linear decision planes are incompatible with knowledge partitioning. Consider the stimulus space from the first two studies (e.g., Figure 2.1). The observed opposing 
classifications to transfer items in Areas 2 and 4 in different contexts imply that people's representation of the stimulus space was three-dimensional, with the third dimension representing context. Crucially, there is no single linear decision plane within that threedimensional space that can predict the outcome: Instead, the opposing classifications in Areas 2 and 4 mandate a non-linear decision plane that is a projection onto the positive $\mathrm{X}-\mathrm{Y}$ diagonal in one context and a projection onto the negative diagonal in the other. This requires that the plane be twisted by $90^{\circ}$ along its trajectory through the context dimension (see the left panel in Figure 4. $\equiv$ Similarly, the category structure in Experiments 3 through 6 can be formed by rotating the linear decision plane by $180^{\circ}$ from one context to another (see the right panel in Figure 4.2). Thus, Area 1 in one context becomes Area 3 in another and vice versa. Therefore, both category structure in this research are non-linear separable, such that the component cue model should not be able to account for the experimental data.

Insert Figure 4.2 about here

\section{Incompatibility of Configural Processing with Knowledge Partitioning}

In Experiment 4, 5 and 6, training items presented at transfer were classified according to the true boundary only when contexts at training and test were congruent. When test context was incongruent, old conjunctions of $\mathrm{X}$ and $\mathrm{Y}$ were classified exactly like novel transfer stimuli. One interpretation of this sensitivity to partial change is that people considered the stimuli in a holistic or “configural” manner (e.g., Shanks, Charles, Darby, \& Azmi, 1998; Shanks, Darby, \& Charles, 1998). Configural processing refers to the coding of “...stimuli as indivisible elements rather than as combinations of features or elements” (Shanks, et al., 1998, p. 三). Configural processing stands in contrast to elemental processing, which refers to the decomposition of a complex stimulus into its constituent elements or features (Williams, et al., 1994).

\footnotetext{
${ }^{2}$ The asymmetry with which people partitioned their knowledge in Experiments 1 and 2 may mandate
} 
Evidence for configural processing comes from a variety of sources. For example, Williams et al. (1994) showed that the classic blocking effect (e.g., Kamin, 1969) can be eliminated by configural processing. Similarly, Shanks, Darby, and Charles (1998) showed that the effect of interference also depends on the extent to which processing is configural. In their study, the initially learned associations between two predictors and their respective outcome (e.g., $\mathrm{P} \rightarrow \mathrm{A}$ and $\mathrm{Q} \rightarrow \mathrm{B}$ ) were found to be intact after interpolated learning during which an opposing outcome (A-) was associated with a compound stimulus involving the initial predictors (e.g., $\{\mathrm{P}$, Q $\rightarrow$ A-). This contrasts to the known fact that interference would result if a single predictor were re-assigned during interpolated learning (e.g., $\mathrm{P} \rightarrow \mathrm{A}$ replaced by $\mathrm{P} \rightarrow \mathrm{A}-$ ). Shanks et al. interpreted their results as evidence of configural processing, such that the compound stimulus $\{\mathrm{P}, \mathrm{Q}\}$ is processed independently of its constituent parts $(\mathrm{P}$ and $\mathrm{Q}$

At a surface level, those precedents relate to the present experiments, which found that disruption of the configurality of a training instance by presenting it in a new context rendered it indistinguishable from completely novel transfer items. However, further examination of the implications of configural processing reveals that this approach, too, is incompatible with the present results.

If people engaged in configural processing, they could have considered the stimuli as one of two possible compounds. First, each item may have formed an integrated triplet consisting of a unique conjunction of $\mathrm{X}, \mathrm{Y}$ and Context. Because configural processing considers every compound as an indivisible unit, a training instance would then become a "new" item when tested in an incongruent context. This was seemingly observed in Experiments 4 and 6. However, other aspects of the data from that experiment rule out this representation. Specifically, consider two training triplets presented in context $\mathrm{C}_{1}$, one located in Area 2 (call that $\left.\left\{\mathrm{X}_{1}, \mathrm{Y}_{1}, \mathrm{C}_{1}\right\}\right)$ and another one in either Area 1 or 3 (call that $\left\{\mathrm{X}_{2}, \mathrm{Y}_{2}, \mathrm{C}_{1}\right\}$ ). If those items were processed as indivisible configural stimuli, what would be the effect of testing them in the incongruent context (i.e., as $\left\{\mathrm{X}_{1}, \mathrm{Y}_{1}, \mathrm{C}_{2}\right\}$ and $\left\{\mathrm{X}_{2}, \mathrm{Y}_{2}, \mathrm{C}_{2}\right\}$ )? Configural theories should expect 
the extent of disruption (or, equivalently, remaining generalization; Gluck, 1991; Shanks \& Gluck, 1994) to be equal for both items. However, in Experiment 4 a change in context did not affect responses to training items in Area 2 (i.e., $\left\{\mathrm{X}_{1}, \mathrm{Y}_{1}, \mathrm{C}_{1}\right\}=\left\{\mathrm{X}_{1}, \mathrm{Y}_{1}, \mathrm{C}_{2}\right\}$ ) whereas it had a large effect in Areas 1 and 3 (hence, $\left\{\mathrm{X}_{2}, \mathrm{Y}_{2}, \mathrm{C}_{1}\right\} \neq\left\{\mathrm{X}_{2}, \mathrm{Y}_{2}, \mathrm{C}_{2}\right\}$ ). This appears incompatible with a configural representation involving unified triplets.

A second possibility is that, instead of forming triples, people treat $\mathrm{X}$ and $\mathrm{Y}$ as a configural stimulus (call that $\mathrm{P}$ ) that is accompanied by a (componential) context. A training item in context $\mathrm{C}_{1}$ can thus be represented as $\mathrm{P}+\mathrm{C}_{1}$. Supposing that this item belongs to category $\mathrm{A}$, the learned association can be represented as $\mathrm{P}+\mathrm{C}_{1} \rightarrow \mathrm{A}$. Suppose furthermore that there is another compound of $\mathrm{X}$ and $\mathrm{Y}$ (call that Q) that is associated to the other category in the other context (i.e., $\mathrm{Q}+\mathrm{C}_{2} \rightarrow \mathrm{B}$ ). It follows that when old items are presented in an incongruent test context (i.e., $\mathrm{P}+\mathrm{C}_{2}$ or $\mathrm{Q}+\mathrm{C}_{1}$ ), they should be classified into either category with equal probability. This is because the association of $\mathrm{P}$ to category $\mathrm{A}$ is equivalent to the association of $\mathrm{C}_{2}$ to $\mathrm{B}$, thus predicting either category with equal probability for $\mathrm{P}+\mathrm{C}_{2}$ (and $\mathrm{Q}+\mathrm{C}_{1}$ ). The observed data disconfirm this prediction because people reversed their classification judgment for training items when the context became incongruent at test. It follows that people did not form a partially configural representation. ${ }^{3}$

Neither feature-based theories nor configural processing theories can be a suitable account for the data in this research. In the next chapter, other models (e.g., instance models, general recognition theory and hybrid models) that are not restricted to linear category structure will be discussed for their compatibility with knowledge partitioning. Therefore, the discussion direction shifts to other classes of theories which are either historically relevant to categorization or potentially capable of accounting for knowledge partitioning in categorization. In the next chapter, two types of categorization model, the exemplar-based model and the hybrid model,

\footnotetext{
${ }^{3}$ An objection to this argument might assume that $\mathrm{C}$ is somehow more "salient" than $\mathrm{Q}$ or $\mathrm{P}$, in which case categorization may indeed reverse with context. This objection runs counter to the fact that $C$ on its own, unlike $\mathrm{P}$ or $\mathrm{Q}$, is never a valid predictor during training, which renders it unlikely to be more salient than the accompanying valid compound predictor. See in Table 3.5 and Table 4.6.
} 
will be discussed. Specifically, focus will be put on ALCOVE (an exemplar-based model) and ATRIUM (a hybrid model), given that both models are the candidates to account for knowledge partitioning and their modeling results will be reported in Chapter 6 . 


\section{Chapter 5: Computational Models of Categorization}

The first four chapters have provided very clear evidence of knowledge partitioning in categorization. The next concern is whether or not any of the current computational models can give a good account for these data. As the discussion in Chapter 4 suggests, the component cue model and configural-processing model are not suitable candidates to explain knowledge partitioning. The former model is rejected because of its incompatibility with the non-linear structure of the present experiments. The latter model is rejected because participants did not show a tendency to treat stimuli as any specific configuration at transfer. The rule-based model (e.g., GRT) can also be ruled out to be a candidate model for knowledge partitioning. This is because that the rule-based model did not hold any possibility for the context-dependent use of partial boundaries.

However, there are other categorization models that may be able to accommodate the present data, such as the exemplar-based model and the hybrid model. There is not too much disagreement with that the GCM and ALVOE can be the instances for the exemplar-based model. For the hybrid model, although there are a number of different hybrid models, ATRIUM is picked up for discussion, given that the architecture of ATRIUM - mixture-of-experts - is thought to be the most probable metaphor for knowledge partitioning framework. In this chapter, the possibility for such models to account for knowledge partitioning in categorization is explored.

\section{Exemplar-Based Models}

The act of categorization can be subdivided into two components (e.g., Massaro \& Friedman, 1990). The first involves accessing the category information, and the second involves using this information to select a response. The first component is referred to as the formation of a category representation. In a rule-based model, such as the general recognition theory (GRT, Ashby \& Gott, 1988), it is assumed that people use a decision bound or rule as the representation 
for category structure. In exemplar-based models, by contrast, the exemplars in categories are assumed to be used as the category representation. The process of responding in exemplar models is also quite different from the rule models. Typically, generation of a category response is based on the relative summed similarity of an item to the exemplars in each category.

In this section, two well-known exemplar-based models will be introduced. The first is the generalized context model, GCM, proposed by Nosofsky (1986), and the second is ALCOVE, Attention Learning Covering Map, proposed by Kruschke (1992). Both have proved very successful in accounting for human categorization data.

\section{The Generalized Context Model (GCM)}

The GCM, is the generalized version of the context model (Medin \& Schaffer, 1978) and was proposed by Nosofsky (1986). The main assumption of the context model of Medin and Schaffer is that people's classification of a stimulus is determined by its similarity to stored category exemplars. The initial empirical work for verifying the context model was limited to using the stimuli along binary-valued dimensions (Nosofsky, 1986). Nosofsky (1984) further linked the context model with research in multidimensional scaling, thus extending the model to continuous-dimensional stimuli. The basic idea of the GCM is that every physical stimulus can be represented by a point in a multidimensional psychological space. This idea was called the deterministic multidimensional scaling approach (cf. Nosofsky, 1992). The GCM assumes that stimulus similarity exponentially decreases as the psychological distance between two stimuli increases, as shown in Equation 5.1.

$$
\eta_{i j}=e^{-c d_{i j}}
$$

where

$$
d_{i j}=\left[\sum_{k=1}^{N} w_{k}\left|x_{i k}-x_{j k}\right|^{r}\right]^{1 / r} .
$$

In Equation 5.1, $\eta_{i j}$ is the similarity between stimulus $i$ and stimulus $j$, and $d_{i j}$ is the distance in multidimensional psychological space between these two stimuli. In Equation 5.2, 
the parameter $c$ reflects the overall sensitivity or the specificity of the stored exemplars in the psychological space. The larger $c$ is, the more the psychological distance between stimuli will be amplified. $N$ is the number of dimensions and $w_{k}$ is the attention weight on dimension $k$. The sum of all attention weights is assumed to equal unity. The parameter $r$ determines the nature of the metric by which distances are expressed. In particular, past research suggested that the cityblock metric (i.e., $r=1$ ) is suitable for the psychologically separable dimensions (e.g., length and orientation), such that the effect of one dimension can be considered independently from the effects of other dimensions (e.g., Nosofsky, 1984). A Euclidean distance (i.e., $r=2$ ), however, is suggested for integral dimensions, such as the brightness and saturation of color (e.g., Nosofsky, 1987). Given the assumption that every stimulus is a point in a psychological space, the coordinate values (i.e., $x_{i k}$ in Equation 5.2) are not physical measurement, but the coordinates transferred from the multidimensional scaling (MDS) solution of the stimuli.

A category response is based on the relative summed similarity of a test stimulus to all exemplars in all categories. Thus, the probability that stimulus $i$ will be classified into category $J$ is given by

$$
P\left(R_{J} \mid S_{i}\right)=\frac{b_{J} \sum_{j \in C_{J}} \eta_{i j}}{\sum_{K}\left(b_{K} \sum_{k \in C_{K}} \eta_{i k}\right)} .
$$

In Equation 5.7, the parameter $b_{J}$ is the response bias to category $J$ and the denominator is the summed similarity of stimulus $i$ to all exemplars. Equation 5.3 shows that response selection in the GCM is probabilistic.

Selective attention. In the GCM, one important assumption is that the psychological space can be adjusted by selective attention. That is, the component dimensions can be selectively attended to when making category decisions. The aim of using selective attention is to optimize categorization performance, such that attention on the more diagnostic dimensions will increase and attention on the less diagnostic dimension will decrease (Nosofsky, 1986, 1987). 
Mathematically, the attention weight $w_{k}$ in Equation 5.2 will get larger if the dimension $k$ is more relevant to categorization and otherwise smaller if the dimension $k$ is less relevant to categorization.

For an example of how selective attention works, suppose a set of stimuli consists of three component dimensions (size, shape, and color, each of which has two discrete values. large vs. small for size, circle vs. triangle for shape, and black vs. white for color). The possible psychological representations of the stimulus structure are shown in Figure 5. attention is equally distributed across the three dimensions, and the structure therefore is a 3-D cube. However, if color were more diagnostic for responding than the other two dimensions, then attention to color dimension will increase, thus stretching that dimension in the psychological representation, which decreases between-category similarity. Conversely, attention to the other two dimensions (shape and size) will be reduced, thus shrinking the representation along those dimensions to increase the within-category similarity, as in Panel B.

Insert Figure 5.0 about here

The principal strength of the GCM is that it can provide good accounts of categorization phenomena that are incompatible with the rule-based account. For example, whereas rules are insensitive to the frequency with which stimuli occur, an exemplar-based model would expect frequently-appearing exemplars to be more typical than other items. Two experiments in the study of Nosofsky (1991) consistently revealed that people's typicality rating (i.e., how representative an item was of its category) was influenced by the frequency of the stimulus presentation, not its distance from the boundary as would be expected from a rule-based model. The GCM predicted these results well. In addition, in the same study, the reaction time for classification was found to vary systematically with the manipulation of the frequency of stimuli presentation. This again seemed to imply a relationship between typicality and frequency. 
It seemed, therefore, that typicality was influenced by stimulus frequency in addition to the similarity relations among stimuli (e.g., Barsalou, 1981). Nosofsky (1988a) verified this idea in two experiments that showed classification accuracy and typicality ratings to increase for exemplars that were presented with high frequency. In addition, both ratings increased for those members of the target category that were very similar to the high-frequency exemplars. A frequency-sensitive version GCM was found to give a good fit of these results.

A more direct comparison between the exemplar account and the rule account was provided by Nosofsky, Clark, and Shin (1989). Nosofsky et al. compared the recognition probability for stimuli in different experimental conditions. Participants in one experiment were explicitly informed of the rule for categorization, whereas participants in another experiment were allowed to generate their categorization strategy freely. The results showed that, even in the ruleinstructed experiment, there was still some evidence for the influence of exemplars. Consequently, a model utilizing a mixture of the rule and the GCM provided an excellent account of these data (Experiment 2).

In addition, the relationship between recognition probability and classification rating in the Nosofsky et al. (1989) study provided evidence for the use of exemplars. There was no significant relationship between similarity and recognition probability when participants were instructed to use a rule. However, when participants were allowed to freely learn about the categories, there was a significant positive correlation between these two measures. In combination, these results suggested that in an unconstrained learning situation, stored exemplars would be used for categorization.

McKinley and Nosofsky (1995) also compared the GCM with GRT and showed that the GCM outperformed GRT even on fitting the data collected from the experiments initially used for verifying GRT.

Challenges to the GCM. Although the GCM has evident problematic to account for the inference-intruded categorization, such as the QUARTER and PIZZA problem (Rips, 1989; for detailed discussion, see Cohen, Nosofksy \& Zaki, 2001; Nosofsky \& Johansen, 2000; Smith \& 
Solman, 1994), herein the relevant challenge comes from the demonstration that the category representations might be heterogeneous. Recall the study of Aha and Goldstone (1992), in which two locally heterogeneous clusters of stimuli were presented for learning (see Figure 2.1). Each cluster could be perfectly divided by a linear boundary perpendicular to the $\mathrm{x}$-axis or the $\mathrm{y}$-axis respectively. According to the GCM's assumption that in order to get optimal categorization, diagnostic dimensions will be selectively attended to, more attention should be devoted to the $y$ axis if one wants to perfectly classify the lower-left cluster in Figure 2.1. Conversely, more attention must be devoted to the x-axis if one wants to get a perfect classification for the upperright cluster. Since stretching one dimension necessarily prevents simultaneously stretching of the other, it is impossible to learn a perfect categorization. However, people showed reasonable classification accuracy of items in such a situation without difficulty. In fact, Aha and Goldstone suggested that the GCM cannot handle the locally heterogeneous classification structure involved in their study, unless the GCM incorporated a separate set of dimensional attention weights for each stored exemplar. Thus, the global optimization of attention weights might be a theoretical shortcoming of the GCM.

The application of the GCM to knowledge partitioning. The global optimization hypothesis in the GCM does not suffice to account for Aha and Goldstone's data. This is because in order to optimally classify the items in both clusters, both dimensions should be attended or stretched. However, the GCM cannot stretch all dimensions at the same time because attention to all dimensions always sum to unity. Although my experimental design is somewhat similar to Aha and Goldstone's (1992), the GCM would not be faced with the same dilemma, because attending to the context dimension can result in a 3-D category structure. Take the parallel-boundaries category structure as an example. In Figure 5. planes separated by context values. Thus, in this case, learning to apply each boundary for categorization in the suitable context is possible. However, this is still a problem for the GCM to explain how and why people pay attention to the context dimension. Perhaps, another exemplar- 
based model, ALCOVE, which adds the learning mechanism into the GCM can provide more information.

Insert Figure 5.1 about here

\section{$\underline{\text { ALCOVE }}$}

Although the GCM can provide quantitative predictions for many categorization phenomena, it embodies no learning mechanism to describe how people acquire the presented exemplars in the first place. To compensate for this shortcoming, researchers have created various different versions of the GCM, varying its similarity computation, the overall sensitivity parameter $(c)$, or the response bias sensitive to the learning sequence (Estes, Campbell, Hatsopoulos, \& Hurwitz, 1989; McKinley \& Nosofsky, 1995; Nosofsky, 1987; Nosofsky, Kruschke, \& McKinley, 1992). However, these attempts appeared not to have been fully successful and have been superceded by the idea of combining the exemplar model with an adaptive neural network model. The most successful pairing of these two model types to date is ALCOVE, which was proposed by Kruschke (1992). ALCOVE is a multi-layered neural network model, with gradient descent learning, involving the use of exemplar-based similarity for categorization. To facilitate the presentation of ALCOVE, I first briefly describe neural network models in general.

The standard back-propagation neural network model. The basic idea of neural network models is to imitate the behavior of organisms via the cooperation between interconnected nodes that are functionally similar to real neurons. The most basic neural network consists of two layers of nodes, one layer for encoding stimuli and the other for generating responses. There are association weights connecting the input and output nodes that are adjusted during learning on the basis of feedback. In multi-layered networks additional "hidden" layer of units are sandwiched between the output and the input layer. 
Any layer of nodes receives the activation from the lower layer and transforms the generated activation to the upper one. A variety of transformation function exists; I will describe the one applicable to ALCOVE below.

One of the important characteristics of neural networks is that they are adaptive to learning situations (c.f., Abdi, Valentini \& Edelman, 1999). The key for this characteristic emerges from the fact that the weights connected all layers are adjusted proportionally to the extent of error made by the model. The error is defined as the difference between the target response and the generated response of the model. Take a multi-layered neural network model for example. Once there is error, the extent of error will be used for adjusting the association weights between the output layer and the highest hidden layer, and then adjusting the weights between the highest hidden layer and the second highest hidden layer, and so on. This manner of learning is called back-propagation (e.g., Rumelhart, Hinton, \& Williams, 1986). By this manner, all the association weights between every two layers will be gradually adjusted until there is no error for any stimulus. Therefore, the neural network models can learn the mapping between stimuli and responses in a task without any initial knowledge. ALCOVE implements a variant of this general architecture that is specific to the categorization task.

The architecture of ALCOVE. In ALCOVE, there are three layers of nodes arranged, as shown in Figure 5. of receiving a stimulus value corresponding to a specific stimulus dimension. For example, if the to-be-learned stimuli are composed of three component dimensions, there are three input nodes, each of which corresponds to one of the component dimensions, with different activation levels representing different values. This encoding scheme is called amplitude coding (Kruschke, 1993). 
The middle layer consists of the hidden nodes, each of which corresponds to an exemplar. As in the GCM, each exemplar is assumed to correspond to a point in a psychological space. There are two ways to choose hidden nodes in ALCOVE. The first is to randomly pick points in a psychological space with the constraint that the hidden nodes should cover all the training instances during learning (e.g., Kruschke, 1990, 1992). The second approach is to make the hidden nodes exactly represent the training instances (e.g., Nosofsky \& Kruschke, 1992). On each trial, the activation of each hidden node $a_{j}^{\text {hid }}$ is a function of its similarity to the current stimulus.

$$
a_{j}^{h i d}=\exp \left[-c\left(\sum_{i} \alpha_{i}\left|h_{j i}-a_{i}^{i n}\right|^{r}\right)^{q / r}\right] .
$$

In Equation 5.4, $h_{j i}$ is the position on dimension $i$ of the $j_{t h}$ hidden node and $a_{i}^{i n}$ is the position on dimension $i$ of the current stimulus and $\alpha_{i}$ is the attention weight on dimension $i$. This Equation is exactly the same as Equation 5.1 and Equation 5.2. The activation of the hidden node generated in ALCOVE is reported by the pyramids in Figure 5.2. The closer a stimulus is to a hidden unit in psychological space, the greater is its activation. The shape of the base of the pyramid is influenced by the metric used for similarity computation ${ }^{1}$. This localized responding region (or receptive field) provides ALCOVE with its exemplar-based characteristic, where each hidden node functions like an individual stored exemplar does in the GCM.

Thus, it is convenient to consider Equation 5.4 in the same way as the equations in the GCM. First, the activation of each hidden node (or the inter-stimulus similarity in the GCM) exponentially decays as the psychological distance from the current stimulus to this hidden node increases. In addition, the overall sensitivity parameter $c$ will influence the steepness of the activation profile of hidden nodes. Subsequently, the larger the $c$ parameter is, the steeper the pyramid is, which means an increase in specificity for the relevant hidden node. Therefore, it is

\footnotetext{
${ }^{1}$ The base plane is actually the receptive field for a hidden node. The shape of the receptive field is relevant to which kind of the metric is used for distance computation. The example in Figure 5.2 uses cityblock metric. If the Euclidean metric is used, the shape of the receptive field is circular.
} 
more likely to have one hidden node most activated by the given stimulus, with other nodes having very low activation level. By contrast, a smaller $c$ means that the activation profile is flatter for each hidden node, with the receptive fields becoming larger and overlapping more each other. In this circumstance, there might be several hidden nodes activated at the same time when a given stimulus is entered into ALCOVE. Thus, the specificity of exemplars decreases.

Each node in the output layer corresponds to a response category. Thus, there are two nodes in Figure 5.2, representing the two categories. The output nodes are activated by the sum of the weighted activation of hidden nodes:

$$
a_{k}^{\text {out }}=\sum_{j} w_{k j} a_{j}^{\text {hid }} .
$$

In Equation 5.5, the activation of every hidden node $j$ is multiplied by an association

weight, $w_{k j}$, between it and the $k_{t h}$ output node and then summed together with other weighted activation. To compare model performance with human performance, the output activation must be mapped onto response probabilities. This is done in ALCOVE using the same choice rule as was used in the GCM. Thus,

$$
\operatorname{Pr}(K)=\exp \left(\phi a_{k}^{\text {out }}\right) / \sum_{k} \exp \left(\phi a_{k}^{\text {out }}\right)
$$

where $\phi$ is a real-valued mapping constant (also called a decision constant). In other words, the probability of classifying the given stimulus into category $K$ is determined by the magnitude of category $K$ 's activation (exponentiated) relative to the sum of all category activation (exponentiated).

The feed-forward component of ALCOVE just discussed can be summarized as follows: When a stimulus is presented to ALCOVE, the input nodes are activated according to the dimensional values of that stimulus. Following this, each hidden node is then activated according to the similarity between the stimulus and the hidden nodes via the mediation of the selective attention to dimensions (Equation 5.4). Then the output nodes are activated by the summed weighted activation across all the hidden nodes (Equation 5.5). The final category 
response of ALCOVE is the probability of each category generated by the ratio of the activation of each output node (exponentiated) relative to the sum of all output activation (exponentiated), with all activation weighted by the decision constant (Equation 5.6).

The learning procedure of ALCOVE. The learning algorithm adopted by ALCOVE is the gradient descent algorithm. Thus, learning will continue for as long as there is error, and the weights are adjusted proportionally to the extent of error. In ALCOVE, two types of weight are learned via this algorithm: the association weights between hidden layer and output layer and the attention weight on each dimension.

The error is defined as the difference between the target response and the generated response. During learning, ALCOVE will be given the correct answer (the desired response) each time after it makes a response. This correct answer is used as a teacher (or target) for comparison with the output activation of ALCOVE. A teacher is represented as an activation vector for showing the correct activation each output node should have. Thus, the error generated on each output node is the difference between the teacher activation and the generated activation on each output node. Then the total error of ALCOVE on each trial is the sum-squared difference to the teacher across all output nodes:

$$
E=1 / 2 \sum_{k}\left(t_{k}-a_{k}^{\text {out }}\right)^{2}
$$

The teacher values are defined as

$$
t_{k}=\max \left(+1, a_{k}^{\text {out }}\right) \text { if the stimulus is in Category } K \text { or }
$$

$t_{k}=\min \left(-1, a_{k}^{\text {out }}\right)$ if the stimulus is not in Category $K$.

As stated in Equation 5.7b, if the generated activation is beyond the teacher activation (i.e., larger than +1 when the teacher value is +1 or smaller than -1 when the teacher value is -1 ), then this situation is not regarded as error and no learning will happen at all. The teacher in this mechanism is called "humble teacher" (Kruschke, 1992a, p. 24).

The adjustment of association weights is according to the extent of the error : 


$$
\Delta w_{k j}^{\text {out }}=\lambda_{w}\left(t_{k}-a_{k}^{\text {out }}\right) a_{j}^{\text {hid }} .
$$

In Equation 5.8, $\Delta w_{k j}^{o u t}$ is the amount of adjustment for the weight between the output node $k$ and the hidden node $j$. The equation indicates that if there is no error (i.e., $t_{k}-a_{k}^{\text {out }}=0$ ), then there is no adjustment on that weight. The association weights are changed so as to increase the probability of making a correct prediction to the same stimulus when it is presented again. As error is gradually reduced through learning, the weights change less and less on any given trial. The parameter $\lambda_{w}$ is the learning rate for the associations (a small positive value). A larger learning rate means the model takes a big step at each time of error descent, whereas a smaller learning rate means a small adjustment at each time of descent.

In addition to the association weights being adjusted by the learning algorithm, the attention weights are also adjusted on the basis of error. That is

$$
\Delta \alpha_{i}=-\lambda_{\alpha} \sum_{j}\left[\sum_{k}\left(t_{k}-a_{k}^{\text {out }}\right) w_{k j}\right] a_{j}^{\text {hid }} c\left|h_{j i}-a_{i}^{i n}\right|,
$$

where $\Delta \alpha_{i}$ is the adjustment of attention on dimension $i$, and $\lambda_{\alpha}$ is the learning rate of attention weights. In the same way as for the learning of the association weights, the larger the learning rate for attention weights, the bigger the step during error descent will take ${ }^{2}$. The adjustment of attention weights is also driven by error such that if there is no error (i.e., $t_{k}-a_{k}^{\text {out }}=0$ ), then no change will be made.

Recall that in the GCM, the attention weights are parameters that are freely estimated during modeling. Thus, the GCM cannot describe how participants learn to distribute their attention on the component dimensions. ALCOVE, contrast, provides a plausible attention learning mechanism.

\footnotetext{
${ }^{2}$ Equation 5.15 is the case when the city-block metric is used (see Kruschke, 1992a for the generalized equation).
} 
Learning to attend to relevant dimensions. The major success of ALCOVE results from its ability to learn to distribute attention along dimensions for optimizing categorization. ALCOVE can provide good fits to human data when learning categories in so called filtration and condensation tasks. Consider a category-learning situation in which a single stimulus dimension is relevant. As shown in the top panels in Figure 5. into two categories by using either the horizontal dimension or the vertical dimension. The task with the category structure in the top panels is called the filtration task, as one dimension is sufficient for correct categorization. In contrast, the stimuli in the bottom panels in Figure 5.3 cannot be separated into two groups through use of one dimension alone. That is, both dimensions need to be attended to in order to make an accurate categorization. The task using this kind of structure is called the condensation task. Past research has shown that the filtration task is easier to learn than the condensation task (Gottwald \& Garner, 1972, 1975; Posner, 1964). Kruschke (1991) empirically replicated this finding with stimuli identical to those used in my Experiment 5 and 6 (i.e., a rectangle with an interior vertical line).

Kruschke furthermore showed that ALCOVE can qualitatively predict the differential difficulty of learning these two tasks. Accordingly, attention is adjusted during learning along individual dimensions (see Equation 5.9) that suit the requirement of the filtration task, but attention cannot facilitate learning of the condensation task. Therefore, ALCOVE cannot learn the condensation tasks as well as the filtration tasks.

Insert Figure 5.3 about here

With the attention learning mechanism, ALCOVE also provided a quantitatively accurate account of the differential difficulty of learning the six category structures reported by Shepard, Hovland, and Jenkins (1961). The GCM has no learning mechanism for attention and thus cannot handle these data. The stimuli used in the study by Shepard et al. consisted of three binary-valued dimensions: size, shape and color. Thus there were eight exemplars in total for 
participants to learn. Six types of category structure were manipulated by these researchers. To learn the Type I structure only one dimension was required. Two dimensions were relevant for classification of these exemplars in Type II. Type III, VI, V and VI all required participants to attend to three dimensions. Shepard et al. found empirically that the order difficulty was I $<$ II $<$ (III, IV , V) $<$ VI. Thus, Shepard et al. suggested that the differential difficulty on learning resulted from the adoption of selective dimensions. ALCOVE consistently replicated the difficulty order of these different categories regardless of whether the hidden nodes were the exact eight exemplars or points randomly covering the psychological space (Kruschke, 1990, 1992a; Nosofsky \& Kruschke, 1992).

Limitations of ALCOVE. Three limitations of ALCOVE are addressed here. The first limitation is that ALCOVE cannot capture the pattern of attention shift when the relevant dimensions are changed during learning. Evidence for this difficulty was provided by the dimensional-relevance shift study of Kruschke (1992b). In Kruschke's study, the stimulus consisted of three binary dimensions. In the reversal-shift condition, the two relevant dimensions were unchanged throughout learning, however the mapped category labels were reversed (i.e., category label A swapped for category B) after the shift. In the relevant condition, the shift was accomplished by maintaining the relevance of one of the old dimensions but substituting the other old dimension with the initially irrelevant dimension. Human data revealed that learning the reversal condition was faster than the relevant condition. However, ALCOVE (even after modification) predicted a reversed learning order.

The second limitation is that ALCOVE is very sensitive to the sequence of stimuli. This shortcoming was reported by Lewandowsky (1995) after investigating the ability of ALCOVE to account for the base-rate neglect effect. This effect was first precedented by Gluck and Bower (1988). These researchers trained people to diagnose fictitious patients as having one of two diseases (i.e., category) on the basis of four symptoms, each of which could take on two values (e.g., fever vs. no fever). In a final test, participants had to judge what proportion of patients with a particular pattern of symptoms would suffer a given disease. The crucial manipulation is that 
one category (Common disease) appears as three times more as the other (Rare disease). Thus, the base rate for both categories is 3:1. People's predictions were affected by the relationship among predictors, in addition to the relationship between each predictor and the categories. Specifically, a critical predictor that was normatively irrelevant (because each disease occurred equally often in its presence) was erroneously judged to be predictive of the rare, neglecting the fact that the common disease appears three times more than the rare one.

ALCOVE was initially shown to be able to account for this effect (Kruschke, 1990, 1992a, 1993; Nosofsky \& Kruschke, 1992; Nosofsky, Kruschke, \& McKinley, 1992). However, Lewandowsky (1995) re-examined the base-rate neglect account of ALCOVE and found that the predicted neglect disappeared if the correct categories for the last two trials were reversed, even though this change did not alter the overall probabilities structure of the sequence. This is because the weight update of ALCOVE is quite sensitive to the change of the correct target on successive trials. Thus, Lewandowsky (1995, p.189) claimed: “even though ALCOVE may predict base-rate neglect when the most recent weight update favored the rare disease, this ability is lost rapidly with reinforcement of the common disease."

The third limitation is that ALCOVE lacks the ability to account for certain heterogeneous category representations. Erickson and Kruschke (1998, Experiment 1) asked participants to learn a rule-determined category structure with a few exceptions (i.e., stimuli which should be classified in one way by application of the dominant rule but in fact were presented with a different correct target) and Erickson and Kruschke found that people made only few exceptionconsistent responses to items very close to the exception exemplar. Because the exceptionconsistent response was not as high as predicted by similarity comparison, ALCOVE was unable to handle this result. Erickson and Kruschke further showed that a hybrid mode - ATRIUMthat provided an exemplar module with a rule-based approach can provide fairly good quantitative predictions.

Further evidence for the inadequacy of ALCOVE to capture heterogeneous category representations can be found in a study by Erickson and Kruschke (2001). These authors 
replicated the work of Aha and Goldstone (1992) using a similar category structure, but with an enriched set of diagnostic transfer items. Erickson and Kruschke also examined participants' individual differences to avoid any artifacts that might arise from averaging the data. The participants' learning performance showed that one dimension (the primary dimension) was learned better than the other (the secondary dimension), with the identity of the primary dimension differing between participants. The participants primarily made primary-ruleconsistent responses and a small number of secondary-rule-consistent responses on the diagnostic items, which were located mid-way between the two clusters of training items. ALCOVE failed to predict this pattern, because it always made a moderate number of responses consistent with each rule (ranging from $48 \%$ to $62 \%$ ). This is because the diagnostic items were designed to be equally similar to the training items in the two clusters. Therefore, although ALCOVE, a single exemplar-based system for categorization, has been providing good accounts of many categorization phenomena, it cannot account for the heterogeneity of category representations.

The application of ALCOVE to knowledge partitioning. The error-driven learning mechanism in ALCOVE can be expected to learn to ignore irrelevant dimensions because attending to them is of no help for categorization. In the present experiments, context itself cannot predict the category responses. It can therefore be expected that ALCOVE would always learn to ignore the context dimension. Thus, ALCOVE should perform like those participants who learned the true boundary. By implication, ALCOVE is unlikely to show the partitioning in category representations. In Chapter 6, ALCOVE was chosen as an example of an exemplarbased model to fit the observed data in my experiments, given that another candidate ATRIUM is actually an extension model of ALCOVE.

\section{ATRIUM: A Hybrid Neural Network Model for Categorization}

ARIUM (Erickson \& Kruschke, 1998, 2001, 2002; Kruschke \& Erickson 1994), with its hybrid rule- and exemplar-based representations, derives from the mixture-of-experts approach 
(Jacobs et al., 1991), which holds that different modules (or "experts") learn independently to predict outcomes, with a gating mechanism deciding the extent to which each module contributes to the final outcome. In ATRIUM, category learning is explained by a minimum of two modules; an exemplar module that consists of ALCOVE, plus at least one rule module. The rule module in ATRIUM implements a one-dimensional linear boundary that divides a dimension into two response regions. If the category space involves multiple dimensions, one rule module exists for each dimension. All modules receive input in parallel and all produce a candidate categorization response. The candidate responses from all modules-including the exemplar module - are combined by a gating mechanism to determine the final output of the model. This gating mechanism is one of the features of ATRIUM that differentiates it from other models of categorization, translating the activation of the output units (also called gate nodes; Erickson \& Kruschke, 2001) into a gain, or choice probability, for the corresponding module. The module's candidate response probabilities are weighted by that gain, and the final response of the system is the sum of those weighted probabilities across all modules.

During training, ATRIUM learns a variety of weights by gradient descent; namely (1) the association weights between the input and output nodes in the two rule modules, (2) dimensional attention in the exemplar module, (3) the weights between hidden nodes and output nodes in the exemplar module, and (4) the weights between gate nodes and hidden nodes in the exemplar module. The latter are of particular importance because they represent knowledge of the extent to which each module contributes to classification of the current item, thus giving rise to what is termed representational attention (Erickson \& Kruschke, 2002).

Representational attention, the ability to classify different stimuli in different ways, is the most crucial attribute of ATRIUM. Thus, when applied to a rule-plus-exception structure like the one used by Erickson and Kruschke (1998, Experiment 2), ATRIUM classifies transfer stimuli on the basis of two distinct types of representation. First, the majority of stimuli in a given response region are categorized on the basis of the one-dimensional rule. Second, stimuli that are located in proximity to the exceptions encountered during training, are classified not on the basis 
of the rule but through use of the exemplar module. The information about how stimuli should be classified is associated with each learned exemplar and is retrieved on the basis of the proximity between a transfer stimulus and the closest trained exemplar(s). The following section introduces the version of ATRIUM used for the present modeling, including the architecture, the feed-forward computation in each module, and the learning algorithms.

\section{The Architecture of ATRIUM}

Since the category structure involved two partial boundaries, the architecture of ATRIUM for modeling the present data consists of one exemplar module, two rule modules and a gating mechanism (as shown in Figure 5. $\equiv$ The exemplar module here can be viewed as ALCOVE, comprising three input nodes, each encoding the stimulus level on a single dimension. The hidden nodes are of equal number to the exemplars and two output nodes, each corresponding to a category. The three stimulus dimensions are denoted as $\mathrm{X}, \mathrm{Y}$ and $\mathrm{C}$ (context).

Insert Figure 5.4 about here

There are two rule modules, each of which deals with the learning of a partial rule implied by the category structure. In the original design of ATRIUM, the rule module can only learn a rule that is perpendicular to a stimulus dimension. This is incompatible with the twodimensional rules of the category structures used in the current research. Thus, in order to implement the rule modules, the two-dimensional stimulus, $(\mathrm{X}, \mathrm{Y})$, was transformed to a onedimensional stimulus, $\mathrm{Z}$, by $\mathrm{Z}=\mathrm{Y}-\mathrm{X}$. Accordingly, the partial rules become one-dimensional. For example, the rule of $\mathrm{Y}-\mathrm{X}=100$ is transformed to $\mathrm{Z}=100$. In modeling the data of Experiment 1 and 2, where the true boundary is bilinear, the upper-rule module learns the left partial rule whereas the lower-rule module learns the right one. Similarly, in modeling the data of Experiment 5 and 6, where the true boundary consists of two parallel rule boundaries, the upper-rule module learns the upper rule whereas the lower-rule module learns the lower one. For 
modeling, the stimulus values $\mathrm{X}$ and $\mathrm{Y}$ were transformed to the range between 0 and 1 , and the context values were also transformed to 0 and 1 respectively.

The model's final response is generated by all modules under the mediation of the gating mechanism, which is formed by connecting the hidden nodes in the exemplar module to three gate nodes, each corresponding to a module. In generating the final response, the activation of each gate node is transformed to a percentage weight, which determines the proportion to which each module contributes to the final response. The forward component of each module and the learning algorithms which were all based on the Erickson and Kruschke $(1998,2001)$ study are in turn introduced.

\section{$\underline{\text { Rule Module }}$}

The rule module contains two layers of nodes, input nodes and output nodes. In Figure 5.4, the input and output nodes in the rule modules are represented by the shaded circles and white

circles respectively. The input nodes implement a linear sigmoid function that is aligned with the rule dimension and has its bias adjusted such that the midpoint of the sigmoid function falls on the rule boundary (see Equation 5.10). As shown in Equation 5.10, the activation of the input node, or the value of the sigmoid function, spreads from 0 to 1 . The larger the activation, the more the value approaches 1 . The activation of the input nodes depend on the rule dimension, namely dimension Z. One node responds to larger values and the other to smaller ones, as follows:

$$
\begin{aligned}
& a_{\text {small }}=1-\left\{1+\exp \left[-\gamma_{i}\left(Z_{i}+\beta_{i}\right)\right]\right\}^{-1} \\
& a_{l \text { arge }}=\left\{1+\exp \left[-\gamma_{i}\left(Z_{i}+\beta_{i}\right)\right]\right\}^{-1} .
\end{aligned}
$$

Here, $\gamma_{i}$ represents the gain of the sigmoid function, $Z_{i}$ represents the stimulus value on dimension $\mathrm{Z}$ and $\beta_{i}$ represents the bias. When ATRIUM was fit to the data, the bias value was set according to the defining value of the rule on dimension $\mathrm{Z}$. For example, if the rule is $\mathrm{Z}=$ 
100 , then the bias $\beta_{i}$ is set to -100 . Thus, when the stimulus value $Z_{i}$ is 100 , the activation of the input node will be .5 , which means the stimulus is located on the rule boundary. Therefore, the larger-value input node will be more activated when $\mathrm{Z}_{i}$ is larger than $\beta_{i}$, in contrast, the smaller-value input node will be more activated when $Z_{i}$ is smaller than $\beta_{i}$. The bias $\beta_{i}$ in this thesis was set as a parameter for estimation, following the design in the Erickson and Kruschke (1998) study, yet it can be learned by gradient decent on errors in a distributed neural network structure, such as the design of ATRIUM in Erickson and Kruschke (2001) study.

These two input nodes both have the weighted connection to the two output nodes, which correspond to two categories. The activation $a_{r_{k}}$ of rule-module output node $k$ is given by:

$$
a_{r_{k}}=w_{r_{k}, l \text { arg } e} a_{l \arg e}+w_{r_{k}, \text { small }} a_{\text {small }},
$$

where $w_{r_{k}, l \text { arge }}$ is the connection weight between the large-value node and the output node $k$ and $w_{r_{k} \text {,small }}$ is the connection weight between the small-value node and the output node $k$.

\section{Exemplar Module}

The exemplar module in ATRIUM is an implementation of ALCOVE. Therefore, the exemplar module contains three layers of nodes: output nodes, hidden nodes and input nodes. There three input nodes each encodes the stimulus value on a stimulus dimension (i.e., X, Y, and C). Each input node directly encoded the transformed stimulus value. The activation $a_{e_{j}}$ of a hidden node $j$ exponentially decays as the distance between the current stimulus and this hidden node increases (see the shaded circles in the exemplar module in Figure 5.4).

$$
a_{e_{j}}=\exp \left[-.5 c\left(\sum_{i} \alpha_{i}\left|h_{e j i}-d_{i}\right|\right)\right] \text {. }
$$

The meanings and the notations of the parameters in Equation 5.12 are same as Equation 5.4. The total attention on all dimensions $\sum_{i} \alpha_{i}$ was assumed to be 1 and the initial attention was assumed to be equally distributed across dimensions $\left(\alpha_{i}=.33\right)$. There are two output nodes 
for two categories. The activation $a_{e_{k}}$ of output node $k$ is the sum of the weighted activation of each hidden node. The computation for activation on output nodes in the exemplar module is as

$$
a_{e_{k}}=\sum_{e_{j}} w_{e_{k}, e_{j}} a_{e_{j}}
$$

where $w_{e_{k}, e_{j}}$ is the association weight between output node $k$ and hidden node $j$. This equation is same as Equation 5.5 used for ALCOVE.

\section{Gating Mechanism}

The job of the gating mechanism is to decide which module should contribute most to the response the current stimulus. Therefore, there are three gate nodes, each corresponding to a module (see gating mechanism in Figure 5.4). The activation $a_{a_{m}}$ of gate node $m$ is the sum of the weighted activation received from all hidden nodes $j$, as follows:

$$
a_{g_{m}}=\sum_{e_{j}} w_{g_{m}, e_{j}} a_{e_{j}}
$$

where $w_{g_{m}, e_{j}}$ is the association weight between gate node $m$ and hidden node $j$. The activation of gate node $m$ will be transformed to the probability $P_{m}$ that module $m$ will be used to make a final classification judgement. As expressed in Equation 5.15,

$$
p_{m}=\frac{\exp \left(\phi_{g} a_{g_{m}}\right)}{\sum_{m} \exp \left(\phi_{g} a_{g_{m}}\right)}
$$

where $\phi_{g} \geq 0$ is a scaling constant. If $\phi_{g}$ is high, differences among the gate-node activations are enhanced in the gate-node probabilities. If $\phi_{g}$ is low, differences among the gate-node activations are attenuated in the gate-node probabilities.

Thus, the final probability $p(k)$ to classify a stimulus to category $k$ is transformed from the activation of output node $k$ in each module (which is weighted by the corresponding gate node), such that:

$$
p(k)=\sum_{m} p_{m} \frac{\exp \left(\phi a_{m_{k}}\right)}{\sum_{k} \exp \left(\phi a_{m_{k}}\right)},
$$


herein $m$ indexes the three gate nodes for $P_{m}$ and the three modules for $a_{m_{k}}$ and $\phi \geq 0$ is a scaling constant.

\section{Learning}

ATRIUM learns by gradient descent of error. The error is the difference between teacher activation and the generated activation on each output node. There is no error when the model's output is "more correct" than the target value. That is, there is no error if the generated activation is greater than the greater target value or if it is less than the lesser target value, expressed as follows:

$$
\begin{aligned}
& t_{m_{k}}=\max \left(1, a_{m_{k}}\right) \text { if } k \text { is correct } \\
& t_{m_{k}}=\min \left(0, a_{m_{k}}\right) \text { otherwise, }
\end{aligned}
$$

where $t_{m_{k}}$ and $a_{m_{k}}$ is the target value and the generated activation for output node $k$ in module $m$. The error $A_{m}$ in module $\mathrm{m}$ is expressed as in Equation 5.18:

$$
A_{m}=\exp \left[-.5 c_{m} \sum_{k}\left(t_{m_{k}}-a_{m_{k}}\right)^{2}\right] \text {, }
$$

where $c_{m} \geq 0$ is the cost of module $m$ and represents two factors assumed to be relevant to learning. One is the difficulty or the duration of applying the module and the other is the learners' propensity to use the module for categorization (Erickson \& Kruschke, 2001). In the simulation of the experiments in this research, $c$ was set to 1 for each module, given that the difficulty of applying all modules was assumed to be of no consequence. The mean error of ATRIUM is the sum of the error of each module multiplied by its chosen probability for response, as

$$
\bar{A}=\sum_{m} p_{m} A_{m}
$$


Learning equations for the association weights and attention strengths for Equation 5.12, 5.13, and 5.14 can be derived using gradient descent of error. The adjustment made for the association weight between input node $j$ and output node $k$ in rule module $r_{i}$ is expressed as

$$
\Delta w_{r_{i k}, r_{i j}}=\lambda_{r_{i}} \frac{p_{r_{i}} A_{r_{i}} c_{r_{i}}}{\bar{A}}\left(t_{r_{i k}}-a_{r_{i k}}\right) a_{r_{i j}}
$$

where $\lambda_{r_{i}} \geq 0$ is a freely estimated constant of proportionality called the rule learning rate. The learning equation for the weights between output node $k$ and hidden node $j$ in the exemplar module is expressed as:

$$
\Delta w_{e_{k}, e_{j}}=\lambda_{e} \frac{p_{e} A_{e} c_{e}}{\bar{A}}\left(t_{e_{k}}-a_{e_{k}}\right) a_{e_{j}}
$$

where $\lambda_{e} \geq 0$ is a freely estimated constant of proportionality called the exemplar learning rate. The attention strength on dimension $i$ is adjusted by using the equation

$$
\Delta \alpha_{i}=-\lambda_{\alpha} \sum_{e_{j}}\left[\sum_{e_{k}} \frac{p_{e} A_{e} c_{e}}{\bar{A}}\left(t_{e_{k}}-a_{e_{k}}\right) w_{e_{k}, e_{j}}\right] a_{e_{j}} c\left|h_{e_{j i}}-d_{i}\right|,
$$

where $\lambda_{\alpha} \geq 0$ is a freely estimated constant of proportionality called the attention learning rate.

Finally, the equation for learning the association between the hidden nodes in the exemplar module and the gate nodes in the gating mechanism is expressed as follows:

$$
\Delta w_{g_{m}, e_{j}}=\lambda_{g} \sum_{M} \frac{A_{M}}{\bar{A}}\left(K_{M m} p_{M}-p_{M} p_{m}\right) \phi_{g} a_{e_{j}}
$$

In Equation 5.23, $\lambda_{g} \geq 0$ is a freely estimated constant of proportionality called the gate learning rate, and the value of $K_{x y}$ will be 1 if $x=y$, otherwise 0 . Although the final output of the model is a mixture of the weighted predictions from all modules, this equation entails that each module competes with the others for responding to the stimulus (see Erickson \& Kruschke, 2001; Jacobs et al., 1991). The nature of these competing characteristic may enable ATRIUM to predict knowledge partitioning.

\section{$\underline{\text { ATRIUM and Knowledge Partitioning }}$}


The architecture of ATRIUM seems quite adequate for the theoretical challenges posed by knowledge partitioning. First, the multiple modules independently learn to predict responses for the stimuli. This is convergent with the knowledge partitioning framework which posits that knowledge parcels are independent of one another. Second, in ATRIUM, a gating mechanism decides how to weight each module according to the exemplar most similar to the current stimulus. This corresponds to one element of knowledge partitioning, that the choice of knowledge parcels depends on the context. Suppose that context contributes most to computing the activation of exemplars; then the gating mechanism would rely on the context dimension most. Thus, it is in principle possible for the gating mechanism to make context-dependent decisions which can perform knowledge partitioning.

The only potential problem for ATRIUM's ability to predict knowledge partitioning arises from the way it generates the overall responses. In ATRIUM, any final response is generated by summing the weighted response of each module. Thus the overall represents a blend of all module responses. This is different from the either-or fashion of choosing modules assumed in knowledge partitioning. The next chapter continues the theoretical analysis presented here by applying ALCOVE and ATRIUM to some of the data from the present experiments. 


\section{Chapter 6: Computer Simulation Results}

The basic principle underlying computer simulation is to treat a model as if it were a human, thus exposing it to the same circumstances as the human subjects and observing whether the model can produce the same responses as the humans. In the case where multiple candidate models exist, the value of each model can be determined by its capacity to replicate human-like performance. Thus, both ALCOVE and ATRIUM were fit to the observed data in order to examine their ability to explain knowledge partitioning. For a thorough comparison, these two models were fit to the data generated from different learning tasks (i.e., the conceptual and perceptual category learning task) with different category structures (i.e., the bilinear and parallel-linear category structure).

The comparison of ALCOVE and ATRIUM is reported in the next section. All modeling results consistently suggest that ATRIUM outperforms ALCOVE in accounting for the knowledge partitioning data.

\section{Applying ALCOVE to Knowledge Partitioning}

As outlined in the preceding chapter, if ALCOVE is forced to direct a lot of attention towards context, over-and-above that allocated to the other two dimensions, then it might be able to account for knowledge partitioning. This would be equivalent to a 3-D representation of the category space, as shown in Figure 5.2, with the two partial boundaries in X-Y space being widely separated along a third (context) dimension. Given sufficient attention to context, this would render all generalizations context-specific, much as was observed in the present experiments. However, it is not altogether clear why ALCOVE would pay that much attention to context, given that context did not directly predict category membership in any of the conditions and given that perfect categorization was possible on the basis of the remaining dimensions.

For the modeling of ALCOVE, four parameters were freely estimated: the specificity, $c$, the decision constant, $\phi$, the learning rate for association weights between exemplars and output 
nodes, $\lambda_{w}$, and the learning rate for dimensional attention weights represented by $\lambda_{\alpha}$. The detailed meanings of these parameters have been described in Chapter 5. ALCOVE was initialized with all associative weights between hidden nodes and output nodes set to 0 , reflecting the notion that there is no prior knowledge about the relationship between the stimulus dimensions and categories. The initial attention weights were $1 / 3$ for each dimension, reflecting the assumption that attention should be equally allocated to all dimensions before learning begins. For each input node, the physical value on each component dimension was scaled to the range between 0 and 1 .

\section{Modeling The Data From Experiment 1 and 2}

In this research, Experiment 1 included the randomized-context condition whereas Experiment 2 did not. Because more participants showed knowledge partitioning in the systematic-context condition of Experiment 2 than Experiment 1, ALCOVE was fit to the data from the randomized-context condition in Experiment 1 and the systematic-context condition in Experiment 2. The reasons for doing this are (1) that Experiment 2 did not contain a randomized-context condition, (2) that there were too few participants who showed a complete knowledge-partitioning pattern in the systematic-context condition in Experiment 1, and (3) that although ALCOVE was fit to the data from different experiments, they were comparable given that these two experiments in fact used the identical training stimuli. For modeling the results, only those participants were included whose performance was reliably above chance during the final training block. The chance cutoff was .65 $(\approx 26 / 40)$, as derived from a binomial distribution with $\mathrm{p}=.5$ and $\mathrm{n}=40(\alpha=.05)$. The stimulus dimensions used in Experiment 1 and 2 were assumed to be psychologically separable. Thus, the city-block metric was used for computation of the inter-stimulus similarity.

Participants using different categorization strategies cannot be distinguished by their training performances in the present experiments. Thus, if we fit ALCOVE to the training data only and see what ALCOVE predicts, we can be sure what ALCOVE on default would lean in learning the present stimuli (i.e., ignoring the irrelevant context cue or knowledge partitioning). 
Additionally, according to the discussion in Chapter 5 about the exemplar-based model, ALCOVE should always learn the true category boundary and show no knowledge partitioning for the transfer stimuli. Thus, directly fitting ALCOVE to the transfer data of the KP participants can provide us an insight about the maximum amount of knowledge partitioning ALCOVE can predict. Therefore, the computer simulations in this thesis were conducted by fitting a model to the training and transfer data separately.

Fit of ALCOVE to the Randomized-Context Condition in Experiment 1

According to the chance cutoff, the data from 23 participants were considered for the modeling. ALCOVE was fit separately to the training and transfer data.

Fit to training data. ALCOVE was fit to 400 data points representing the average response across participants (40 training items in 10 blocks) by minimizing the negative log of the maximum likelihood (-logL) of the data given the model. The log of the maximum likelihood of the data given the model was computed by

$$
\log L=\sum_{i} \log \left(\sum_{k} f_{i k}\right) !-\sum_{i} \sum_{k}\left(\log f_{i k} !\right)+\sum_{i} \sum_{k}\left(f_{i k} \log p_{i}(k)\right),
$$

where $f_{i k}$ is the observed frequency of the stimulus $i$ being classified in category $k$, and $p_{i}(k)$ is the model's predicted probability that the stimulus $i$ was classified in category $k$.

As shown in Table 6. well. The best-fitting parameter estimates and the fit statistics are shown in Table 6. predicted transfer responses when the model was fit to the training data are shown in the left panel in Figure 6. $\equiv$

Insert Table 6.0 about here

In Figure 6.0. the probability of an A response is displayed by context and area (see Figure 2.2 for definition of areas). The observed data are represented by bars, with the white and shaded bar indicating the left and right context, respectively. ALCOVE's predictions are represented by 
diamonds for the left context and crosses for the right context. The observed data show a trueboundary response pattern, such that the A response was made most in Area 1 but least in the other three areas and the responses in all areas were context-independent.

Insert Table 6.1 about here

Specifically, ALCOVE predicted no difference between contexts in each area. The attention weights on all dimensions changed from .33 to .49 (dimension X), .51 (dimension Y) and 0 (context) during learning. Thus, ALCOVE, like participants, learned to ignore context for categorization and reflected knowledge of the true boundary.

Insert Figure 6.0 about here

Fit to transfer data. ALCOVE was also fit directly to the 40 data points (20 transfer items in 2 contexts) from the transfer test using the same fit. The parameter estimates and fit statistics are shown in Table 6.1. The predicted transfer data are presented as the right panel in Figure 6.0. Not surprisingly, ALCOVE predicted the responses better on direct fit to the transfer data. The learned attention weights were .48 on dimension $\mathrm{X}, .46$ on dimension Y and .06 on context. Thus, ALCOVE can accommodate the data in the randomized-context condition in Experiment 1. Fit of ALCOVE to the Systematic-Context Condition in Experiment 2

In modeling this condition, ALCOVE was fit to the data of the true-boundary (TB) and the knowledge partitioning (KP) groups separately. According to the chance cutoff, the data of 3 participants in the TB group and 4 participants in the KP group were included for modeling.

Fit to the training data. ALCOVE's predicted proportion correct training performance is in Table 6.0. While ALCOVE captured the learning pattern of the KP group quite well, it failed to predict the pattern of the TB group very well. This is because ALCOVE cannot predict the outstanding performance of the 3 true-boundary participants early in training ( $70 \%$ correct in the 
first training block). The best-fitting parameter estimates and the fit statistics are shown in Table 6.1. The predicted transfer responses are shown in Figure 6. in the top and bottom panels, respectively. The top-left panel shows that ALCOVE was able to predict the transfer pattern of the TB group. The attention weights were learned as .52 on dimension $\mathrm{X}, .48$ on dimension $\mathrm{Y}$ and 0 on context. However, ALCOVE was unable to predict the pattern of responses associated with knowledge partitioning, as shown in the bottom-left panel in Figure 6.1. The learned attention weights were .50 on dimension $\mathrm{X}, .50$ on dimension $\mathrm{Y}$, and 0 on context. This finding matches the expectation that the irrelevant dimension will be given less or no attention by ALCOVE.

Insert Figure 6.1 about here

Fit to the transfer data. ALCOVE was fit to the transfer data of both groups separately. The best-fitting parameter estimates and the fit statistics are shown in Table 6.1. The right panels in Figure 6.1 show the performance of ALCOVE. Unsurprisingly, ALCOVE's prediction to the TB group was improved by directly fitting the model to the transfer data. However, ALCOVE could not predict the data of the KP group. In probabilities, if failed to predict the context-dependent reversal on the probability of A responses in the diagnostic Areas 2 and 4. Thus, ALCOVE cannot predict knowledge partitioning even when fit directly to the transfer data.

However, the data in Experiment 1 and 2 might be influenced by numeric labels used in the stimuli which might alter the similarity relationship between items. Thus, the data from Experiment 5 were also chosen to extend the examination of ALCOVE to whether it can account for the perceptual category learning data. This also generalized ALCOVE to different category structure involving two ascending boundaries. 


\section{Modeling The Data From Experiment 5}

It is commonly thought that the stimuli used in Experiment 5 are composed of psychologically separable dimensions (Erickson \& Kruschke, 1998, 2001, 2002), thus, the cityblock metric was used for computing the inter-stimulus similarity.

\section{Fit of ALCOVE to the Randomized-Context Condition in Experiment 5}

According to the chance cutoff, the data from 18 participants in the randomized-context condition were included for modeling. Since the participants in this condition appeared to learn the true boundary, it was expected that ALCOVE would be able to account for their data. The modeling results for the training and transfer data are reported in turn.

Fit to the training data. With attention set to $1 / 3$ for each dimension at the outset of training, ALCOVE provided a good quantitative account of performance when fit to the training data (see Table 6. $\equiv$ The best-fitting parameter estimates and the fit statistic are shown in Table 6. $\equiv \mathrm{D}$ the associated predicted transfer responses are shown in the left panel of Figure 6. $\equiv$ he labeling in Figure 6.2 is the same as in the previous figures in this chapter, except that the context labels are changed to upper and lower context (instead of left and right). As expected, ALCOVE predicted the true-boundary transfer pattern well.

Insert Table 6.2 about here

Insert Figure 6.2 about here

Fit to the transfer data. It can be clearly seen, from the right panel of Figure 6.2, that ALCOVE gave a fairly good prediction of the transfer responses. The best-fitting parameter values and the fit statistics are shown in Table 6.3. Again, it was not surprising that ALCOVE performed very well when fit to the randomized-context condition. 
Insert Table 6.3 about here

Fit of ALCOVE to the Systematic-Context Condition in Experiment 5

There were 10 participants in the TB group and 9 participants in the KP group. None of the participants in either group were excluded by the chance cutoff.

Fit to the training data. Because the two groups cannot be distinguished based on their learning performance, the parameter values used by ALCOVE were constrained to be the same for both groups during the fit to the training data for these two groups. The best-fitting parameter values and the fit statistics are displayed in Table 6.3. ALCOVE provides good accounts of both sets of the training data (see in Table 6.2). The associated transfer predictions are shown in the two left panels in Figure 6. respectively. It is clear that ALCOVE cannot predict knowledge partitioning when fit to the training data. The learned attention weights on dimensions $\mathrm{X}, \mathrm{Y}$, and context were .51, .48, and .01. Consistently with the modeling results in Experiment 2, ALCOVE again learned to ignore the irrelevant context dimension. Thus, ALCOVE on nature would not predict knowledge partitioning.

Insert Figure 6.3 about here

Fit to the transfer data. ALCOVE was therefore fit directly to the transfer responses of the two groups, using a shared estimate of $c$ and $\phi$, but separate estimates for the learning rates $\left(\lambda_{\mathrm{w}}\right.$ and $\lambda_{\alpha}$; see Table 6.3). The predictions are shown in the two right panels of Figure 6.3. The results confirmed the expectation that ALCOVE, would not be able to show knowledge partitioning even when fit to the transfer data of partitioning participants. Presumably, this failure arose because there was no reason for its learning algorithm to recognize the (indirect) 
relevance of context. Examination of the final dimensional attention weights confirmed that when fit to the transfer data, ALCOVE's learned attention weights were .02 for context, and .60 and .38 for $\mathrm{X}$ and $\mathrm{Y}$, respectively.

\section{Individual Differences and ALCOVE}

ALCOVE’s apparent inability to learn to pay attention to context does not necessarily preclude an exemplar-based account of knowledge partitioning. Specifically, it is conceivable that if the model commenced learning with a strong emphasis on context, it might still be able to predict knowledge partitioning. This, in turn, suggests that the individual differences observed in experiments in this research may have arisen from variability among peoples’ preferences for one dimension over the others at the outset of training.

ALCOVE was therefore examined to see if it could capture those individual differences and might, after all, predict knowledge partitioning if it approached learning with an uneven distribution of attention across the three dimensions. Using the parameter estimates from the fit to the training data of the KP and TB groups (see top panel of Table 6.3), 100 simulation runs were conducted, in which the initial attention to context was randomly sampled from a normal distribution for each replication.

The top panels of Figure 6. $\equiv$ ow ALCOVE’s predictions when initial attention to context was sampled from a distribution with mean .15 and standard deviation .07 (initial attention weights were constrained to sum to unity and were equal for $\mathrm{X}$ and $\mathrm{Y}$ ), and the bottom panels show predictions for a distribution with mean .42 and standard deviation .15 . Within each row, the left and right panels show, respectively, predictions for the top and bottom $30 \%$ of the distribution of initial attention to context (and data for the knowledge partitioning and TB group, respectively). From left to right, the obtained average initial attention to context was, respectively, .23 and .05 (top row), and .76 and .25 (bottom row) across panels. 
Notwithstanding the wide range of initial attention to context (viz. from .05 to .76), ALCOVE consistently failed to predict knowledge partitioning. Instead, in all cases, the model either produced transfer responses that resembled those of the TB group, or it produced chancelevel performance (bottom-left panel). Clearly, therefore, ALCOVE cannot capture the individual differences in my experiments by manipulating initial attention to context.

In a final simulation, I set the attention given to context at the outset of training to .8 , and re-estimated the four free parameters while fitting ALCOVE to the transfer data from both groups. Under these circumstances, ALCOVE captured the transfer responses of the KP group well, with $-2 \log L=84.72$ ( $\left.c=9.90 ; \phi=9.54 ; \lambda_{w}=.009 ; \lambda_{\alpha}=.005\right)$. $\equiv$ wever, the extreme attentional starting value for context then prevented ALCOVE from accommodating the TB group, with $-2 \log L=247.10$, notwithstanding the renewed estimate of the four free parameters $\left(c=9.90 ; \phi=6.95 ; \lambda_{w}=.005 ; \lambda_{\alpha}=.017\right) . \equiv$ reason why an extremely high attentional value for context can offer ALCOVE a chance to show knowledge partitioning is relevant to the parameter-estimating process. The reason why ALCOVE with an extremely high attention weight on context can perform context-dependent categorization is that after being reduced by ALCOVE, the remaining attention weight on context would be still large enough. This is also the reason why ALCOVE cannot predict the TB group under the same modeling condition.

\section{$\underline{\text { ALCOVE And Its Limitations }}$}

I have shown that ALCOVE can account for responses that are indicative of learning of the true category boundary. However, except for some very special circumstances, it cannot accommodate knowledge partitioning and it also cannot readily reproduce the individual differences observed in both experiments.

Therefore, we can conclude that knowledge partitioning is not readily explained by exemplar models of categorization. The present data thus challenge Nosofsky and Johansen’s (2000) assertion that exemplar models can account for the majority of categorization data. We now turn to a theoretical alternative that, because it comprises several modules that make 
independent contributions to a categorization decision, seems to be a better candidate for accommodating knowledge partitioning.

\section{Applying ATRIUM to Knowledge Partitioning}

As discussed in Chapter 5, ATRIUM is a likely candidate to account for knowledge partitioning in categorization. The mixture-of-experts architecture is the major reason for this hypothesis. Specifically, instead of using $\mathrm{X}$ and $\mathrm{Y}$ values as input for the rule modules, a module was created that received input according to a compound dimension, $\mathrm{Z}$, defined as $\mathrm{Z}=\mathrm{Y}-\mathrm{X}$. Although this decision removed some potential explanatory power from the theory, by relying on an unspecified translation process, the decision was necessary to permit application of ATRIUM to the present results (M. A. Erickson, personal communication, November 17, 2001). The current version of ATRIUM was thus composed of two one-dimensional rule modules, each of which—via the translation $\mathrm{Z}=\mathrm{Y}-\mathrm{X}$-learned one of the diagonal partial boundaries, plus an exemplar module consisting of ALCOVE, and a gating mechanism that adjudicated between the competing modules. The modeling reported below required 9 parameters in total (see Chapter 5 for the parameters). Unless otherwise noted, the initial attention weights were set to .33 for all dimensions.

\section{Modeling The Data From Experiments 1 and 2}

The modeling exactly paralleled that already reported for ALCOVE. Therefore, the data of all above-chance participants in the randomized-context condition in Experiment 1 and the systematic-context condition in Experiment 2 were considered for modeling.

\section{Fit of ATRIUM to the Randomized-Context Condition in Experiment 1}

Fit to the training data. ATRIUM's predicted proportion correct is shown in Table 6.0. ATRIUM, like ALCOVE, provided a good quantitative account of the training responses. The best-fitting parameter values and fit statistics are shown in Table 6. $\equiv$ he left panel in Figure 6. $\equiv$ shows the predicted transfer responses after fitting ATRIUM to the training data. ATRIUM, clearly predicted the true-boundary response pattern observed in the condition. 
Insert Table 6.4 about here

Insert Figure 6.5 about here

To compare the performance of ALCOVE and ATRIUM, the AIC (Akaike’s Information Criterion statistic, Akaike, 1974), was computed as a measure of the modeling performance. ${ }^{1}$ The AIC measures for ATRIUM and ALCOVE in this condition are shown in Table 6. condition, ATRIUM performed worse than ALCOVE (2004.97 vs. 1885.11).

Insert Table 6.5 about here

This disadvantage for ATRIUM should be due to the computation of the AIC measure, because the difference between these two models was small in terms of the negative log maximum likelihood (993.45 for ATRIUM vs. 938.56 for ALCOVE)².

Fit to the transfer data. The best-fitting parameter values and fit statistics are also shown in Table 6.4. ATRIUM, not surprisingly, closely captured the observed transfer performance when fit directly to the transfer data. Table 6.5 shows that ATRIUM outperformed ALCOVE in fitting to the transfer data (152.69 vs. 188.36). Together with the fit to the training data, there is a small difference between these two AIC measures $(2157.66=2004.97+152.69$ vs. $2073.47=1885.11$ + 188.36). Thus, both models provided equally good accounts for the data from the randomizedcontext condition in Experiment 1. Since ALCOVE can be viewed as a restricted version of

\footnotetext{
${ }^{1}$ The AIC is a measure of the lack of fit between a model and data that includes a penalty for model flexibility as measured by the number of free parameters. The AIC is given by AIC $=-2 \log \mathrm{L}+2 \mathrm{~N}$, where $\log \mathrm{L}$ is the $\log$ of the maximum likelihood of the data given the model, and $\mathrm{N}$ is the number of free parameters in the model.

${ }^{2}$ Although there is not too much difference between ATRIUM and ALCOVE in terms of their maximum likelihood, ATRIUM actually outperformed ALCOVE in terms of their RMSD values (0.05 vs. 0.18).
} 
ATRIUM, this result also suggests that an exemplar-based model is sufficient to account for participants’ performance in the randomized-context condition.

\section{Fit of ATRIUM to the Systematic-Context Condition in Experiment 2}

Although ATRIUM performed slightly worse than ALCOVE when modeling the data from the randomized-context condition in Experiment 1, this disadvantage was mainly a consequence of using more free parameters. The modeling of the systematic-context condition should be more informative for distinguishing these two models, because ALCOVE failed to account for the knowledge partitioning data.

Fit to the training data. As with the modeling of ALCOVE, ATRIUM was fit to the data from the true-boundary and KP groups separately. No parameters were shared for modeling both sets of data, given that the learning performances of these two groups were too varied. Therefore, 9 parameters were freely estimated for each group. Table 6.0 shows ATRIUM’s predicted proportion correct by training block. Basically ATRIUM captured the training pattern well for both groups. The predicted transfer responses are shown in the left panels of Figure 6.

Insert Figure 6.6 about here

The figure shows that ATRIUM always predicts knowledge partitioning, whether it was fit to the learning data of the TB group or the KP group. Given that ATRIUM predicted trueboundary responses when fit to the randomized-context condition, this shows that ATRIUM is sensitive to whether or not the context cue was systematically manipulated during learning. Comparing to ALCOVE, the AIC measure shows that ALCOVE performed slightly better than ATRIUM (1689.26 vs. 1743.5), as in Table 6.5.

Fit to the transfer data. When fit to the transfer data, ATRIUM had no difficulty predicting different transfer patterns for the true-boundary and KP groups (see the right panels in Figure 6.6). The best-fitting estimates and the fit statistics are displayed in Table 6.4. Obviously, ATRIUM outperformed ALCOVE in this condition was (138.27 vs. 354.08, see in Table 6.5), 
even with the penalty of using more parameters. Together with the fit of the training data, ATRIUM $(138.27+1743.5=1881.77)$ provided better accounts of participants’ performance in the systematic-context condition than ALCOVE $(354.08+1689.26=2043.34)$. The reason for the superiority of ATRIUM is that ATRIUM can predict knowledge partitioning, however, ALCOVE cannot.

\section{Modeling The Data From Experiment 5}

As with the modeling of ALCOVE, ATRIUM was fit to the data produced by Experiment 5 for the purpose of a further comparison with ALCOVE. The modeling results for the randomized-context and systematic-context conditions are reported in turn.

Fit of ATRIUM to Randomized-Context Condition in Experiment 5

The data from 18 participants were used for modeling of this condition. ATRIUM was fit to the training data and then the transfer data.

Fit to the training data. ATRIUM predicted the proportion of correct responses quantitatively well (see Table 6.2). The best-fitting parameter estimates and fit statistics are shown in Table 6.7 $\equiv$ he predicted transfer response are shown in the left panel in Figure 6. Again, it comes as little surprise that ATIRUM predicted the true-boundary transfer pattern.

Comparing to ALCOVE, ATRIUM in this condition performed slightly better (1558.29 vs. 1561.28, see Table 6.5).

Insert Table 6.7 about here

Insert Figure 6.7 about here

Fit to the transfer data. ATRIUM was next fit directly to the transfer data. The best-fitting parameter values and fit-statistics are shown in Table 6.7. As usual, the predictions of ATRIUM 
improved when fit directly to the transfer data (see the right panel in Figure 6.7). The modeling performance in this condition also suggests that ATRIUM outperformed ALCOVE (83.51 vs. 136.64). Together with the fit to the training data, both models provided equally good accounts of the responses in the randomized-context condition (1641.8 for ATRIUM vs. 1697.92 for ALCOVE). It is confirmed again that that the exemplar-based model is sufficient to account for participants' performance in the randomized-context condition.

\section{Fit of ATRIUM to Systematic-Context Condition in Experiment 5}

The data from 10 participants in the true-boundary and 9 participants in the KP group were separately fit by ATRIUM.

Fit to the training data. When ATRIUM was fit to the training data, identical best-fitting parameter estimates could be retained for both groups without a loss of fit (see Table 6.7). Table 6.2 attests to the fact that ATRIUM provided a very good account of training performance in this condition. Table 6.5 shows that ATRIUM $($ AIC $=2265.98)$ performed a bit better than ALCOVE $($ AIC $=2352.05)$. Turning to the associated transfer predictions, ATRIUM predicted knowledge-partitioning for both groups of participants as shown in the left panels of Figure 6. This is in striking contrast to the true-boundary transfer pattern that was predicted by ALCOVE under identical circumstances. At the end of training, ATRIUM's learned attention weights for dimension $\mathrm{X}, \mathrm{Y}$, and context were uniformly .33 for both subgroups.

Insert Figure 6.8 about here

Fit to the transfer data. Next ATRIUM was fit to the transfer responses of the two groups. It was possible to obtain a good quantitative fit with 5 parameters kept invariant for both groups and 4 parameters (decision certainty, $\phi$; module probability scaling constant $\phi_{g}$; gate node learning rate, $\lambda_{g}$; attention learning rate, $\lambda_{\alpha}$ ) varying between groups. As shown in the right panels of Figure 6.8, ATRIUM captured performance in both groups with great quantitative precision. 
Comparing the transfer performance of ATRIUM and ALCOVE by computing the AIC across both groups revealed that ATRIUM considerably outperformed ALCOVE (169.64 vs. 301.78, see Table 6.5) despite having 13 instead of 6 free parameters. Again, ATRIUM provided better accounts of participants' performance in the systematic-context condition than ALCOVE $(2265.98+169.64=2435.62$ for ATRIUM vs. $2352.05+301.78=2653.83$ for ALCOVE $)$. This is because that ALCOVE failed to predict knowledge partitioning, however, ATRIUM did not.

Thus far, ATRIUM has evidently been better than ALCOVE in predicting knowledge partitioning. However, when fit to the training data in the systematic-context condition, ATRIUM seems unable to predict distinct performances for the two groups, instead always predicting knowledge partitioning. Thus, it is worth examining whether ATRIUM can predict the individual differences in the systematic-context condition.

Individual Differences and ATRIUM. When fit to the training data of the systematiccontext condition, ATRIUM always predicted knowledge partitioning, even when modeling the performance of the true-boundary participants. Thus, ATRIUM predicts that all participants would partition their knowledge in the systematic-context condition. This is at odds with the experimental data, which show that about $30 \%$ of participants partition their knowledge whereas another 30\% learned the true boundary. The question, of interest, therefore, is whether ATRIUM can capture those individual differences without being given explicit information about peoples' transfer responses?

As with ALCOVE, this issue was examined by initiating training with randomly chosen attention weights for context. Using the parameter estimates from the fit to the training data of the KP and TB groups in the systematic-context condition (see top panel of Table 6.7), 100 simulation runs were conducted with the initial attention to context being randomly sampled for each replication from a normal distribution with mean .15 and standard deviation .07 (initial attention weights were constrained to sum to unity and were equal for $\mathrm{X}$ and $\mathrm{Y}$ ). Figure 6. shows ATRIUM's transfer responses for the top and bottom 30\% (left and right panel, respectively) of the distribution of initial attention to context. The obtained average initial 
attention to context was .23 and .05, respectively, for the left and right panel. The values of the module gains for the top and bottom 30\% were found to parallel the earlier results for the knowledge partitioning and TB groups (see Table 6.7). That is, for replications within the top $30 \%$, context uniformly determined which rule module was applied, whereas for the bottom $30 \%$, it was the area from which test items were sampled and context was largely irrelevant.

Insert Figure 6.9 about here

Figure 6.9 demonstrates very clearly that, unlike ALCOVE, ATRIUM can reproduce the approximate proportion of participants who partition their knowledge (the $30 \%$ of the replications underlying the predictions in the left panel) or learn the true boundary (right panel) by making some very simple assumptions about the distribution of initial attention. When the model randomly emphasizes context at the outset, it partitions knowledge, whereas when it tends to ignore context at the outset, it learns the true boundary. This suggests that the individual differences in these experiments may likewise have reflected random variation of the initial importance that participants attached to the various dimensions.

Another particularly noteworthy aspect of the results in Figure 6.9 is that the variation in initial attention weight gave rise to qualitatively different generalization patterns. That is, the divergence between predictions in the left and right panels occurred despite exposure to an identical training regime and identical parameter settings.

In summary, when modeling the randomized-context condition, both ATRIUM and ALCOVE can give equally good accounts. Also, both models can predict the data of the TB group in the systematic-context condition. However, ALCOVE cannot predict knowledge partitioning whereas ATRIUM can. Additionally, only ATRIUM can show the individual differences displayed by participants during this research. Since ATRIUM and ALCOVE represent an exemplar-based model and a hybrid model with rules and exemplars respectively, 
the present success of ATRIUM challenges Nosofsky and Johansen’s (2000) assertion that exemplar models can account for the majority of categorization data.

\section{$\underline{\text { ATRIUM and Knowledge Partitioning }}$}

Although the success of ATRIUM is impressive, one might wonder why a model in which the output from different modules is blended can perform a categorization task in seemingly either-or manner as assumed by the framework of knowledge partitioning. A good way to answer this question is by examining the gains for the modules in ATRIUM. Take the modeling performance of the systematic-context condition in Experiment 5 as an example. Table 6 . shows the averaged gain for each module when classifying the transfer items in each area and in the test context. The table entities correspond to probabilities individual modules chosen for response.

\section{Insert Table 6.7 about here}

For the KP group, it is clear that the exemplar module was unimportant, irrespective of which area a transfer stimulus fell into and in which context it was presented. Instead, classification was primarily based on the rule modules, with the upper rule module being most important in the upper context and the lower rule module crucial in the lower context. Although this result is not unexpected, given the nature of ATRIUM's predictions, the extreme magnitudes of those gains are noteworthy because they suggest that each rule module is used at the virtual exclusion of any other information. That is, although ATRIUM's gating mechanism is designed to blend output from all modules, in this case there appears to be little blending. Further analysis of the gains during training revealed that the rule modules very rapidly gained prominence, whereas the exemplar module equally quickly lost its importance (see Figure 6. 
For the TB group, by contrast, the distribution of gains was less extreme, although there was a tendency for the upper rule module to be favored for items in Area 1 (irrespective of context), whereas the lower rule module was favored for items in Area 3 (again irrespective of context). Thus, it appears that ATRIUM learned the true boundary by partitioning the category space-albeit not on the basis of context but on the basis of the location of transfer items within $\mathrm{X}-\mathrm{Y}$ space. This result is particularly interesting because it suggests that knowledge partitioning may be central to complex categorization tasks, even if it cannot be detected by behavioral measures. Kalish et al. (in press) presented similar evidence for the centrality of knowledge partitioning in function learning. $\equiv$

\section{Analysis of Weight Changes in ATRIUM}

Since the behavior of each module in ATRIUM is directly determined by the associative weights between output and hidden nodes (in the exemplar module) or output and input nodes (in the rule modules), it is worth examining how those weights changed during learning. ATRIUM’s learning, as with other neural network models, is error-driven. That is, the model adjusts the association weights only when it makes an error. If the error at each time point is connected, then an error surface is generated and the lowest position on this surface is where the system makes minimal error. Thus, learning in a neural network model can be viewed as a tour descending down the error surface. At each time during descent, the direction of movement through across space can be represented by the vector of "weight error derivatives", with the length of the vector indicating the step size (e.g., Lewandowsky, 1995). The vector of weight error derivatives (WEDs) is identified to the right-hand side of the learning Equation 5.20 and 5.21, disregarding the learning rates.

Therefore, the path taken by the system when minimizing error can be outlined by the difference between the descent directions of every two successive WED vectors. This can be 
done by computing the cosine value $(\cos \theta=\mathbf{A} \bullet \mathbf{B} /[\|\mathbf{A}\| \times\|\mathbf{B}\|])$ between every two successive WED vectors (e.g., Lewandowsky, 1995). The range for the cosine is between $1(\theta=0)$ and -1 ( $\theta=180$ ), with orthogonal vector cosine $0(\theta=90)$, respectively. Using the correlation between successive WED vectors, this section analyzes the weight changes when ATRIUM was fit to the transfer data from the true-boundary and KP groups in Experiment 5.

\section{Weight Changes in The Exemplar Module}

The weight changes discussed here were derived from fitting ATRIUM to the transfer data of the TB and KP group in the systematic-context condition. The WED vectors of the association weights between the output and hidden nodes (the right-hand side of Equation 5.20) were included for analysis. The cosine values between every two successive WED vectors in the exemplar module are shown in Figure 6. $\equiv$ As revealed in the Lewandowsky (1995) study regarding the weight changes in ALCOVE, when two successive trials are associated with the same correct answer, the descent direction tends to remain the same, namely the cosine value was close to 1 . When two successive trials entailed different correct answers, the descent direction tended to be reversed, namely the cosine value was close to -1 . Since the present analysis emphasizes the weight changes as a factor of the context, the cosine values for two successive trials with the same correct answer was plotted according to whether or not the context shifted between the two trials ${ }^{3}$. Presumably, if context has no effect, then the cosine value should always be close to 1 .

Insert Figure 6.11 about here

In Figure 6.11, the left and right panels present WED correlations for the TB and KP groups, respectively. Consider first the right panel for the KP group. When context did not shift between trials, the cosine value always was close to 1 , meaning the system descended in a uniform 
direction on the error surface. However, when context shifted between trials, the cosine value dropped dramatically down to about 0 (turning 90 degrees on descent direction). This indicates that learning direction was influenced by context. In addition, this pattern appeared at the first learning trail and was maintained until the last. This implies that the exemplar module did not stop learning to be context-sensitive.

However, a strikingly different pattern can be found for the TB group. The left panel shows that when context did not shift, the descent direction was kept the same. However, the cosine value for the context-shift trials gradually increased from .2 up to .8, which indicates that, with trials, descent direction was gradually less influenced by the context. This is because that ATRIUM needed to decrease attention on context, which received $1 / 3$ of attention at the onset of learning in order to predict the true-boundary performance.

\section{Weight Changes in The Rule modules}

Since the rule module in ATRIUM in this research only considers the $\mathrm{Z}$ value $(\mathrm{Z}=\mathrm{Y}-\mathrm{X})$, it can be assumed that the pattern of cosine values should not vary as a result of context. In each rule module, there are two input nodes and two output nodes. Thus, the WED vectors of the associative weights between the two output and two input nodes (the right-hand side of Equation 5.21) were included for analysis. Figure 6. ATRIUM was fit to both groups. The labeling is the same as used when analyzing the weight changes in the exemplar module, except the black and white squares represent whether two successive trials come from the same cluster or not. In Experiment 5, the training stimuli were classified into the upper and lower clusters, each of which was presented in one color. Therefore, the notation "cluster no shift" in Figure 6.12 means that the two successive trials both come from either the upper or the lower cluster. The notation "cluster shift" means that the two successive trials come from different clusters.

\footnotetext{
${ }^{3}$ Disregarding whether the context shifted, the cosine value in these analyses was close to 1 when two successive trials with the same answer, and closer to -1 when two successive trials with different answers.
} 
The left and right panels present for the TB group and KP groups, respectively, whereas the upper and lower panels show the data for the upper and lower rule module.

Insert Figure 6.12 about here

Unsurprisingly, the cosine value was always close to 1 when there was no cluster shift between trials. The pattern of cosine values when the cluster shifted, however, was very interesting. Two lines were formed by the cosine values when the cluster shifted between trials. One was very close to the cosine value of 1 , nearly overlapping with the line formed by the black squares (no cluster shift). The other spread out in the range between .6 and .8. Further analysis revealed that the lower cosine value always occurred when the rule module had to predict a category B response in the contrast cluster. Take the upper-rule module as an example. According to the upper rule, the items in Area 1 (see Figure 3.1) would be classified into category B and the items in all other areas would be classified as belonging to category A. However, the items in Area 3 should be classified into category B, not category A as predicted by the upper rule; thus, the upper-rule module received an error and then changed the descending direction a bit. The same reason could be applied for the lower-rule module when predicting classification of the items in Area 1.

The changing of direction in the rule modules reveals the limited capacity for each rule module to give accurate predictions. That is, the upper rule could not handle well half of the items in the lower set (the category A item in Area 2) in the category space, and vice versa for the lower rule. Therefore, after competing with each other during learning, the upper-rule module eventually learned to respond to the items near the upper cluster, whereas the lower-rule module learned to respond the items near to the lower cluster. This modular specialization is one of the properties of the mixture-of-experts architecture (e.g., Jacobs, 1997).

Similar results can be found in the Jacobs (1997) study, which showed that this type of specialization is based on the interaction between the task demand and the type of the neural 
network model involved. In Jacobs’ study, a mixture-of-experts model (one module with hidden nodes and the other without hidden nodes) was trained to learn both a recognition task and a spatial location task. Knowing "what" an item was required the memory provided by the hidden nodes, whereas knowing “where” an item was located did not. The simulation results revealed that the module with hidden nodes specialized to provide responses in the recognition task, whereas the module without hidden nodes acquired the role to respond in the location task.

I now present an explanation for why the exemplar module was not chosen for responding by either subgroup. Because the non-linear category structure in Experiment 5 can be separated into two linear structures by either context (for KP group) or location (for TB group), the linearrule modules can learn this non-linear category structure as well. In addition, fewer weights were needed to adjust in the rule modules than in the exemplar module. The rule modules would learn faster than the exemplar module. This can be supported by the gain profiles in Figure 6.10, that the gain for the exemplar module dropped rapidly from the early training. Therefore, the exemplar module lost the competition with the rule modules. Subsequently, knowledge partitioning was accomplished.

\section{$\underline{\text { ATRIUM and Independence of Knowledge Parcels }}$}

A further characteristic of knowledge partitioning is the apparent independence of knowledge parcels (see the earlier correlational analysis of performance in Experiment 2 and Experiment 6). Accordingly, it is worth examining whether ATRIUM can capture this aspect of knowledge partitioning. In order to test this, ATRIUM, with the best-fitting estimates obtained when modeling the knowledge partitioning data, learned the training stimuli in the single-context conditions without further parameter adjustment, and the predicted transfer responses were then used for the correlational analysis. Specifically, ATRIUM's predictions for individual transfer items in the systematic-context condition and the single-context conditions were used to compute the correlations in the same manner as for the behavioral data. In Experiment 2, the predicted within-context correlation between conditions was highly positive $(\underline{\mathrm{r}}=.853)$, whereas the between-context correlation within the systematic-context condition was slightly negative ( $\underline{\mathrm{r}}$ 
$=-.159$ ). The corresponding empirical correlations were .898 and -.376 . The observed betweencontext correlation within the KP group was more negative than the predicted correlation. This may have resulted from an increased context-dependent response pattern in Area 3 shown in the participants' data in Experiment 2 when compared with the modeling data.

In Experiment 6, the predicted within-context correlation between conditions was highly positive $(\underline{r}=.993)$, whereas the between-context correlation within the systematic-context condition was negative $(\underline{r}=-.898)$. This mirrors the pattern of the data (the empirical correlations were .896 and -.738) and further supports the notion that knowledge parcels, as instantiated by the rule modules in ATRIUM, can be largely independent.

There is an additional analysis that can examine the independence of knowledge parcels in the knowledge partitioning framework. If the knowledge parcels are independent of each other, then knowledge gathered separately in different single-context conditions should be able, with combined, to predict knowledge partitioning in the systematic-context condition. Based on this hypothesis, ATRIUM was fit to the training data from the single-context condition first and then, with the same parameter estimates, ATRIUM was asked to predict the transfer data in the systematic-context condition. The data from Experiment 6 were used for this analysis.

Extract knowledge of the partial rules. First, ATRIUM was fit to the training data from each single-context condition in Experiment 6, with both the exemplar module and the contrast rule module switched off, leaving the gating mechanism always to choose the suitable rule module. Thus, the upper-rule module was fit to the data from the upper-only condition and vice versa for the lower-rule module. This was done by fitting each rule module to the training data from the corresponding single-context condition, but the goodness-of-fit index for adjusting parameters was the sum of the modeling performance of both rule modules (each fit to a different data set). There were 4 parameters estimated, with 2 of them shared by both rule modules (the sensitivity parameter $c$ and the decision constant $\phi$ ) and the remaining parameters estimated independently 
(the learning rate for each rule module). The best fit occurred when $c=9.86, \phi=1.14, \lambda_{r_{\text {upper }}}=$ 0.99 , and $\lambda_{r_{\text {lower }}}=1.16$.

The results show that this version of ATRIUM can give good quantitative accounts of the learning performances of both single-context conditions. In Figure 6. 三he solid diamonds represent the observed proportion correct in each learning block, whereas the open circles represent the predictions of ATRIUM. The left and right panel respectively, show predictions for the upper-only and lower-only condition. The maximum likelihood statistics for modeling was 338.22 and 326.53 for the upper-only and the lower-only condition, respectively.

Insert Figure 6.14 about here

Apply the compound of partial knowledge to the systematic-context condition. Using the same parameter values and the associative weights within each rule module from the above modeling, ATRIUM was tested for its ability to predict the transfer responses of the systematiccontext condition in both contexts. A computer simulation was conducted which allowed variation in the gains for both rule modules in order to predict the transfer responses, with the constraint that the sum of the rule gains should be 1. ATRIUM's predictions were obtained for 121 gain-combinations, formed by fully crossing 11 upper-gains in the upper context (range 0 to 1 with steps .1) with 11 upper-gains in the lower context (same range and step size). ${ }^{4}$ The simulation results are displayed in Table 6.

\section{Insert Table 6.8 about here}

The column label represents the gain of the upper-rule module in the lower context, whereas the row label represents the upper-gain in the upper context. The value in each cell is

\footnotetext{
${ }^{4}$ By definition, gains for the lower module are the complement of the pairs.
} 
the maximum likelihood (-2logL) computed from the difference between the predicted and observed probability of an A response on each transfer item. The best fit of ATRIUM occurs at $(0.9,0.1)$ where the upper-rule module contributed to $90 \%$ of the final response in the upper context, and $10 \%$ of the final response in the lower context. Of course, this is equivalent to that the lower-rule module only contributing $10 \%$ of the system response in the upper context, but $90 \%$ in the lower context. The worst fit occurs at $(0,1)$ where each rule module was chosen for a response in its opposite context. The transfer predictions for the cells with the best fit are plotted in Figure 6.

Insert Figure 6.14 about here

From left to right the panels respectively show the results for $(0.9,0.1)$ and $(1,0)$ in the goodness-of-fit table. When the upper-rule module was weighted 0.9 in the upper context and 0.1 in the lower context, the performance of ATRIUM was most similar to the response pattern observed when knowledge partitioning occurred. The difference between the left panel and the right panel is fairly subtle. In sum, these figures support the assumption that only when the partial rules were chosen exclusively (or nearly so), rather than averaged to predict response, can the knowledge-partitioning response pattern be replicated by the model. In the previous correlational analysis, it was shown that the knowledge gathered from the systematic-context condition could predict the transfer data from the single-context conditions. In the present section, it was evident that knowledge of separably-acquired partial rules could predict the transfer data from the systematic-context condition. Thus, it can be concluded that the KP participants truely did partition their knowledge of categorization in this research.

Knowledge partitioning in the randomized-context condition. When modeling the data from the systematic-context condition, ATRIUM tended to partition the use of rule modules either by location of the items (the TB group) or context (the KP group). This implies that a partitioning 
based on the location of the items might also occur in the randomized-context condition. Therefore, the response pattern in the randomized-context condition might be predicted by applying knowledge of the partial rules adequately for the items in different regions in the category structure.

To explore this possibility, ATRIUM was applied to the randomized-context condition in Experiment 5, with the gains set to be area-dependent. That is, the category space was divided into two areas (upper and lower) by the diagonal line of $\mathrm{Y}=\mathrm{X}+100$. The simulation was conducted in the same manner as for the immediately preceding account of the KP group in Experiment 6, except that the upper context became the upper area and vice versa for the lower context. The goodness-of-fits are shown in Table 6. module contributed $80 \%$ of the final response in the upper area, and $20 \%$ in the lower area (and vice versa for the lower rule). The worst fit occurred when the rule module was chosen in the contrast area. The results of the best fit are plotted in Figure 6.

Insert Table 6.9 about here

Insert Figure 6.15 about here

Therefore, even the response pattern in the randomized-context condition can be predicted by two independent partial rules. This suggests that when participants learned the categories in this research, they may still have partitioned their knowledge according to the areas within which the stimuli located in the category space. Therefore, knowledge partitioning might be a fundamental learning strategy in category learning.

Mixture-of-experts structure. The success of ATRIUM in accommodating the present data is in virtue of mixture-of-experts structure. Similarly, the POLE model adopting mixture-ofexperts structure was evident to account for knowledge partitioning in function learning (Kalish, 
et al., in press). Thus, mixture-of-experts structure should be the key for a model to predict knowledge partitioning. In fact, the concept of mixture-of-experts structure is readily consistent to the assumption of knowledge partitioning. Each module (or experts) in mixture-of-experts structure is actually a knowledge parcel. The operating principle of mixture-of-experts structure that each module independently responds to the stimuli is also consistent to the modularization assumption of knowledge partitioning.

The only difference between ATRIUM and the knowledge-partitioning framework is the way to generate a system response. In ATRIUM, the responses from all modules (or experts) would be "blended" to a system response to the stimulus. On the contrary, knowledge partitioning posits that an appropriate knowledge parcel would be "chosen” for response to the current stimulus. However, a blending model can become a choice model, as long as different modules are extremely favored for their specific stimuli. This is the reason why ATRIUM can perform knowledge partitioning (see the analysis of the module gains in earlier sections). Therefore, an idea model for knowledge partitioning should adopt mixture-of-experts structure. ATRIUM with this structure presumably should be an appropriate candidate model for knowledge partitioning in categorization.

\section{Summary}

It is clear that ATRIUM outperformed ALCOVE on accounting for knowledge partitioning. This result was evident in different types of category learning task with different category structures. Although ALCOVE can improve the prediction by setting a huge attention on context at the outset of learning, it is hard to explain why participants would put so much attention on context before the outset of learning. In contrast, ATRIUM can predict knowledge partitioning in addition to the true-boundary response without the extra assumption about attention to context at the outset of learning.

In addition, ATRIUM, with small variability in initial attention to context can predict the large individual differences in the observed data. Examination of the gains for the modules 
showed that the two rule modules shared the task of providing responses and the exemplar module was not chosen when determining the response. In predicting knowledge partitioning, the choice of the rule modules depended on the context, whereas when predicting the trueboundary response, the choice depended on the location of the item. This in turn suggests that although ATRIUM is a blending model, it actually chose responses from the modules in a manner consistent with the knowledge-partitioning framework. That is, when classifying an item, ATRIUM gave an extremely high gain to the suitable module, but extremely low gains to the others. 


\section{Chapter 7: General Discussion}

\section{Summary and Key Points}

The main purpose of this research was to examine whether knowledge partitioning could be experimentally induced in category learning tasks. To achieve this aim, six experiments were conducted using different category structures (i.e., a bilinear category boundary vs. parallelascending boundary) and different types of category learning tasks (i.e., conceptual vs. perceptual). The main experimental results were as follows.

First, the true category boundary in both category structures could be learned by participants when the third dimensions, context, was randomly paired with training stimuli. Second, the systematic-context manipulation was helpful for inducing knowledge partitioning. On average, about $1 / 3$ of the participants in the systematic-context condition learned the true boundary, while knowledge partitioning was revealed by the other $1 / 3$ of the participants. Third, the correlational analysis of the transfer responses between the systematic-context and singlecontext conditions provided clear evidence for the independence of the knowledge parcels. The behavior of the knowledge partitioning participants in one context was highly correlated with the behavior of the participants who only learned the partial rule in the same context. Thus, the idea of knowledge partitioning, that once a knowledge parcel is chosen for a response, people behave as if they have never learned information contained in other parcels, was confirmed. Fourth, the contingency rating data showed that participants regarded context as not predictive of category responses.

Therefore, all six experiments converged to the conclusion that knowledge partitioning could occur in categorization. That is, participants could use a neutral context cue to choose a suitable rule for categorization as if the other rule had never learned. Two current categorization models, ALCOVE and ATRIUM, were examined for their abilities to accommodate knowledge partitioning. The modeling results were as follows. 
Both ALCOVE and ATRIUM can provide a good account of the true-boundary response. However, ALCOVE cannot predict knowledge partitioning, unless an unreasonable large amount of attention is placed on context at the outset of learning. By contrast, ATRIUM can predict knowledge partitioning well in a normal learning situation (attention equally distributed across dimensions). In addition, when initial attention weights were slightly variable, ATRIUM could predict individual learning differences in the systematic-context condition (from TB to KP) as well as the independence of knowledge parcels (a property of knowledge partitioning). Not only could ATRIUM predict performance in the single-context conditions from the systematic-context condition (i.e., the high within-context correlation between conditions in the modeling results), but also, knowledge of the partial rules learned separately in the singlecontext conditions could replicate the performance in the systematic-context condition (i.e., when the two rule-module gains correctly and extremely favored one of them for use in a suitable context). In all cases, ATRIUM relied primarily on its two rule modules with little or no role for the exemplar module. When modeling the knowledge partitioning data, the two rule modules were chosen on the basis of context; whereas when modeling the true-boundary data, including the data from the randomized-context condition, the choice depended on the location of the item in category space. This further suggests that knowledge partitioning might be common in human learning process.

\section{Limitations of the Present Studies}

An apparent limitation concerns the magnitude of the knowledge partitioning effect. Across experiments, on average, 1/3 of participants partitioned their knowledge when given the opportunity to do so. Another $1 / 3$ of participants applied the true boundary, while the remaining participants were not clearly identifiable as using one or the other strategy. At first glance, it might appear that partitioning, while indisputably present, is of only moderate magnitude. These concerns can be allayed by considering the results of Experiments 2 and 6: The fact that responding based on application of a context-gated partial rule was largely indistinguishable 
from performance after partial training suggests that partitioning in Experiments 2 and 6 was as complete as could be expected. Further, although knowledge partitioning is one of several possible strategies, once chosen, knowledge partitioning was completed.

\section{Relationship with Other Findings}

\section{Complete Partitioning vs. Limited Heterogeneity}

The present results go beyond the limited heterogeneity reported by Aha and Goldstone (1992) because those people who partitioned their knowledge were found to use each partial knowledge component as though the other had not existed. This resulted in contradictions and inconsistencies - that is, opposing classifications of the same item in different contexts - that were absent in the study by Aha and Goldstone (see also Erickson \& Kruschke, 2001). There seem to be no further related precedents in categorization; however, Kalish et al. (in press) reported similar contradictions when people learned functions that contained exceptions. Specifically, one of these experiments used a linearly increasing function that contained three gaps, each of which contained an outlying observation. The three outliers, in turn, defined a (sparse) linearly decreasing function. People were found to extrapolate either on the basis of the increasing or the decreasing function. On any given transfer trial, one or the other learned function was chosen to govern the response, without any apparent attempt at integration between the two conflicting response alternatives. These trial-by-trial contradictions resemble the context-specific opposing classifications in the present experiments.

\section{$\underline{\text { Correlated Features in Categorization }}$}

The context effects in the present studies occurred when the experimental manipulation introduced a correlation with the other predictor(s). There has been much research involving correlated predictors, and it is known that when people learn about a category, they not only learn about each feature independently, but also the relations between them (Medin, Altom, Edelson, \& Freko, 1982; Medin, Wattenmaker, \& Hampson, 1987; Thomas, 1998; Wattenmaker, 1992). This is evident in people's ability to predict the absent feature values of 
Symbols on page 114, paragraph one could not be reproduced in the digital version of this thesis for technical reasons.

Please refer to the physical copy of the thesis, held in the University Library. 
novel stimuli (e.g., Anderson \& Fincham, 1996) or in their ability to predict category membership from correlations among predictors $\equiv$ din et al., 1982). Another illustration of the importance of relationships among features was provided by Goldstone, Medin, and Gentner (1991). In their study, participants were asked to judge whether a target (T) resembled one or the other comparison stimulus (A or B). For example, in one case the target consisted of the three features " $\mathrm{X} \diamond \mathrm{X}$ ”, and the comparison stimuli were “XO $\square$ ” (A) and “ $\square \mathrm{O} \square$ ” (B). People judged the target to be more similar to B, although B-unlike A—shared none of its features with $\mathrm{T}$. Instead, people chose B on the basis of its relational similarity (two identical features bracketing a different feature).

Most relevant here is another consequence of correlations among predictors, namely that people sometimes learn to rely on a normatively irrelevant predictor. For example, Gluck and Bower (1988) trained people to diagnose fictitious patients as having one of two diseases (i.e., category) on the basis of four symptoms, each of which could take on two values (e.g., fever vs. no fever). In a final test, participants had to judge what proportion of patients with a particular pattern of symptoms would suffer a given disease. People’s predictions were affected by the relationship among predictors, in addition to the relationship between each predictor and the categories. Specifically, a critical predictor $\left(\underline{\mathrm{P}}_{c}\right)$ that was normatively irrelevant (because each disease occurred equally often in its presence) was erroneously judged to be predictive of one of the diseases (call that $\underline{\mathrm{R}}$ ). This may be due to a correlation among predictors as when $\underline{\mathrm{P}}_{c}$ occurred with disease $\underline{\mathrm{R}}$ during training, it tended to occur on its own or was accompanied by predictors whose associations to the same category were relatively weak.

The results reported here extend the findings of Gluck and Bower in several ways: First, the conditional probability with which a context occurred given a category was equal across categories [i.e., $\mathrm{P}\left(\right.$ Context $_{\mathrm{x}} \mid$ Category $\left._{1}\right)=\mathrm{P}\left(\right.$ Context $_{\mathrm{x}} \mid$ Category $\left._{2}\right)$ ]. This stands in contrast to Gluck and Bower's (1988) study, where the conditional probability with which $\underline{\mathrm{P}}_{c}$ occurred was greater for category $\underline{\mathrm{R}}$ than the other ( $\underline{\mathrm{P}}_{\mathrm{c}}$ was normatively irrelevant only because the base rates of the two categories differed considerably; hence $\underline{\mathrm{R}}$ stands for "rare” category.) Second, in the study 
by Gluck and Bower the participants were shown to rely on the irrelevant cue at test when it was presented in isolation. In the present experiments, by contrast, people recognized the irrelevance of context on its own (contingency ratings in Experiment 4), but nonetheless used it when it cooccurred with other predictors at test.

\section{Explaining Individual Differences}

Recent research in categorization has often emphasized individual differences (e.g., Lewandowsky et al., 2000; Nosofsky et al., 1989; Nosofsky et al., 1994; Nosofsky \& Palmeri, 1998). Johansen and Palmeri (2002) differentiate between two primary approaches to examining individual differences. One approach relies on a small sample of participants, each of whom provides a large number of observations (perhaps in the thousands), with competing models being fitted to each individual separately. The second approach, followed here, involves testing of a larger number of participants for a single session only, with models being evaluated by how well they account for the variability in responses across participants.

The modeling revealed that ATRIUM, unlike ALCOVE, was able to explain most of the individual differences in the systematic-context condition with simple assumptions about the initial distribution of attention (see also Erickson \& Kruschke, 2001). A particularly noteworthy aspect of this result was the emergence of qualitatively different categorization strategies from small initial differences, which resembles a similar observation by Kalish et al. (in press) when their POLE model was applied to the responses of individual participants in function learning experiments.

One limitation of the modeling of individual differences is that its crucial assumption — that slight differences in attention at the outset can lead to very divergent strategies—cannot be empirically verified within the present experiments. Other precedents in the literature, however, suggest the plausibility of this assumption: For example, Barsalou (1983) investigated the formation of ad hoc categories by presenting participants with four exemplars from different common categories and asking them to provide a category label suitable for description of these 
exemplars. Most interesting here is the result that the instance-to-concept associations for those ad hoc category increased in strength through repeated processing (cf., Barsalou, 1983; see also Barsalou, 1985, 1991). This shows that people can spontaneously create an ad hoc category for temporary use, and that this ad hoc category can gather strength and become well-defined with practice. Applied to the present experiments, this implies that participants who partitioned their knowledge may have formed two ad hoc categories, each describing stimuli of one context. Indirect support for this contention was provided by Barsalou (1991), who showed that construction of ad hoc categories often subserves the achievement of a particular goal. Once a goal has been formulated, it guides the search for exemplars that satisfy the constraints. Likewise, participants in the present experiments, upon noticing the grouping of stimuli by context, may have chosen to explore the possibility of classifying the stimuli in some way involving context, thus giving rise to two ad hoc categories based on context.

\section{Context in Knowledge Partitioning}

In this thesis, a salient characteristic of knowledge partitioning is participants' reliance on context while classifying items. Why did people rely on context to generate their categorization strategy? It is implied that relying on context can help people to simplify the task (see Lewandowsky, et al., 2002). First, the learning curves in this thesis and in the study of Lewandowsky, et al. (2002) both showed that people in the systematic-context condition learned more quickly than in the randomized-context condition. Visual inspection on the learning curves of those KP participants revealed that people first selectively focused on the stimuli in one context to learn until they got a reasonable accuracy, then shifting to learn the stimuli in the other context. Thus, people can spend less cognitive resource on each trail and still get a perfect accuracy in learning.

From the perspective of the cue-learning paradigm, context is in fact a part of a stimulus. Nevertheless, there is no conflict between this perspective and the notion about knowledge partitioning in this thesis. First, a number of researchers have asserted that one feature of a 
stimulus can act as context for others of the same stimulus (Medin \& Schaffer, 1978; Nosofsky, 1984, 1986). Second, Kalish et al. (2003) showed that people could partition their knowledge about the to-be-learned function by particular values of the stimuli. In their case, the stimulus values actually played a role of context. Namely, any stimulus feature or value can be context for partitioning, as long as the second-order relationship between this feature and a particular set of stimuli is identified.

It is this second-order relationship a key for ATRIUM to predict knowledge partitioning. Although context is just a stimulus feature (or dimension), the gating mechanism of ATRIUM honestly reflects the perfect correlation (designed by the experimenter) between context and the particular sets of stimuli. Thus, context would be relied on most for ATRIUM in order to distinguish different stimuli. The distinction between different sets of stimuli is done when ATRIUM knows which module should be used to classify which set of stimuli. Thus, once the distinction is done, knowledge partitioning is completed.

\section{Increasing Knowledge Partitioning}

The individual differences in the present experiments highlighted the fact that knowledge partitioning is one of several strategies to learn categories and some people chose it but some people did not. The modeling further suggested that the individual differences could be a function of the amount of initial attention on context. Therefore, a way to increase knowledge partitioning is to increase attention on context at the onset of learning. One way to achieve this aim would be to introduce inferential relationships to the stimulus cues (or dimensions).

As past research has showed, world knowledge about the theoretical or inferential relationships between features does influence category constructing. For example, Wattenmaker, Dewey, Murphy, and Medin (1986) showed that world knowledge can influence the analysis of category features, which in turn can alter the learning difficulty of categories. These researchers asked participants to classify stimulus people into two categories according to their preferred activities (i.e., category features), such as golf and gymnastics. All features could be analyzed 
based on knowledge of the sporting activities (sports vs. non-sports) and of the common location of the activities (indoor vs. outdoor). Thus, when analyzed by the theme of indoor/outdoor activities, "golf” and "gymnastics" would be represented as different feature values for categorization. However, the same activities would be represented as the same feature value if they were analyzed by the theme of sporting activities. Two category structures were designed in their study, such that one would be easier to learn if the features were analyzed by the indoor/outdoor activities and the other the sporting activities. The results showed that the learning difficulty of each category structure, as expected, was contingent with the experimental instruction, regardless of whether the category structures were linearly separable or not.

Similarly, Medin et al. (1987) showed that the use of a family resemblance principle could be increased in constructing categories if the features were correlated. Specifically, when those correlated features could have more logical links (i.e., dizziness and earache), the use of family resemblance would be more frequent.

Such theoretical links between context and other predictors were not the focus of this thesis. For example in the perceptual category learning task, it is hard to imagine any logically necessary relationship between the color (context) and height of the rectangle and the bar position. As well, in the conceptual category learning task, the link between the season (context) and the living depth and the food density was not strong. Despite this, knowledge partitioning was still observed. Thus, it follows that the magnitude of knowledge partitioning might be increased if participants can be made to believe that there is some sort of inferential (or logical or causal) relationship between context and other predictors; Of course, context in nature is often irrelevant to the category structure. For example, we can design a cover story to ask participants to judge whether a disease will be healed or not (categories) according to the doses of two different drugs a patient takes (predictor $\mathrm{X}$ and $\mathrm{Y}$ ), with the context varying depending on whether the patient has an allergy to similar drugs. Based on common knowledge, the presence or absence of such an allergy will be considered when prescribing medicine. Thus, such a case might provide participants with greater confidence, allowing them to generate a relationship 
between context and other predictors. In contrast, if the context were the color of the T-shirt the patient was wearing (red or green), such an inferential relationship should make it less likely that participants attend to context.

Given the functionality of this idea, initial investigation might manipulate attention experimentally as a function of the inferential relationship between cues. Subsequently, the relationship between the initial attention to cues and the individual differences in eventual categorization could be empirically examined with human participants.

Other methods that could be employed to increase knowledge partitioning include (1) reminding participants of the potential role of context in categorization, and (2) making the true category boundary harder to learn. These suggestions can be examined in the future research.

\section{Theoretical Implications}

Some of theoretical implications of the present research have already been discussed in Chapter 4, such as the incompatibility of the component cue and configural models to account for knowledge partitioning. Here the discussion will focus on the two categorization models, ALCOVE and ATRIUM, which showed a possibility to account for knowledge partitioning.

\section{$\underline{\text { Exemplar-Based Models of Categorization }}$}

The fact that participants used context to master the present categorization task even though they demonstrably knew that it did not directly predict category membership (contingency ratings in Experiment 4 and 6) presents a particular challenge to exemplar models which assume that people learn to pay attention to relevant dimensions only. Accordingly, the modeling showed that ALCOVE, an exemplar theory, cannot account for knowledge partitioning $\equiv$ rther examination of the model clearly identified a shift of attention away from context during learning as being responsible for this failure. At first glance, one may attribute this failure to the specific learning mechanism in ALCOVE, rather than to exemplar representations in general. However, this possibility was compromised by the further fact that when knowledge partitioning was produced in ALCOVE (by arbitrarily focusing attention on context at the outset and 
estimating parameters from the knowledge partitioning transfer data), the theory simultaneously lost its ability to handle true-boundary behavior. Thus, it appears that exemplar representations can account for the behavior of one or the other of the principal groups of participants in this research, but not for both.

\section{Hybrid Models of Categorization}

The modeling showed that ATRIUM, a hybrid rule-plus-exemplar model of categorization, provides an adequate explanation of knowledge partitioning. Not only did ATRIUM capture knowledge partitioning, it also produced true-boundary behavior when fit to the transfer responses of TB participants. Moreover, the model was able to capture the distribution of individual differences observed in our experiments by randomly varying the initial attention given to context. Slight variations in initial attention engendered qualitatively different ways in which the task was mastered, with the model resembling either the true-boundary or knowledge partitioning group at the end of training. The crucial property of ATRIUM that underpinned its success was the concept of representational attention (Erickson \& Kruschke, 2001), or the ability to use different components of knowledge to classify different stimuli.

Indeed, it turns out that there are several ways in which ATRIUM could have produced similar results. One possible way has already been demonstrated in Chapter 6. Without the exemplar module, ATRIUM still could provide good accounts of both the KP and TB response patterns, with gains appropriately associated with individual rule modules. Similarly, Erickson and Kruschke (2002) stated that there is no in-principle reason why ATRIUM, instead of combining rules with exemplars, could not instead use several gated exemplar modules. $\equiv$ en that much of the learning in ATRIUM consisted of a shift of the gains associated with each module, it is conceivable that a similar account might have been obtainable with two independent exemplar modules, each of which would have learned a rule-like division of the category space, rather than the rule-plus-exemplar mix that is standard in ATRIUM. Therefore, it is argued that what is critical for ATRIUM to account for knowledge partitioning is not the combination of rules and exemplars but the general mixture-of-experts approach (Jacobs, et al., 
1991) that is embodied in ATRIUM. The crucial elements of the mixture-of-experts approach are that there is a gating mechanism that can choose among different modules, and that each module contains partial information about the task at hand.

The idea of multiple modules (or “experts”) also underlies a recent connectionist model of function learning, known as POLE (Population Of Linear Experts; Kalish et al., in press). Similar to ATRIUM, POLE has been shown to handle knowledge partitioning in its explanatory domain, for example the context-dependent partitioning of function learning that was reported by Lewandowsky et al. (2002), plus several other context-independent partitioning phenomena reported by Kalish et al. (in press).

One particularly noteworthy aspect of POLE is that it learns complex functions by assigning different parts of the task to different partial (linear) functions. For example, when people must predict the value of a variable $(\mathrm{Y})$ that is a quadratic function of a continuous stimulus variable $(\mathrm{X})$, the model assumes that people associate sub-ranges of $\mathrm{X}$ with different linear functions (i.e., $\mathrm{Y}=\underline{\mathrm{b}} \mathrm{X}+\underline{\mathrm{c}}$ ) that are chosen, on the basis of error-correction during learning, from a whole population of such linear experts with different slopes (i.e., different values of $\underline{b}$ ) and intercepts (varying $\underline{c}$ ). POLE therefore implements the idea that knowledge partitioning, rather than being a niche phenomenon, is fundamental to people’s ability to learn function concepts. This theme was also present in the application of ATRIUM: The module gain analysis (Table 6.7) revealed that ATRIUM partitioned its knowledge even for predicting the true-boundary response-except that the partitioning was performed on the basis of the area from which stimuli were sampled rather than on the basis of context. This suggests that the partitioning of a complex task into several simpler ones that are learned independently of each other, is as fundamental to categorization as it arguably is to function learning. 


\section{Other Models and Knowledge Partitioning}

In addition to ATRIUM there are two models involving heterogeneous representations in categorization. Nonetheless, as shown in this section, these two models may have difficulty accommodating knowledge partitioning.

\section{$\underline{\text { RULEX }}$}

Nosofsky et al. (1994) proposed a rule-plus-exception model (RULEX) which assumed that people mainly use rules for categorization and only remember those exemplars that cannot be classified by the rules as exceptions. In RULEX, the rules are formed according to a hypothesistesting procedure. The model first searches for a perfect single-dimensional rule for classification. If no perfect single-dimensional rule can be found, then the model searches for imperfect single-dimension rules. If this strategy still fails, the model turns to search for conjunctive rules. In searching for conjunctive rules, pairs of dimensions will be sampled. For example, a sampled conjunctive rule can be "respond Category A, when both the values on dimension 1 and 2 are $1 ”$.

At any stage of searching for rules, once a rule is generated, it will be tentatively maintained for a minimum number of trials (called lower test window by Nosofsky et al.) and if the accuracy cannot exceed a pre-determined criterion (say 60\%), the rule will be discarded. When a rule is maintained for even longer (twice of the number of trials in lower test window) the rule will be stored as a permanent rule as long as it exceeds a much stricter pre-set criterion (say 90\%), or it is discarded.

The model will start the exception-storage process only when either (1) A rule has been established permanently, whether it be single-dimensional or conjunctive, or (2) all singledimensions and pairs of dimensions are exhausted without forming a rule. Thus, the items which cannot be classified by the permanent rule will be stored as exceptions.

The core idea of RULEX is that people form an imperfect rule to classify items whilst also separately storing those exceptions that remain unexplained by the rule. This idea seems quite intuitive, however, it is nonetheless likely that RULEX will have difficulty in accounting for 
knowledge partitioning. This is because RULEX generates suitable rules on the basis of the predictiveness of single dimensions, so therefore it should not be able to choose context as a crucial component of one of its rules. If RULEX somehow learns a linear rule for items in one context, say the upper rule in the upper context, then RULEX has to store the instances with another context as the exceptions. In this circumstance, the accuracy can not exceed 50\% because those exceptions (i.e., items in the lower context) themselves cannot predict the category labels given that there is equal number of training instances in category A and B presented in either context. Therefore, it is unlikely that RULEX can predict the knowledge partitioning data produced by the experiments within this thesis.

\section{$\underline{\mathrm{ACT}-\mathrm{R}}$}

Anderson and Betz (2001) proposed a hybrid model of categorization within the ACT-R framework (Anderson \& Lebiere, 1998). In this model, two modules are assumed to contribute to the learning of categories; one adopts a rule-based approach and the other an exemplar-based approach. The rule module contained in ACT-R is actually RULEX and the exemplar module is the exemplar-based random walk model (EBRW, Nosofsky \& Palmeri, 1997), which is the GCM combined with the Logan’s (1988) instance-based model of automaticity. Logan’s instance-based model states that as performance becomes skilled, the stored exemplars will "win” the race with a general algorithm (like a categorization rule) and performance becomes automatized. Thus, EBRW is an exemplar-based model with the ability to predict the speed of classification response as a function of learning. During learning, one of the two models will tend to have the most success and will be chosen most often. Thus, one model will be chosen for responding more frequently until it becomes the "winner” and dominates categorization.

However, ACT-R may have difficulty accounting for knowledge partitioning. First, the classification of its component models might not rely on an irrelevant context cue. If RULEX was chosen, ACT-R cannot predict knowledge partitioning as RULEX cannot do so. EBRW, like other exemplar-based models, has difficulty explaining why an irrelevant context cue should be attended to for categorization. The choice mechanism also makes ACT-R less likely to 
account for knowledge partitioning. As Anderson and Betz (2001) stated “The decision to use a rule-based or exemplar-based approach is determined by the overall success of these approaches, rather than by the success with respect to a particular stimulus (p.642).” The design of the present experiments ensured that context did not predict category membership. Thus, no contextsensitive strategy should be retrieved in the ACT-R model unless the model can be sensitive to individual stimuli like ATRIUM. In fact, Erickson and Kruschke (2001) doubted the ACT-R model's ability to account for their findings of stimulus-dependent classification for this same reason.

\section{Conclusions}

This thesis has made several empirical and theoretical contributions. Empirically, it was revealed that: (1) People can be sensitive to the correlation between a context cue and other predictor(s) in either a conceptual or a perceptual category-learning task, even if the other predictors alone permit perfect classification of training items; (2) A significant proportion of people use context to partition their knowledge; and (3) When people partition their knowledge, each knowledge parcel is used as though the others do not exist.

At a theoretical level, the following contributions were made: (1) The knowledge partitioning framework was extended from expertise and function learning to categorization; (2) The present results challenge a number of current theories of categorization; (3) A hybrid model, ATRIUM, was shown to accommodate knowledge partitioning without making extreme assumptions about people's initial distribution of attention; (4) Slight differences in people’s preferences at the outset of learning can engender qualitatively different ways in which a complex task is mastered, a result that has been observed with other mixture-of-experts models; and (5) Knowledge partitioning might be fundamental to learning process, given that ATRIUM, without the exemplar module, can even predict the performance in the randomized-context condition. 


\section{References}

Abdi, H., Valentini, D., \& Edelman, B. (1999). Neural networks. California: SAGE Publications, Inc.

Aha, D. W., \& Goldstone, R. L. (1992). Concept learning and flexible weighting. In J. K. Kruschke (Ed.), Proceedings of the Fourteenth Annual Conference of the Cognitive Science Society (pp. 534-539). Hillsdale, NJ: Erlbaum.

Akaike, H. (1974). A new look at the statistical model identification. IEEE Transactions in Automatic Control, 19, 716-723.

Anderson, J. R., \& Betz, J. (2001). A hybrid model of categorization. Psychonomic Bulletin and Review, 8, 629-647.

Anderson, J. R., \& Fincham, J. M. (1996). Categorization and sensitivity to correlation. Journal of Experimental Psychology: Learning, Memory, and Cognition, 22, 259-277.

Anderson, J. R., \& Lebiere, C. (1998). The atomic component of thought. Mahwah, NJ:Erlbaum.

Ashby, F. G., \& Gott, R. (1988). Decision rules in the perception and categorization of multidimensional stimuli. Journal of Experimental Psychology: Learning, Memory, and Cognition, 14, 33-53.

Ashby, F. G., \& Maddox, W. T. (1992). Complex decision rules in categorization: contrasting novice and experienced performance. Journal of Experimental Psychology: Learning, Memory, and Cognition, 18, 50-71.

Ashby, F. G., \& Townsend, J. T. (1986). Varieties of perceptual independence. Psychological

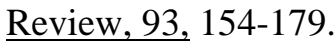

Barsalou, L. W. (1981). Determinants of graded structure in categories. Unpublished doctoral dissertation, Stanford University.

Barsalou, L. W. (1983). Ad hoc categories. Memory \& Cognition, 11, 211-227. 
Barsalou, L. W. (1985). Ideals, central tendency, and frequency of instantiation as determinants of graded structure in categories. Journal of Experimental Psychology: Learning, Memory and Cognition, 11, 629-654.

Barsalou, L. W. (1991). Deriving categories to achieve goals. The psychology of learning and motivation, 27, 1-64.

Bourne, L. E., JR. (1970). Knowing and using concepts. Psychological Review, 77, 546-556.

Busemeyer, J. R., Byun, E., Delosh, E. L., \& McDaniel, M. A. (1997). Learning functional relations based on experience with input-output pairs by humans and artificial neural networks. In K. Lamberts, \& D. Shanks (Eds.), Knowledge, concepts, and categories (pp. 405-435). UK: Psychology Press.

Carraher, T. N., Carraher, D. W., \& Schliemann, A. D. (1985). Mathematics in the streets and in schools. British Journal of Developmental Psychology, 3, 21-29.

Cohen, A. L., Nosofsky, R. M., \& Zaki, S. R. (2001). Category variability, exemplar similarity, and perceptual classification. Memory \& Cognition, 29, 1165-1175.

DeLosh, E. L., Busemeyer, J. R., \& McDaniel, M. A. (1997). Extrapolation: the sine qua non for abstraction in function learning. Journal of Experimental Psychology: Learning, Memory, and Cognition. 24, 968-986.

Erickson, M. A., \& Kruschke, J. K. (1998). Rules and exemplars in category learning. Journal of Experimental Psychology: General, 127, 107-140.

Erickson, M. A., \& Kruschke, J. K. (2001). Multiple representations in inductive category learning: Evidence of stimulus- and time-dependent representation. Manuscript submitted for publication.

Erickson, M. A., \& Kruschke, J. K. (2002). Rule-based extrapolation in perceptual categorization. Psychonomic Bulletin and Review, 9, 160-168.

Ericsson, K. A. (1996). The acquisition of expert performance: An introduction to some of the issues. In K. A. Ericsson(Ed.), The road to excellence: The acquisition of expert performance in the arts and sciences, sports and games(pp. 1-50). Hillsdale, NJ: Erlbaum. 
Estes, W. K. (1993). Models of categorization and category learning. The psychology of learning and motivation, 29, 15-56.

Estes, W. K. (1994). Classification and cognition. New York: Oxford University Press.

Estes, W. K., Campbell, J. A., Hatsopoulos, N., \& Hurwitz, J. B. (1989). Base-rate effects in category learning: a comparison of a parallel network and memory storage-retrieval models. Journal of Experimental Psychology: Learning, Memory and Cognition, 15, 556-571.

Glaser, R. (1996). Changing the agency for learning: Acquiring expert performance. ion to some of the issues. In K. A. Ericsson(Ed.), The road to excellence: The acquisition of expert performance in the arts and sciences, sports and games(pp. 303-311). Hillsdale, NJ: Erlbaum.

Gluck, M. A. (1991). Stimulus generalization and representation in adaptive network models of category learning. Psychological Science, 2, 50-55.

Gluck, M. A. (1992). Stimulus sampling and distributed representation in adaptive network theories of learning. In A. F. Healy, S. \& M. Kosslyn, (Eds.), Essays in honor of William K. Estes: Vol. 2. From learning processes to cognitive processes (pp. 169-199). Hillsdale, NJ: Erlbaum.

Gluck, M. A., \& Bower, G. H. (1988). From conditioning to category learning: an adaptive network model. Journal of Experimental Psychology: General, 117, 227-247.

Goldstone, R. L., Medin, D. L., \& Gentner, D. (1991). Relational similarity and the nonindependence of features in similarity judgement. Cognitive Psychology, 23, 222-262.

Gottwald, R. L., \& Garner, W. R. (1972). Effects of focusing strategy on speeded classification with grouping, filtering and condensation tasks. Perception \& Psychophysics, 11, 179-182.

Gottwald, R. L., \& Garner, W. R. (1975). Filtering and condensation tasks with integral and separable dimensions. Perception \& Psychophysics, 18, 26-28.

Jacobs, R. A. (1997). Nature, nurture, and the development of functional specialization: A computational approach. Psychonomic Bulletin and Review, 4, 299-309. 
Jacobs, R. A., Jordan, M. I., Nowlan, S. J., \& Hinton, G. E. (1991). Adaptive mixtures of local experts. Neural Computation, 3, 79-87.

Johansen, M. K., \& Palmeri, T. J. (2002). Are there representational shifts during category learning? Cognitive Psychology, 45, 482-553.

Kalish, M. L., Lewandowsky, S., \& Kruschke, J. K. (in press). Population of linear experts: Knowledge partitioning and function learning. Psychological Review.

Kamin, L. J. (1969). Predictability, surprise, attention, and conditioning. In B. A. Campbell \& R. M. Church (Eds.), Punishment and aversive behavior (pp. 279-296). New York: AppletonCentury-Crofts.

Kruschke, J. K. (1990). ALCOVEL a connectionist model of category learning. (Indiana University Cognitive Science Report 19). Bloomington: Indiana University.

Kruschke, J. K. (1991). Dimensional attention learning in connectionist models of human categorization. (Indiana University Cognitive Science Report 50). Bloomington: Indiana University.

Kruschke, J. K. (1992a). ALCOVE: An exemplar-based connectionist model of category learning. Psychological Review, 99, 22-44.

Kruschke, J. K. (1992b) Dimensional relevance shifts in category learning. (Indiana University Cognitive Science Report 79). Bloomington: Indiana University.

Kruschke, J. K. (1993). Three principles for models of category learning. The psychology of learning and motivation, 29, 57-90.

Kruschke, J. K. (1996). Base rates in category learning. Journal of Experimental Psychology: Learning, Memory, and Cognition, 22, 3-26.

Kruschke, J. K., \& Erickson, M. A. (1994). Learning of rules that have high-frequency exceptions: New empirical data and a hybrid connectionist model. In A. Ram \& k. Eiselt (Eds.), Proceedings of the Sixteenth Annual Conference of the Cognitive Science Society (pp. 514-519). Hillsdale, NJ: Erlbaum. 
Kruschke, J. K., \& Johansen, M. K. (1999). A model of probabilistic category learning. Journal of Experimental Psychology: Learning, Memory, and Cognition, 25, 1083-1119.

Lamberts, K. (1994). Flexible tuning of similarity in exemplar-based categorization. Journal of Experimental Psychology: Learning, Memory, and Cognition, 20, 1003-1021.

Lewandowsky, S. (1995). Base-rate neglect in ALCOVE: a critical reevaluation. Psychological Review, 102, 185-191.

Lewandowsky, S., Kalish, M., \& Griffiths, T. L. (2000). Competing Strategies in Categorization: Expediency and Resistance to Knowledge Restructuring. Journal of Experimental Psychology: Learning, Memory, and Cognition, 26, 1666-1684.

Lewandowsky, S., Kalish, M., \& Ngang, S. K. (2002). Simplified learning in complex situations: Knowledge partitioning in function learning. Journal of Experimental Psychology: General. Lewandowsky, S., \& Kirsner, K. (2000). Expert knowledge is not always integrated: A case of cognitive partition. Memory \& Cognition, 28, 295-305.

Logan, G. D. (1988). Toward an instance theory of automatization. Psychological Review, 95, $492-527$.

Maddox, W. T., Ashby, F. G. (1993). Comparing decision bound and exemplar models of categorization Perception \& Psychophysics, 53, 49-70.

Massaro, D. W., \& Friedman, D. (1990). Models of integration given multiple sources of information. Psychological Review, 97, 225-252.

McKinley, S. C., \& Nosofsky, R. M. (1995). Investigations of exemplar and decision bound models in large, ill-defined category structures. Journal of Experimental Psychology: Human Perception and Performance, 21, 128-148.

Medin, D. L., Altom, M. W., Edelson, S. M. \& Freko, D. (1982). Correlated symptoms and simulated medical classification. Journal of Experimental Psychology: Learning, Memory, and Cognition, 8, 37-50.

Medin, D. L., \& Schaffer, M. M. (1978). Context theory of classification learning. Psychology Review, 85, 207-238. 
Medin, D. L., \& Shoben, E. J. (1988). Context and structure in conceptual combination. Cognitive Psychology, 20, 158-190.

Medin, D. L., Wattenmaker, W. D., \& Hampson, S. E. (1987). Family resemblance, conceptual cohesiveness, and category construction. Cognitive Psychology, 19, 242-279.

Neisser, U. (1987). Introduction: the ecological and intellectual bases of categorization. In U. Neisser (Ed.), Concepts and conceptual development: ecological and intellectual factors in categorization. Cambridge: Cambridge University.

Newell, A., \& Simon, H. A. (1972). Human problem-solving. Englewood Cliffs, NJ: Prentice Hall.

Nosofsky, R. M. (1984). Choice, similarity, and the context theory of classification. Journal of Experimental Psychology: Learning, Memory, and Cognition, 10, 104-114.

Nosofsky, R. M. (1986). Attention, similarity, and the identification-categorization relationship. Journal of Experimental Psychology: General, 115, 39-57.

Nosofsky, R. M. (1987). Attention and learning process in the identification and categorization of integral stimuli. Journal of Experimental Psychology: Learning, Memory, and Cognition, $\underline{13}, 87-108$

Nosofsky, R. M. (1988a). Similarity, frequency, and category representations. $\underline{\text { Journal of }}$ Experimental Psychology: Learning, Memory, and Cognition, 14, 54-65.

Nosofsky, R. M. (1988b). Exemplar-based accounts of relations between classification, recognition, and typicality. Journal of Experimental Psychology: Learning, Memory, and Cognition, 14, 700-708.

Nosofsky, R. M. (1991). Typicality in logically defined categories: Exemplar-similarity versus rule instantiation. Memory \& Cognition, 19, 131-150.

Nosofsky, R. M. (1992). Similarity scaling and cognitive process models. Annual Review of Psychology, 43, 25-53. 
Nosofsky, R. M., Clark, S. E., \& Shin, J. H. (1989). Rules and exemplars in categorization, identification, and recognition. Journal of Experimental Psychology: Learning, Memory, and Cognition, 15, 282-304.

Nosofsky, R. M., \& Johansen, M. K. (2000). Exemplar-based accounts of “multiple-system” phenomena in perceptual categorization. Psychonomic Bulletin and Review, 7, 375-402.

Nosofsky, R. M., \& Kruschke, J. K. (1992). Investigations of an exemplar-based connectionist model of category learning. The psychology of learning and motivation, 28, 207-250.

Nosofsky, R. M., Kruschke, J. K., \& McKinley, S. C. (1992). Combing exemplar-based category representations and connectionist learning rules. Journal of Experimental Psychology: Learning, Memory, and Cognition, 18, 211-233.

Nosofsky, R. M., \& Palmeri, T. J. (1997). An exemplar-based random walk model of speeded classification. Psychological Review, 104, 266-300.

Nosofsky, R. M., \& Palmeri, T. J. (1998). A rule-plus-exception model for classifying objects in continuous-dimension spaces. Psychonomic Bulletin and Review, 5, 345-369.

Nosofsky, R. M., Palmeri, T. J., \& McKinley, S. C. (1994). Rule-plus-exception model of classification learning. Psychological Review, 101, 53-79.

Nunes, T., Schliemann, A. D., \& Carraher, D. W. (1993). Street mathematics and school mathematics. Cambridge: Cambridge University press.

Posner, M. I. (1964). Information reduction in the analysis of sequential tasks. Psychological Review, 71, 491-504.

Rips, L. J., Shoben, E. J., \& Smith, E. E. (1973). Semantic distance and the verification of semantic relations. Journal of Verbal Learning and Verbal Behavior, 12, 1-20.

Rips, L. J. (1989). Similarity, typicality, and categorization. In S. Vosniadou \& A. Ortony (Eds.), Similarity and analogical reasoning (pp. 21-59). Cambridge: Cambridge University Press.

Roth, E. M., \& Shoben, E. J. (1983). The effect of context on the structure of categories. Cognitive Psychology, 15, 346-378. 
Rosch, E., \& Mervis, C. B. (1975). Family resemblances: studies in the internal structure of categories. Cognitive Psychology, 7, 573-605.

Rumelhart, D. E., Hinton, G. E., \& Williams, R. J. (1986). Learning internal representations by error propagation. In D. E. Rumelhart \& J. L. McClelland (Eds.), Parallel distributed processing: Explorations in the microstructure of cognition: Vol 1. Foundations (pp.318362). Cambridge, MA: Bradford Books/MIT Press.

Schliemann, A. D., \& Carraher, D. W. (1993). Proportional reasoning in and out of school. In P. Light \& G. Butterworth (Eds.), Context and cognition: Ways of learning and knowing (pp. 47-73). Hillsdale, NJ: Erlbaum.

Shanks, D. R. (1992). Connectionist accounts of the inverse base-rate effect in categorization. Journal of Neural Computing, Artificial Intelligence \& Cognitive Research, 4, 3-18.

Shanks, D. R., Charles, D., Darby, R. J., \& Azmi, A. (1998). Configural processes in human associative learning. Journal of Experimental Psychology: Learning, Memory, and Cognition, 24, 1353-1378.

Shanks, D. R., Darby, R. J., \& Charles, D. (1998). Resistance to interference in human associative learning: evidence of configural processing. Journal of Experimental Psychology: Learning, Memory, and Cognition, 24, 136-150.

Shanks, D. R., \& Gluck, M. A. (1994). Tests of an adaptive network model for the identification and categorization of continuous-dimension stimuli. Connection Science, 6, 59-89.

Shepard, R. N., Hovland, C. L., \& Jenkins, H. M. (1961). Learning and memorization of classification. Psychological Monographs, 75(13, Whole No. 517).

Shin, H. J., \& Nosofsky, R. M. (1992). Similarity-scaling studies of dot-pattern classification and recognition. Journal of Experimental Psychology: General, 121, 278-304.

Smith, E. E., \& Medin, D. L. (1981). Categories and concepts. Cambridge: Harvard University press.

Smith, E. E., \& Solman, S. A. (1994). Similarity- versus rule-based categorization. Memory \& Cognition, 22, 377-386. 
Thomas, R. D. (1998). Learning correlations in categorization tasks using large, ill-defined categories. Journal of Experimental Psychology: Learning, Memory, and Cognition, 24, 119-143.

Vandierendonck, A. (1995). A parallel rule activation and rule synthesis model for generation in category learning. Psychonomic Bulletin \& Review, 2, 442-459.

Wasserman, E. A., \& Berglan, L. R. (1998). Backward blocking and recovery from overshadowing in human causal judgment: The role of within-compound associations. Quarterly Journal of Experimental Psychology, 51B, 121-138.

Wattenmaker, W. D. (1992). Relational properties and memory-based category representation. Journal of Experimental Psychology: Learning, Memory and Cognition, 18, 1125-1138.

Wattenmaker, W. D., Dewey, G. I., Murphy, T. D., \& Medin, D. L. (1986). Linear separability and concept learning: Context, relational properties, and concept naturalness. $\underline{\text { Cognitive }}$ Psychology, 18, 158-194.

Wickens, T. D. (1989). Multiway contingency tables analysis for the social sciences. Hillsdale, NJ: Erlbaum.

Williams, D. A., Sagness, K. E, \& McPhee, J. E. (1994). Configural and elemental strategies in predictive learning. Journal of Experimental Psychology: Learning, Memory, and Cognition, 20, 694-709.

Zaki, S. R., \& Nosofsky, R. M. (2001). Exemplar accounts of blending and distinctiveness effects in perceptual old-new recognition. Journal of Experimental Psychology: Learning, Memory, and Cognition, 27, 1022-1041. 
Table 2.1 Mean Probabilities of Category A Responses in Experiments 1 and 2 for Novel Transfer Items in Each Area and Test Context (Left or Right).

\begin{tabular}{lllll}
\hline & \multicolumn{4}{c}{ Area } \\
\cline { 2 - 4 } Condition & 1 & 2 & 3 & 4 \\
\hline
\end{tabular}

Experiment 1

Randomized-context

$\begin{array}{lllll}\text { Left } & .79 & .17 & .26 & .33 \\ \text { Right } & .79 & .20 & .22 & .27\end{array}$

Systematic-context

\begin{tabular}{lllll} 
Left & .79 & .09 & .07 & .44 \\
Right & .75 & .33 & .28 & .31 \\
\hline
\end{tabular}

Experiment 2

Systematic-context

\begin{tabular}{lllll} 
Left & .80 & .16 & .19 & .62 \\
Right & .74 & .47 & .39 & .40 \\
\hline
\end{tabular}


Table 2.2 Number and Percentage of Participants Identified as Using a Particular Strategy by k-Means Cluster Analysis in Experiment 1.

\begin{tabular}{|c|c|c|}
\hline \multirow[b]{3}{*}{ Cluster } & \multicolumn{2}{|c|}{ Test context } \\
\hline & Left & Right \\
\hline & $\mathrm{n} \quad \%$ & n $\%$ \\
\hline \multicolumn{3}{|c|}{ Randomized-context condition } \\
\hline True-boundary & 9 (29) & $7(23)$ \\
\hline Left-boundary & $8(26)$ & $7(23)$ \\
\hline Right-boundary & $3(10)$ & $5(16)$ \\
\hline$Y=300$ & $5(16)$ & $5(16)$ \\
\hline Unrecognized & $6(19)$ & $7(23)$ \\
\hline \multicolumn{3}{|c|}{ Systematic-context condition } \\
\hline True-boundary & $4(13)$ & $5(16)$ \\
\hline Left-boundary & $13(42)$ & $6(20)$ \\
\hline Right-boundary & $0(0)$ & $5(16)$ \\
\hline$Y=300$ & $6(16)$ & $5(16)$ \\
\hline Unrecognized & $8(26)$ & $10(32)$ \\
\hline
\end{tabular}


Table 2.3 Number and Percentage of Participants Identified as Using a Particular Strategy by k-Means Cluster Analysis in Experiment 2.

\begin{tabular}{|c|c|c|}
\hline \multirow[b]{3}{*}{ Cluster } & \multicolumn{2}{|c|}{ Test context } \\
\hline & Left & Right \\
\hline & $\mathrm{n} \quad \%$ & n $\%$ \\
\hline \multicolumn{3}{|c|}{ Systematic-context condition } \\
\hline True-boundary & $6(19)$ & $7(23)$ \\
\hline Left-boundary & $16(52)$ & $7(23)$ \\
\hline Right-boundary & $3(10)$ & $13(42)$ \\
\hline Unrecognized & $6(19)$ & $4(12)$ \\
\hline
\end{tabular}


Table 3.1 Mean Probabilities of Category A Responses in Experiment 3 for Novel Transfer Items in Each area and Test Context (Upper or Lower).

\begin{tabular}{|c|c|c|c|c|c|c|}
\hline \multirow[b]{2}{*}{ Group } & \multicolumn{3}{|c|}{ Randomized-context } & \multicolumn{3}{|c|}{ Systematic-context } \\
\hline & Area 1 & Area 2 & Area 3 & Area 1 & Area 2 & Area 3 \\
\hline \multicolumn{7}{|l|}{ Overall } \\
\hline Upper & .10 & .71 & .20 & .12 & .65 & .50 \\
\hline Lower & .12 & .70 & .22 & .41 & .66 & .14 \\
\hline \multicolumn{7}{|l|}{ True-boundary } \\
\hline Upper & & & & .05 & .72 & .19 \\
\hline Lower & & & & .05 & .58 & .06 \\
\hline \multicolumn{7}{|c|}{ Knowledge partitioning } \\
\hline Upper & & & & .14 & .74 & .88 \\
\hline Lower & & & & .92 & .78 & .12 \\
\hline
\end{tabular}


Table 3.2 Number and Percentage of Participants Identified as Using a Particular Strategy by k-Means Cluster Analysis in Experiment 3.

\begin{tabular}{|c|c|c|}
\hline \multirow[b]{3}{*}{ Cluster } & \multicolumn{2}{|c|}{ Test context } \\
\hline & Upper & Lower \\
\hline & n $\%$ & $\mathrm{n} \quad \%$ \\
\hline \multicolumn{3}{|c|}{ Randomized-context condition } \\
\hline True-boundary & $20(83)$ & 19(79) \\
\hline Upper-boundary & $3(13)$ & $4(17)$ \\
\hline Lower-boundary & $1(4)$ & $1(4)$ \\
\hline Unrecognized & $0(0)$ & $0(0)$ \\
\hline \multicolumn{3}{|c|}{ Systematic-context condition } \\
\hline True-boundary & $13(54)$ & $13(54)$ \\
\hline Upper-boundary & $11(46)$ & $0(0)$ \\
\hline Lower-boundary & $0(0)$ & $8(33)$ \\
\hline Unrecognized & $0(0)$ & $3(13)$ \\
\hline
\end{tabular}


Table 3.3 Mean Probabilities of Category A Responses in Experiment 4 for Old Transfer Items in Each Area and Test Context.

\begin{tabular}{lccc}
\hline & \multicolumn{3}{c}{ Area } \\
\cline { 2 - 4 } Test context & 1 & 2 & 3 \\
\hline Congruent & .08 & .75 & .24 \\
Incongruent & .39 & .76 & .69 \\
\hline
\end{tabular}


Table 3.4 Mean Probabilities of Category A Responses in Experiment 4 for Novel Transfer Items in Each Area and Test Context (Upper or Lower).

\begin{tabular}{lccc}
\hline Group & Area 1 & Area 2 & Area 3 \\
\hline Overall & & & \\
Upper & .11 & .70 & .56 \\
Lower & .35 & .66 & .13 \\
True-boundary & & & \\
Upper & .07 & .80 & .19 \\
Lower & .13 & .72 & .09 \\
Knowledge partitioning & & & \\
Upper & .18 & .70 & .92 \\
Lower & .82 & .76 & .19 \\
\hline
\end{tabular}


Table 3.5 Contingency Rating Results in Experiment 4 and Significance Tests for Deviation From Chance (.50)

\begin{tabular}{|c|c|c|c|c|c|c|c|c|c|c|}
\hline \multirow[b]{2}{*}{ Group } & \multicolumn{4}{|c|}{$\mathrm{X}$} & \multicolumn{4}{|c|}{$\mathrm{Y}$} & \multicolumn{2}{|c|}{ Context } \\
\hline & 100 & 300 & 500 & 700 & 200 & 400 & 600 & 800 & Upper & Lower \\
\hline Normative & .00 & .52 & .53 & .00 & .00 & .52 & .52 & .00 & .50 & .50 \\
\hline Overall & .47 & $.61^{*}$ & .48 & .47 & .59 & $.61^{*}$ & .37 & $.28 * *$ & .45 & .54 \\
\hline KP & .47 & .55 & .48 & .54 & .64 & .68 & .36 & $.20 *$ & .40 & .56 \\
\hline TB & .54 & .66 & .54 & .54 & .63 & .59 & .36 & .35 & .44 & .57 \\
\hline
\end{tabular}


Table 4.1 Mean Probability of Category A Responses in Experiment 1 for Training Items Presented At Transfer in Each Area and Test Context (Congruent or Incongruent).

\begin{tabular}{lccc}
\hline & \multicolumn{3}{c}{ Area } \\
\cline { 2 - 4 } Context & 1 & 2 & 3 \\
\hline \multicolumn{4}{c}{ Randomized-context condition } \\
Congruent & .29 & .82 & .46 \\
Incongruent & .28 & .81 & .54 \\
\hline \multicolumn{4}{c}{ Systematic-context condition } \\
Congruent & .29 & .80 & .37 \\
Incongruent & .47 & .75 & .57 \\
\hline
\end{tabular}


Table 4.2 Mean Probability of Category A Responses in Experiment 1 for Novel Transfer Items in Each Area and Test Context (Upper or Lower).

\begin{tabular}{|c|c|c|c|c|c|c|}
\hline \multirow[b]{3}{*}{ Participant group } & \multicolumn{6}{|c|}{ Condition } \\
\hline & \multicolumn{3}{|c|}{ Randomized-context } & \multicolumn{3}{|c|}{ Systematic-context } \\
\hline & Area 1 & Area 2 & Area 3 & Area 1 & Area 2 & Area 3 \\
\hline \multicolumn{7}{|l|}{ Overall } \\
\hline Upper & .27 & .67 & .28 & .25 & .69 & .50 \\
\hline Lower & .31 & .65 & .30 & .50 & .60 & .19 \\
\hline \multicolumn{7}{|l|}{ True-boundary } \\
\hline Upper & & & & .18 & .64 & .20 \\
\hline Lower & & & & .19 & .64 & .19 \\
\hline \multicolumn{7}{|c|}{ Knowledge partitioning } \\
\hline Upper & & & & .17 & .83 & .87 \\
\hline Lower & & & & .84 & .66 & .17 \\
\hline
\end{tabular}


Table 4.3 Number and Percentage of Participants Identified as Using a Particular Strategy by K-Means Cluster Analysis in Both Experiments.

\begin{tabular}{|c|c|c|}
\hline \multirow[b]{3}{*}{ Cluster } & \multicolumn{2}{|c|}{ Test context } \\
\hline & Upper & Lower \\
\hline & $\mathrm{n} \quad \%$ & n $\%$ \\
\hline \multicolumn{3}{|c|}{ Experiment 1} \\
\hline \multicolumn{3}{|c|}{ Randomized-context condition } \\
\hline True-boundary & 19 (79) & $15(63)$ \\
\hline Upper-boundary & $2(8)$ & $2(8)$ \\
\hline Lower-boundary & $2(8)$ & $3(12)$ \\
\hline Unrecognized & $1(5)$ & $4(17)$ \\
\hline \multicolumn{3}{|c|}{ Systematic-context condition } \\
\hline True-boundary & $11(46)$ & $12(50)$ \\
\hline Upper-boundary & $11(46)$ & $0 \quad(0)$ \\
\hline Lower-boundary & $2(8)$ & $12(50)$ \\
\hline Unrecognized & $0 \quad(0)$ & $0 \quad(0)$ \\
\hline \multicolumn{3}{|c|}{ Experiment 2} \\
\hline \multicolumn{3}{|c|}{ Systematic-context condition } \\
\hline True-boundary & $7(44)$ & $7(44)$ \\
\hline Upper-boundary & $8(50)$ & $1(6)$ \\
\hline Lower-boundary & $0 \quad(0)$ & $7(44)$ \\
\hline Unrecognized & $1(6)$ & $1(6)$ \\
\hline
\end{tabular}


Table 4.4 Mean Probability of Category A Responses in Experiment 2 for Training Items Present At Transfer in Each Area and Test Context (Congruent or Incongruent).

\begin{tabular}{lccc}
\hline & \multicolumn{3}{c}{ Area } \\
\cline { 2 - 4 } Context & 1 & 2 & 3 \\
\hline \multicolumn{4}{c}{ Systematic-context condition } \\
Congruent & .16 & .83 & .36 \\
Incongruent & .53 & .77 & .53 \\
\hline \multicolumn{4}{c}{ Upper-only condition } \\
Congruent & .24 & .76 \\
Incongruent & .34 & .74 \\
\hline \multicolumn{4}{c}{ Lower-only condition } \\
Congruent & .70 & .18 \\
Incongruent & .62 & .22 \\
\hline
\end{tabular}


Table 4.5 Mean Probability of Category A Responses in Experiment 2 for Novel Transfer Items in Each Area and Test Context (Upper or Lower).

\begin{tabular}{|c|c|c|c|c|c|c|c|c|c|}
\hline \multirow[b]{3}{*}{ Context } & \multicolumn{9}{|c|}{ Condition } \\
\hline & \multicolumn{3}{|c|}{ Systematic-context } & \multicolumn{3}{|c|}{ Upper-only } & \multicolumn{3}{|c|}{ Lower-only } \\
\hline & Area 1 & Area 2 & Area 3 & Area 1 & Area 2 & Area 3 & Area 1 & Area 2 & Area 3 \\
\hline Upper & .20 & .57 & .46 & .16 & .68 & .90 & .75 & .50 & .25 \\
\hline Lower & .51 & .60 & .19 & .31 & .64 & .78 & .84 & .60 & .14 \\
\hline
\end{tabular}


Table 4.6 Contingency Ratings in Experiment 2 and Significance Tests for Deviation From Chance (.50).

\begin{tabular}{|c|c|c|c|c|c|c|c|c|c|c|}
\hline & \multicolumn{4}{|c|}{$\mathrm{X}$} & \multicolumn{4}{|c|}{$\mathrm{Y}$} & \multicolumn{2}{|c|}{ Context } \\
\hline & 100 & 300 & 500 & 700 & 200 & 400 & 600 & 800 & Upper & Lower \\
\hline Normative & .00 & .52 & .53 & .00 & .00 & .52 & .52 & .00 & .50 & .50 \\
\hline Observed & $.28 *$ & $.76^{* *}$ & .55 & $.21 * *$ & $.33 *$ & $.68^{*}$ & .59 & $.42 *$ & .45 & .57 \\
\hline $\mathrm{KP}$ & .54 & .65 & .42 & $.19 *$ & .45 & .68 & $.66^{*}$ & .54 & .45 & .47 \\
\hline TB & $.00 * *$ & $.94 * *$ & $.76^{*}$ & $.06 * *$ & $.09 *$ & .72 & .46 & $.12^{*}$ & .44 & .57 \\
\hline
\end{tabular}

Note: KP: knowledge partitioning group and TB: true-boundary group 
Table 6.1 Observed and Predicted Proportion Correct at Learning in Conceptual Category Learning Tasks.

\begin{tabular}{|c|c|c|c|c|c|c|c|c|c|c|c|}
\hline \multirow[b]{2}{*}{ Participant group } & \multirow[b]{2}{*}{$\mathrm{n}$} & \multicolumn{10}{|c|}{ Training block } \\
\hline & & 1 & 2 & 3 & 4 & 5 & 6 & 7 & 8 & 9 & 10 \\
\hline & \multicolumn{11}{|c|}{ Randomized-context condition (Experiment 1) } \\
\hline \multicolumn{12}{|l|}{ Overall } \\
\hline Observed & 23 & .53 & .59 & .65 & .67 & .71 & .73 & .76 & .80 & .82 & .84 \\
\hline ALCOVE & & .52 & .58 & .62 & .66 & .68 & .71 & .76 & .80 & .80 & .81 \\
\hline \multirow[t]{2}{*}{ ATRIUM } & & .51 & .58 & .62 & .66 & .68 & .71 & .74 & .77 & .79 & .80 \\
\hline & \multicolumn{11}{|c|}{ Systematic-context condition (Experiment 2) } \\
\hline \multicolumn{12}{|l|}{ KP } \\
\hline Observed & 4 & .58 & .74 & .78 & .79 & .87 & .88 & .89 & .91 & .95 & .95 \\
\hline ALCOVE & & .53 & .65 & .75 & .80 & .83 & .85 & .87 & .89 & .90 & .90 \\
\hline ATRIUM & & .57 & .64 & .74 & .82 & .84 & .86 & .87 & .88 & .88 & .90 \\
\hline \multicolumn{12}{|l|}{ TB } \\
\hline Observed & 3 & .70 & .76 & .78 & .79 & .80 & .78 & .77 & .72 & .80 & .82 \\
\hline ALCOVE & & .49 & .54 & .62 & .68 & .72 & .74 & .76 & .78 & .80 & .81 \\
\hline ATRIUM & & .59 & .69 & .73 & .75 & .75 & .78 & .77 & .78 & .79 & .79 \\
\hline ote. $\mathrm{TB}=$ partici & & $\mathrm{u}$ & $\mathrm{rm}$ & $\mathrm{ppl}$ & the & ue bc & indal & in $b$ & h co & exts; & \\
\hline
\end{tabular}


Table 6.2 Best Fitting Parameter Values for ALCOVE when Fit to Conceptual Category Learning Tasks and Fit Statistics.

\begin{tabular}{|c|c|c|c|}
\hline \multirow[b]{3}{*}{ Parameter } & \multicolumn{3}{|c|}{ Condition } \\
\hline & \multirow{2}{*}{$\begin{array}{c}\text { Randomized-context } \\
\text { Overall }\end{array}$} & \multicolumn{2}{|c|}{ Systematic-context } \\
\hline & & TB & KP \\
\hline \multicolumn{4}{|c|}{ Training } \\
\hline $\mathrm{C}$ & 9.90 & 5.53 & 9.89 \\
\hline$\phi$ & 0.60 & 1.98 & 1.47 \\
\hline$\lambda_{\mathrm{w}}$ & 0.07 & 0.01 & 0.03 \\
\hline$\lambda_{\alpha}$ & 0.01 & 0.06 & 0.01 \\
\hline$-2 \log L$ & 1877.11 & 856.79 & 816.47 \\
\hline \multicolumn{4}{|c|}{ Transfer } \\
\hline $\mathrm{C}$ & 3.97 & 2.03 & 3.68 \\
\hline$\phi$ & 2.23 & 3.06 & 1.59 \\
\hline$\lambda_{\mathrm{w}}$ & 0.02 & 0.01 & 0.03 \\
\hline$\lambda_{\alpha}$ & 0.09 & 0.01 & 0.02 \\
\hline$-2 \log L$ & 180.36 & 50.96 & 287.12 \\
\hline
\end{tabular}

Note. TB = participants who uniformly applied the true boundary in both contexts; KP = participants who showed complete partitioning by using the appropriate partial boundary in each of the two contexts. 
Table 6.3 Observed and Predicted Proportion Correct at Learning in Experiment 5.

\begin{tabular}{lcccccccccc}
\hline & \multicolumn{10}{c}{ Training block } \\
\cline { 3 - 10 } Participant group & $\mathrm{n}$ & 1 & 2 & 3 & 4 & 5 & 6 & 7 & 8 \\
\hline & & \multicolumn{10}{c}{ Randomized-context condition } \\
Overall & & & & & & & & & \\
Observed & 18 & .52 & .59 & .63 & .67 & .69 & .68 & .75 & .76 \\
ALCOVE & & .52 & .54 & .60 & .62 & .66 & .69 & .72 & .73 \\
ATRIUM & & .50 & .54 & .62 & .66 & .67 & .70 & .71 & .71 \\
\hline & & & Systematic-context condition & & \\
KP & & & & & & & & & \\
Observed & 9 & .54 & .66 & .67 & .71 & .74 & .79 & .80 & .84 \\
ALCOVE & & .49 & .56 & .60 & .69 & .74 & .78 & .81 & .83 \\
ATRIUM & & .53 & .64 & .69 & .73 & .74 & .75 & .77 & .77 \\
TB & & & & & & & & & \\
Observed & 10 & .55 & .64 & .67 & .71 & .74 & .80 & .80 & .82 \\
ALCOVE & & .49 & .54 & .59 & .66 & .72 & .76 & .79 & .82 \\
ATRIUM & & .53 & .66 & .68 & .72 & .74 & .75 & .76 & .77 \\
\hline
\end{tabular}

Note. TB = participants who uniformly applied the true boundary in both contexts;

$\mathrm{KP}=$ participants who showed complete partitioning by using the appropriate partial boundary in each of the two contexts. 
Table 6.4 Best Fitting Parameter Values for ALCOVE when Fit to Perceptual Category Learning Tasks and Fit Statistics.

\begin{tabular}{|c|c|c|c|}
\hline \multirow[b]{3}{*}{ Parameter } & \multicolumn{3}{|c|}{ Condition } \\
\hline & \multirow{2}{*}{$\begin{array}{c}\text { Randomized-context } \\
\text { Overall }\end{array}$} & \multicolumn{2}{|c|}{ Systematic-context } \\
\hline & & TB & KP \\
\hline \multicolumn{4}{|c|}{ Training } \\
\hline $\mathrm{C}$ & 9.90 & \multicolumn{2}{|c|}{9.90} \\
\hline$\phi$ & 2.10 & \multicolumn{2}{|c|}{1.92} \\
\hline$\lambda_{\mathrm{w}}$ & 0.01 & \multicolumn{2}{|c|}{0.01} \\
\hline$\lambda_{\alpha}$ & 0.08 & \multicolumn{2}{|c|}{0.03} \\
\hline$-2 \log L$ & 1553.28 & 1139.96 & 1204.09 \\
\hline \multicolumn{4}{|c|}{ Transfer } \\
\hline $\mathrm{C}$ & 9.11 & \multicolumn{2}{|c|}{9.89} \\
\hline$\phi$ & 2.15 & \multicolumn{2}{|c|}{6.09} \\
\hline$\lambda_{\mathrm{w}}$ & 0.01 & 0.06 & 0.01 \\
\hline$\lambda_{\alpha}$ & 0.07 & 0.01 & 0.15 \\
\hline$-2 \log L$ & 128.64 & 76.67 & 213.11 \\
\hline \multicolumn{4}{|c|}{$\begin{array}{l}\text { Note. } \mathrm{TB} \text { = participants who uniformly applied the true boundary in } \\
\text { both contexts; KP = participants who showed complete partitioning }\end{array}$} \\
\hline
\end{tabular}


Table 6.5 Best Fitting Parameter Values for ATRIUM when Fit to Conceptual Category Learning Tasks and Fit Statistics.

\begin{tabular}{|c|c|c|c|}
\hline \multirow[b]{3}{*}{ Parameter } & \multicolumn{3}{|c|}{ Condition } \\
\hline & \multirow{2}{*}{$\begin{array}{c}\text { Randomized-context } \\
\text { Overall } \\
\end{array}$} & \multicolumn{2}{|c|}{ Systematic-context } \\
\hline & & TB & $\mathrm{KP}$ \\
\hline \multicolumn{4}{|c|}{ Training } \\
\hline $\mathrm{C}$ & 9.89 & 6.79 & 9.75 \\
\hline$\phi$ & 1.31 & 0.78 & 1.93 \\
\hline$\phi_{g}$ & 0.92 & 1.26 & 0.50 \\
\hline$\gamma_{r}$ & 6.63 & 6.23 & 9.80 \\
\hline$\lambda_{e}$ & 0.25 & 0.27 & 1.51 \\
\hline$\lambda_{r 1}$ & 1.74 & 1.99 & 1.98 \\
\hline$\lambda_{r 2}$ & 0.56 & 1.83 & 0.51 \\
\hline$\lambda_{g}$ & 0.40 & 1.00 & 0.23 \\
\hline$\lambda_{\alpha}$ & 0.02 & 0.64 & 0.05 \\
\hline$-2 \log L$ & 1986.97 & 876.18 & 831.32 \\
\hline \multicolumn{4}{|c|}{ Transfer } \\
\hline $\mathrm{C}$ & 9.88 & 7.01 & 9.44 \\
\hline$\phi$ & 3.95 & 9.79 & 7.71 \\
\hline$\phi_{g}$ & 0.60 & 0.30 & 0.45 \\
\hline$\gamma_{r}$ & 6.45 & 0.27 & 4.72 \\
\hline$\lambda_{e}$ & 0.04 & 0.06 & 0.55 \\
\hline$\lambda_{r 1}$ & 1.98 & 1.25 & 1.86 \\
\hline$\lambda_{r 2}$ & 0.12 & 1.14 & 0.01 \\
\hline$\lambda_{g}$ & 0.66 & 1.34 & 0.80 \\
\hline$\lambda_{\alpha}$ & 0.01 & 0.58 & 0.14 \\
\hline$-2 \log L$ & 134.69 & 49.50 & 52.77 \\
\hline \multicolumn{4}{|c|}{$\begin{array}{l}\text { Note. TB = participants who uniformly applied the true boundary ir } \\
\text { both contexts; KP = participants who showed complete partitioning }\end{array}$} \\
\hline
\end{tabular}


Table 6.6 The AIC measure for ALCOVE and ATRIUM.

\begin{tabular}{|c|c|c|c|c|}
\hline \multirow[b]{2}{*}{ Condition } & \multicolumn{2}{|c|}{ Fit to the training data } & \multicolumn{2}{|c|}{ Fit to the transfer data } \\
\hline & ALCOVE & ATRIUM & ALCOVE & ATRIUM \\
\hline Experiment 1 & & & & \\
\hline Randomized-Context & $1885.11(4)^{\mathrm{a}}$ & $2004.97 \quad(9)$ & 188.36 & 152.69 \\
\hline \multicolumn{5}{|l|}{ Experiment 2} \\
\hline Systematic-Context & 1689.26 & 1743.5 & 354.08 & 138.27 \\
\hline \multicolumn{5}{|l|}{ Experiment 5} \\
\hline Randomized-Context & 1561.28 & 1558.29 & 136.64 & $83.51 \quad(9)$ \\
\hline \multicolumn{5}{|l|}{ Experiment 5} \\
\hline Systematic-Context & 2352.05 & $2265.98 \quad(9)$ & 301.78 & 169.64 \\
\hline
\end{tabular}

${ }^{\mathrm{a}}$ Number of free parameters used for modeling. 
Table 6.7 Best Fitting Parameter Values For ATRIUM when Fit to Perceptual Category Learning Tasks and Fit statistics.

\begin{tabular}{|c|c|c|c|}
\hline \multirow[b]{3}{*}{ Parameter } & \multicolumn{3}{|c|}{ Condition } \\
\hline & \multirow{2}{*}{$\begin{array}{c}\text { Randomized-context } \\
\text { Overall } \\
\end{array}$} & \multicolumn{2}{|c|}{ Systematic-context } \\
\hline & & TB & $\mathrm{KP}$ \\
\hline \multicolumn{4}{|c|}{ Training } \\
\hline $\mathrm{C}$ & 8.33 & \multicolumn{2}{|c|}{8.47} \\
\hline$\phi$ & 0.67 & \multicolumn{2}{|c|}{1.04} \\
\hline$\phi_{g}$ & 1.08 & \multicolumn{2}{|c|}{1.00} \\
\hline$\gamma_{r}$ & 8.85 & \multicolumn{2}{|c|}{8.38} \\
\hline$\lambda_{e}$ & 0.02 & \multicolumn{2}{|c|}{1.76} \\
\hline$\lambda_{r 1}$ & 1.21 & \multicolumn{2}{|c|}{0.99} \\
\hline$\lambda_{r 2}$ & 1.96 & \multicolumn{2}{|c|}{0.86} \\
\hline$\lambda_{g}$ & 0.35 & \multicolumn{2}{|c|}{0.67} \\
\hline$\lambda_{\alpha}$ & 0.95 & \multicolumn{2}{|c|}{0.01} \\
\hline$-2 \log L$ & 1540.29 & 1125.27 & 1122.71 \\
\hline \multicolumn{4}{|c|}{ Transfer } \\
\hline $\mathrm{C}$ & 5.10 & \multicolumn{2}{|c|}{9.10} \\
\hline$\phi$ & 2.52 & 4.29 & 3.68 \\
\hline$\phi_{g}$ & 0.27 & 0.31 & 0.59 \\
\hline$\gamma_{r}$ & 6.69 & \multicolumn{2}{|c|}{3.79} \\
\hline$\lambda_{e}$ & 0.55 & \multicolumn{2}{|c|}{0.47} \\
\hline$\lambda_{r 1}$ & 0.06 & \multicolumn{2}{|c|}{0.28} \\
\hline$\lambda_{r 2}$ & 1.94 & \multicolumn{2}{|c|}{0.20} \\
\hline$\lambda_{g}$ & 0.99 & 1.89 & 0.22 \\
\hline$\lambda_{\alpha}$ & 0.58 & 0.23 & 0.83 \\
\hline$-2 \log L$ & 65.51 & 77.96 & 65.68 \\
\hline \multicolumn{4}{|c|}{$\begin{array}{l}\text { Note. } \mathrm{TB}=\text { participants who uniformly applied the true boundary in } \\
\text { both contexts; KP = participants who showed complete partitioning by }\end{array}$} \\
\hline
\end{tabular}


Table 6.8 Averaged Gain for every Module in ATRIUM After Fitting the Groups of Participants in the Systematic-Context Condition in Experiment 5.

\begin{tabular}{cccccccc}
\hline & \multicolumn{3}{c}{ Upper context } & & \multicolumn{3}{c}{ Lower context } \\
\cline { 2 - 4 } Area & Exemplar & Rule 1 & Rule 2 & & Exemplar & Rule 1 & Rule 2 \\
\hline & 0.03 & 0.91 & 0.06 & & 0.05 & 0.12 & 0.83 \\
2 & 0.04 & 0.89 & 0.07 & & 0.03 & 0.08 & 0.89 \\
3 & 0.06 & 0.83 & 0.11 & & 0.02 & 0.07 & 0.91 \\
\hline & 0.15 & 0.74 & 0.11 & & 0.17 & 0.70 & 0.13 \\
1 & 0.22 & 0.46 & 0.32 & & 0.23 & 0.40 & 0.37 \\
3 & 0.23 & 0.16 & 0.61 & & 0.22 & 0.14 & 0.65 \\
\hline
\end{tabular}


Table 6.9 The goodness-of-fit (-2logL) between the ATRIUM prediction and the transfer data from the knowledge partitioning group with different choosing probability of both rule modules in both contexts.

\begin{tabular}{|c|c|c|c|c|c|c|c|c|c|c|c|}
\hline \multirow{2}{*}{$\begin{array}{c}\text { Upper } \\
\text { Context }\end{array}$} & \multicolumn{11}{|c|}{ Lower Context } \\
\hline & 0.0 & 0.1 & 0.2 & 0.3 & 0.4 & 0.5 & 0.6 & 0.7 & 0.8 & 0.9 & 1.0 \\
\hline 1.0 & 124.35 & 105.76 & 121.89 & 144.94 & 173.97 & 209.63 & 253.70 & 309.58 & 384.06 & 493.34 & 695.97 \\
\hline 0.9 & 112.53 & 93.94 & 110.06 & 133.12 & 162.14 & 197.80 & 241.87 & 297.75 & 372.24 & 481.52 & 684.14 \\
\hline 0.8 & 119.70 & 101.11 & 117.23 & 140.28 & 169.31 & 204.97 & 249.04 & 304.92 & 379.41 & 488.68 & 691.31 \\
\hline 0.7 & 136.08 & 117.49 & 133.61 & 156.66 & 185.69 & 221.35 & 265.42 & 321.30 & 395.79 & 505.06 & 707.69 \\
\hline 0.6 & 159.73 & 141.15 & 157.27 & 180.32 & 209.35 & 245.01 & 289.08 & 344.96 & 419.45 & 528.72 & 731.35 \\
\hline 0.5 & 190.84 & 172.25 & 188.37 & 211.42 & 240.45 & 276.11 & 320.18 & 376.06 & 450.55 & 559.82 & 762.45 \\
\hline 0.4 & 230.97 & 212.38 & 228.50 & 251.56 & 280.58 & 316.24 & 360.31 & 416.19 & 490.68 & 599.96 & 802.58 \\
\hline 0.3 & 283.70 & 265.11 & 281.23 & 304.29 & 333.31 & 368.97 & 413.04 & 468.93 & 543.41 & 652.69 & 855.31 \\
\hline 0.2 & 357.04 & 338.45 & 354.57 & 377.62 & 406.65 & 442.31 & 486.38 & 542.26 & 616.75 & 726.02 & 928.65 \\
\hline 0.1 & 474.45 & 455.87 & 471.99 & 495.04 & 524.07 & 559.73 & 603.80 & 659.68 & 734.17 & 843.44 & 1046.07 \\
\hline 0.0 & 1032.76 & 1014.18 & 1030.30 & 1053.35 & 1082.38 & 1118.04 & 1162.11 & 1217.99 & 1292.48 & 1401.75 & 1604.38 \\
\hline
\end{tabular}


Table 6.10 The goodness-of-fit (-2logL) between the ATRIUM prediction and the transfer data from the randomized-context condition with different choosing probability of both rule modules in both contexts.

\begin{tabular}{|c|c|c|c|c|c|c|c|c|c|c|c|}
\hline \multirow{2}{*}{$\begin{array}{l}\text { Upper } \\
\text { Context }\end{array}$} & \multicolumn{11}{|c|}{ Lower Context } \\
\hline & 0.0 & 0.1 & 0.2 & 0.3 & 0.4 & 0.5 & 0.6 & 0.7 & 0.8 & 0.9 & 1.0 \\
\hline 1.0 & 449.43 & 382.04 & 372.90 & 397.08 & 447.17 & 522.01 & 624.55 & 762.61 & 952.80 & 1234.15 & 1733.20 \\
\hline 0.9 & 279.51 & 212.44 & 203.63 & 228.17 & 278.62 & 353.83 & 456.78 & 595.25 & 785.87 & 1067.68 & 1567.20 \\
\hline 0.8 & 241.40 & 174.68 & 166.23 & 191.15 & 242.00 & 317.63 & 421.01 & 559.93 & 751.04 & 1033.36 & 1533.42 \\
\hline 0.7 & 257.58 & 191.25 & 183.20 & 208.53 & 259.82 & 335.91 & 439.77 & 579.21 & 770.85 & 1053.74 & 1554.39 \\
\hline 0.6 & 311.09 & 245.18 & 237.57 & 263.36 & 315.13 & 391.74 & 496.14 & 636.14 & 828.39 & 1111.92 & 1613.25 \\
\hline 0.5 & 399.01 & 333.56 & 326.43 & 352.74 & 405.05 & 482.22 & 587.23 & 727.88 & 920.81 & 1205.06 & 1707.18 \\
\hline 0.4 & 526.21 & 461.27 & 454.69 & 481.57 & 534.49 & 612.31 & 718.00 & 859.38 & 1053.09 & 1338.18 & 1841.20 \\
\hline 0.3 & 707.33 & 642.97 & 637.00 & 664.53 & 718.13 & 796.69 & 903.17 & 1045.39 & 1240.00 & 1526.06 & 2030.13 \\
\hline 0.2 & 979.01 & 915.30 & 910.02 & 938.28 & 992.68 & 1072.08 & 1179.47 & 1322.66 & 1518.33 & 1805.54 & 2310.86 \\
\hline 0.1 & 1461.00 & 1398.03 & 1393.55 & 1422.66 & 1477.97 & 1558.35 & 1666.80 & 1811.15 & 2008.08 & 2296.66 & 2803.48 \\
\hline 0.0 & 4594.40 & 4532.29 & 4528.72 & 4558.82 & 4615.20 & 4696.74 & 4806.45 & 4952.18 & 5150.63 & 5440.88 & 5949.56 \\
\hline
\end{tabular}




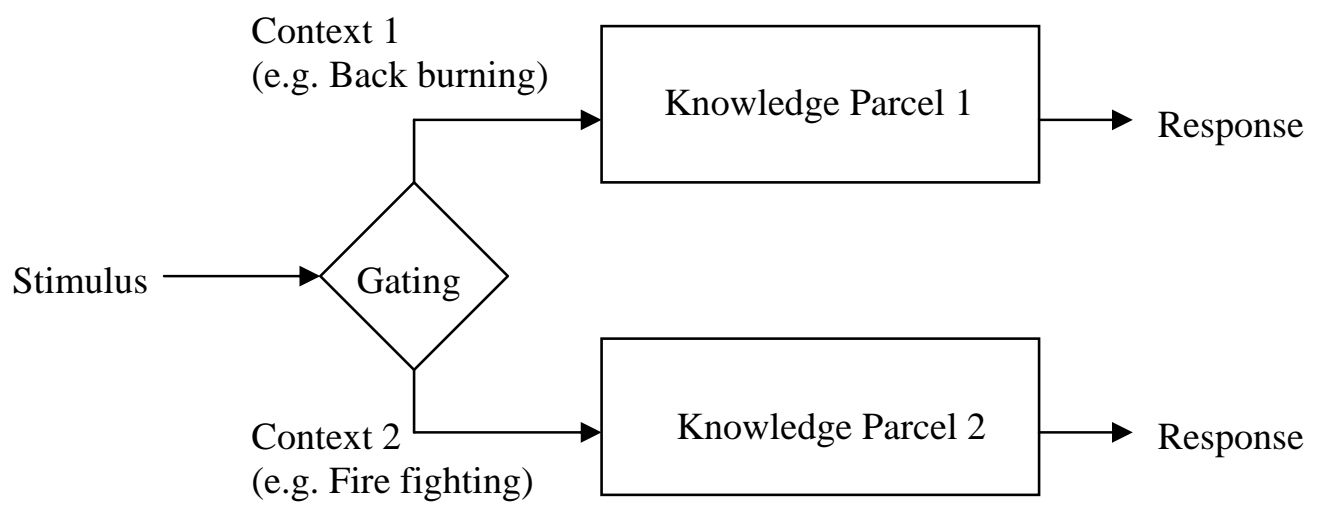

Figure 1.1 The schematic illustration of knowledge partitioning. Knowledge parcels are chosen for response according to context. 


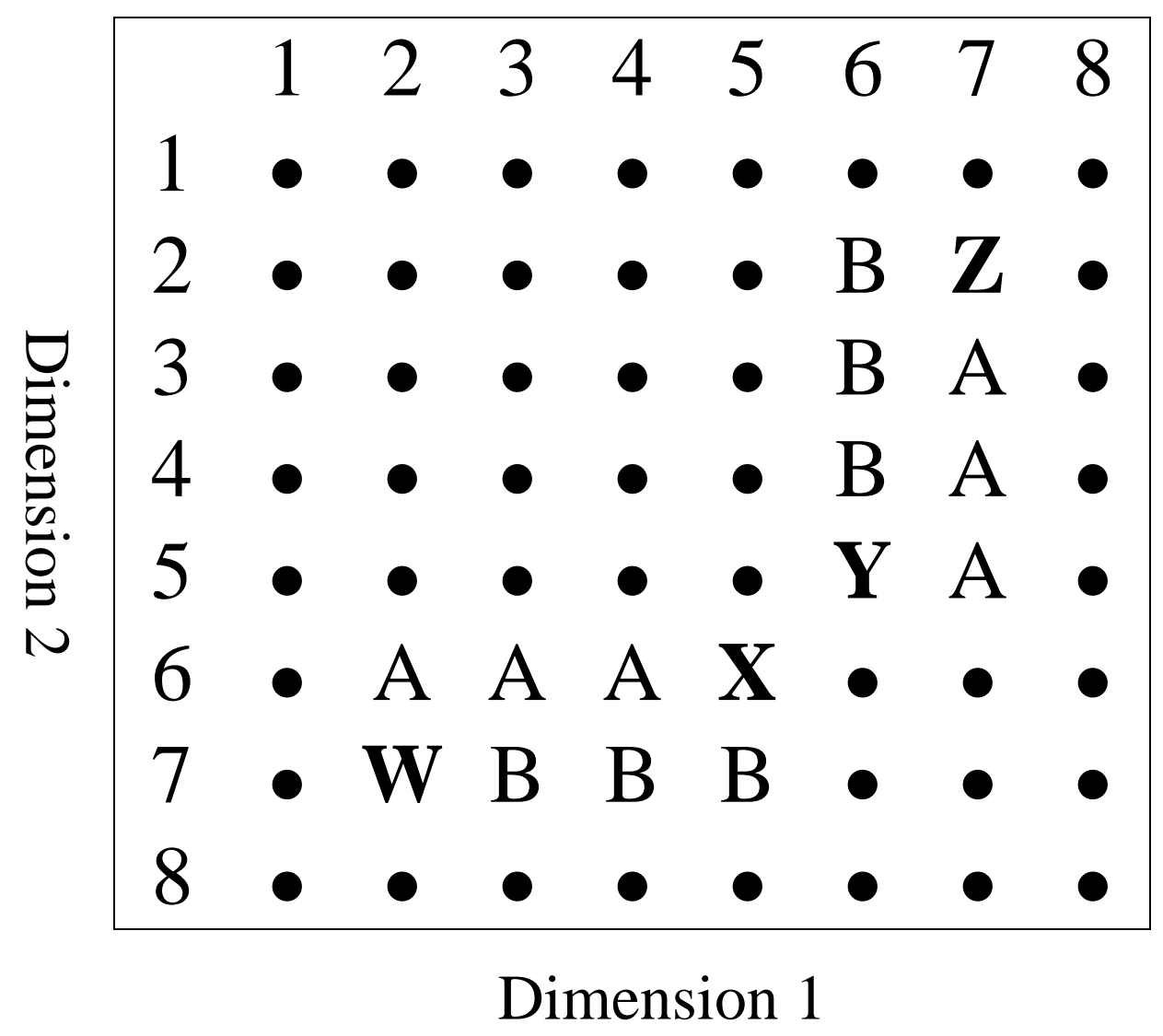

Figure 2.1 Stimulus space used by Aha and Goldstone (1992). The abscissa denotes the size of the rectangle and the ordinate denotes the position of a vertical line segment within it. The training instances are shown as $A$ and $B$. The four critical transfer items are marked with one of $\{W, X, Y, Z\}$. 


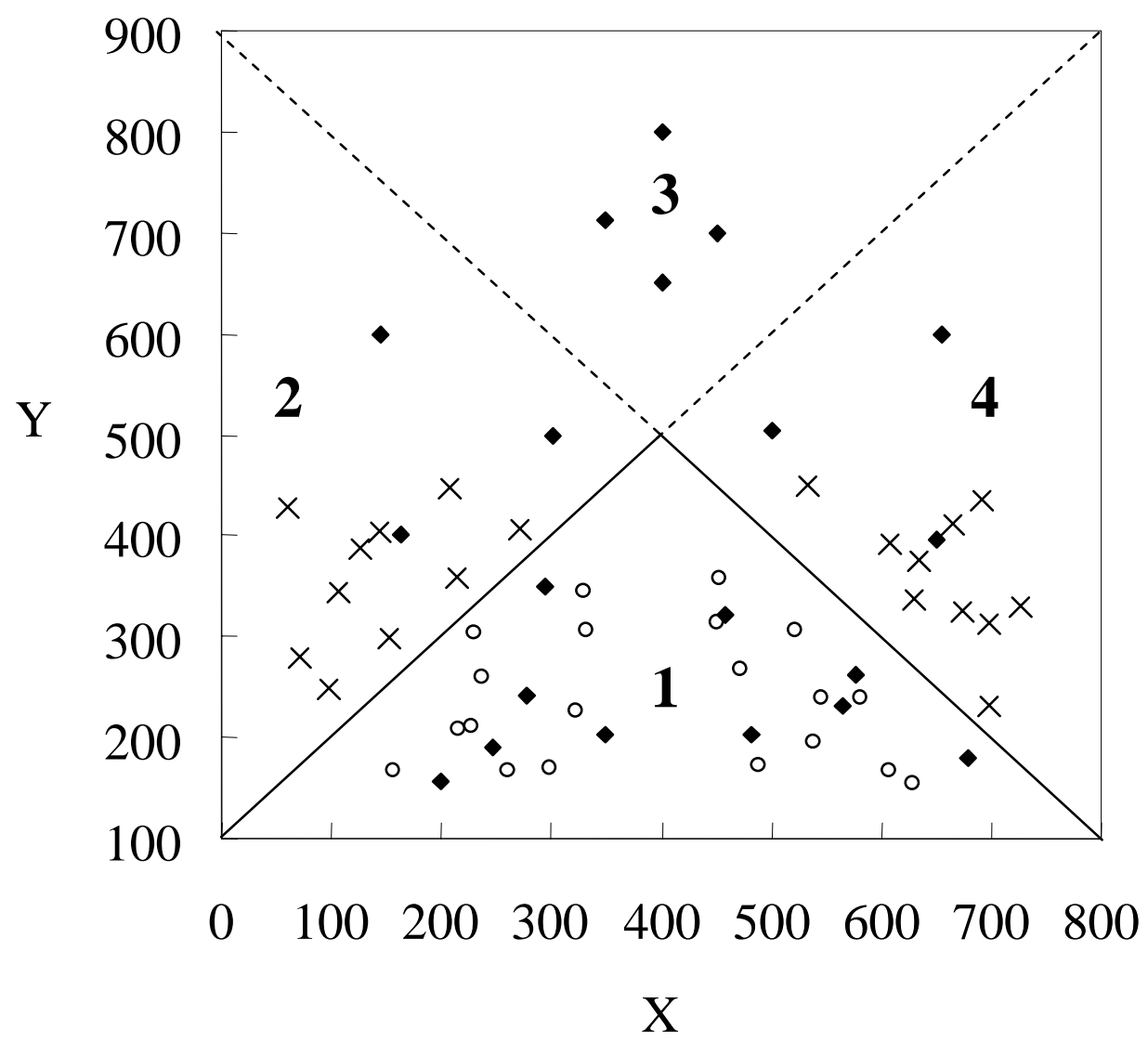

Figure 2.2 Stimulus space used in Experiment 1. The category boundary is indicated by the solid line. All stimuli above the boundary belong to category B, whereas all stimuli below belong to category A. The training instances are shown as open circles (category A) and crosses (category B). Note that $\mathrm{Y}<500$ for all training instances. Transfer items are shown as filled diamonds. See text for explanation of numbered areas. 

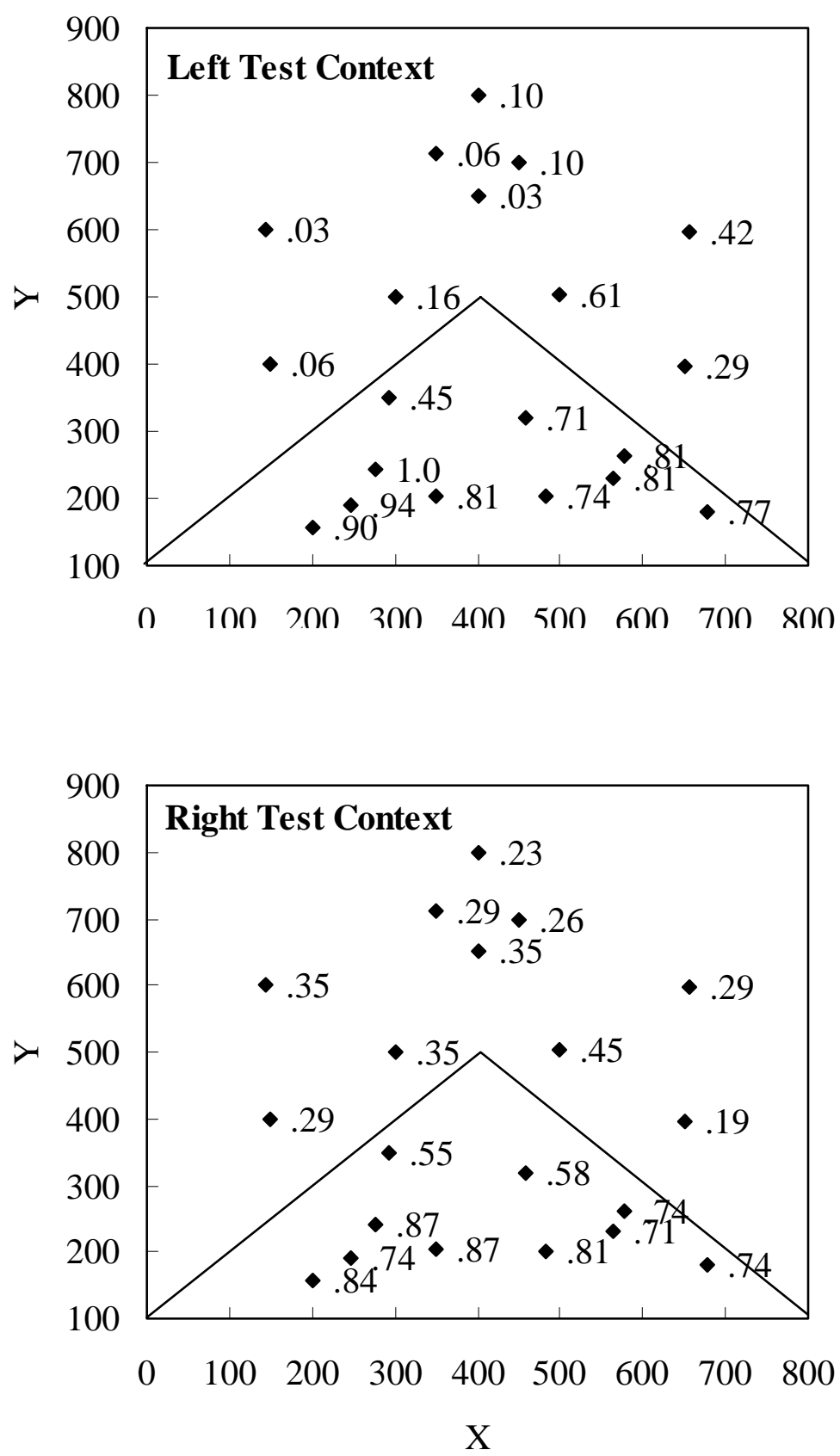

Figure 2.3 The average probability of category A responses for each transfer item in the systematic-context condition of Experiment 1. Top panel shows the "left" test context; the bottom panel shows the "right” context. 


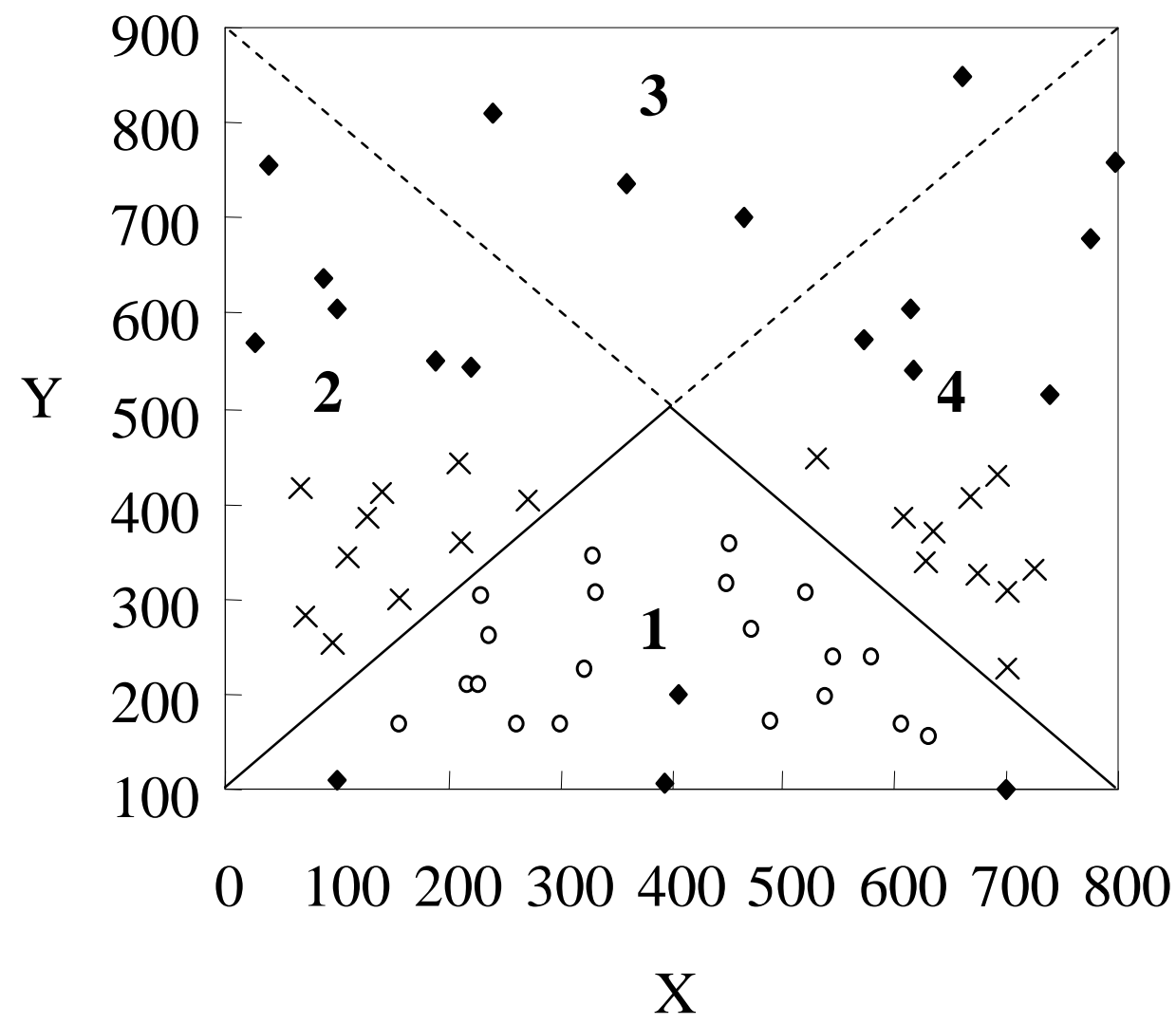

Figure 2.4 The stimulus space used in Experiment 2. The training instances are shown as open circles (category A) and crosses (category B). The transfer items are shown as filled diamonds. Numbered areas are as in Experiment 1. 

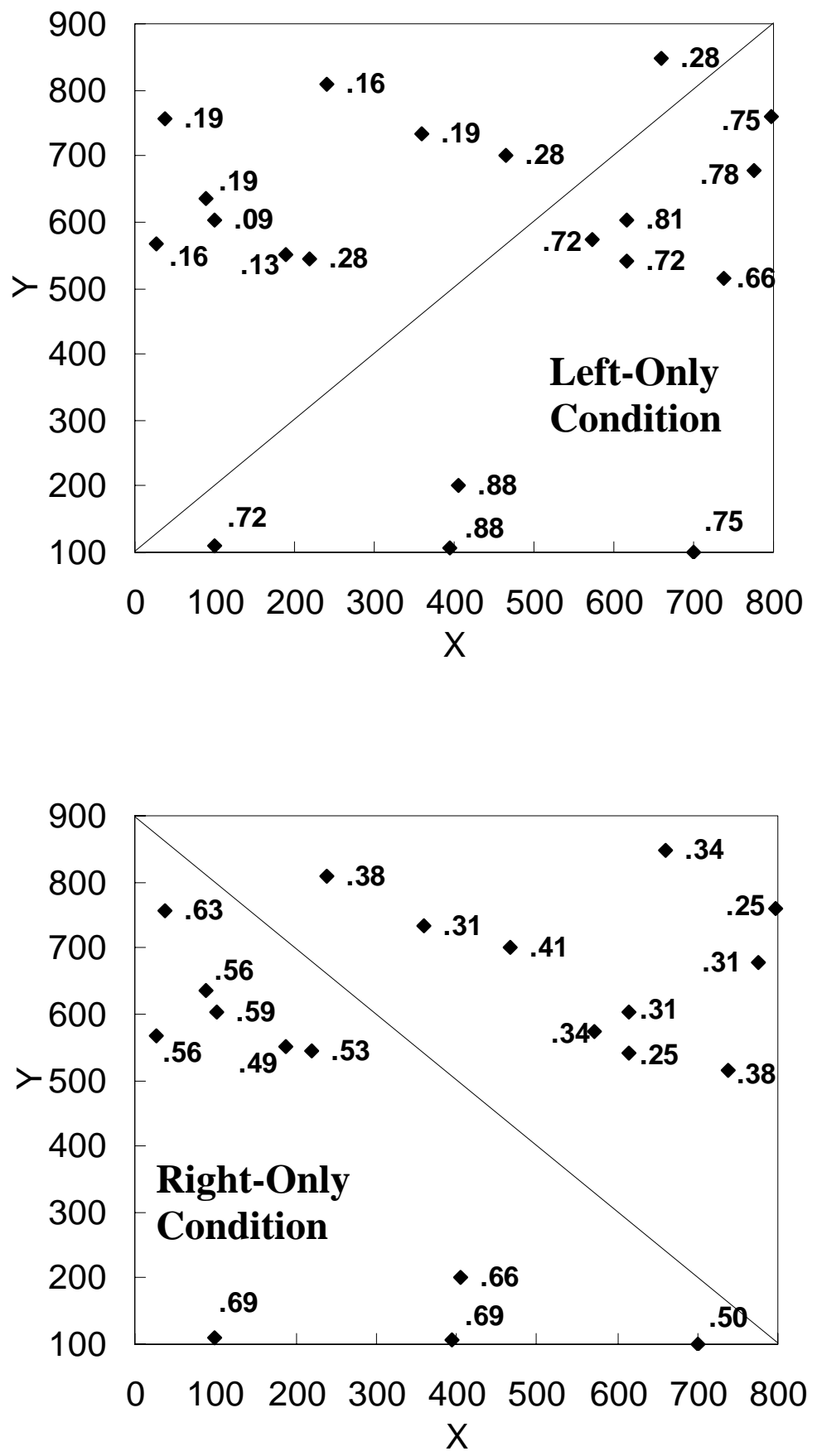

Figure 2.5 Average probabilities of category A responses for each transfer item in the two single-context conditions (left-only in top panel and right-only at bottom) in Experiment 2. 


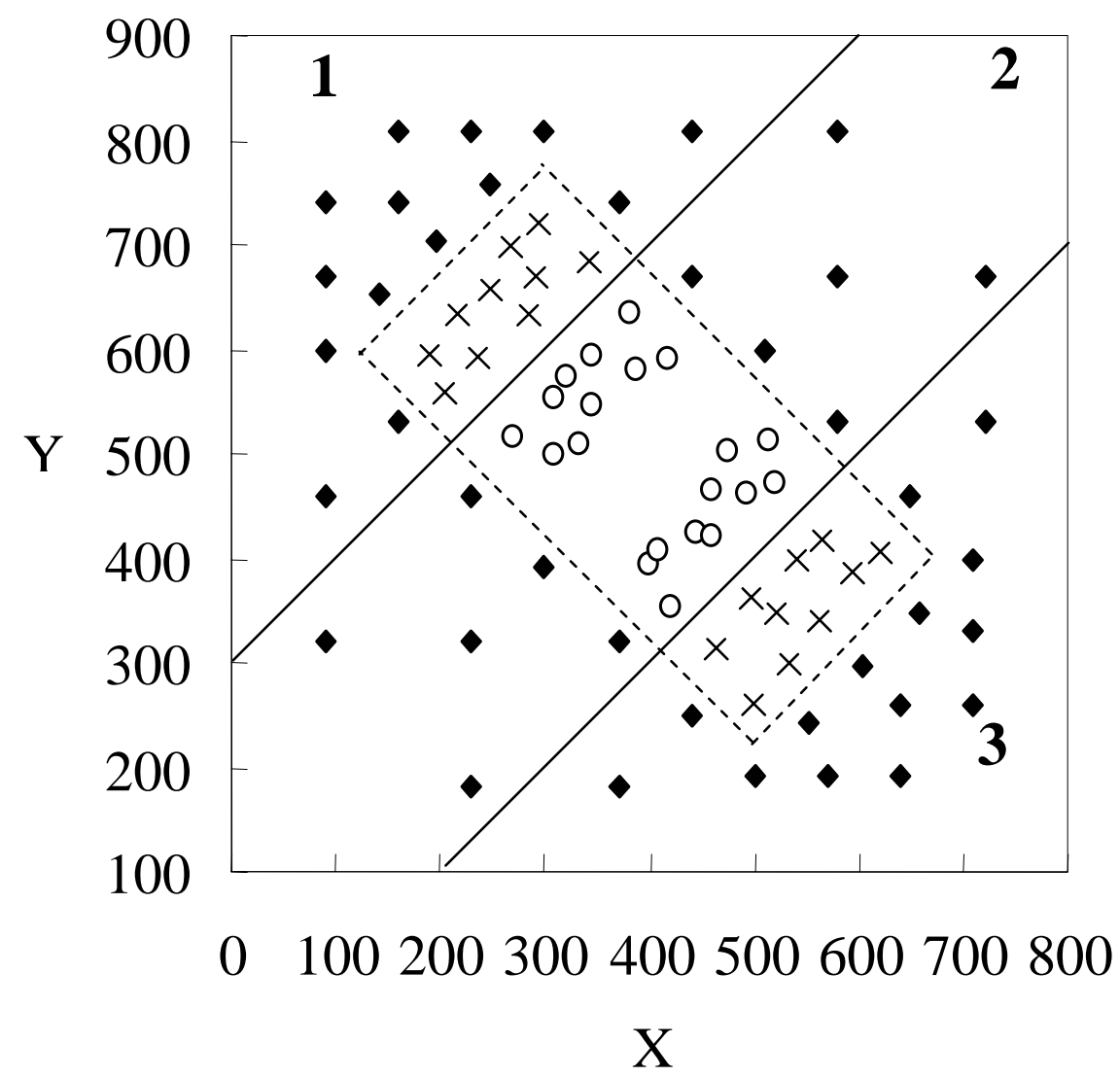

Figure 3.1 Stimulus space used in Experiments 3 and 4. Training instances were randomly chosen for each participant from within the dotted rectangle. Open circles and crosses show a representative set of training items belonging to category A and B, respectively. The filled diamonds represent common transfer items. The two solid lines represent the true category boundaries. See text for numbering of areas. 


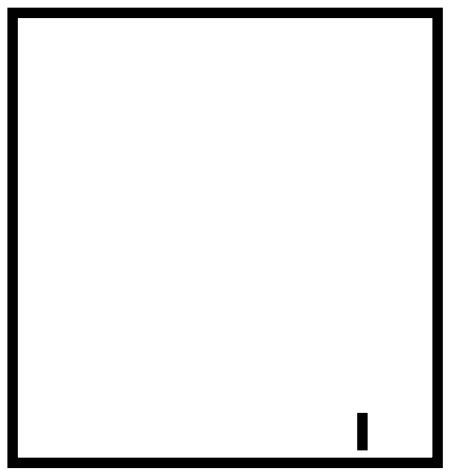

Figure 4.1 The stimuli used in Experiment 5 and 6 varied along two dimensions: the height of the rectangle and the position of a line segment in that rectangle. The context cue was instantiated by the color (i.e., red or green) of the rectangle and line segment. 

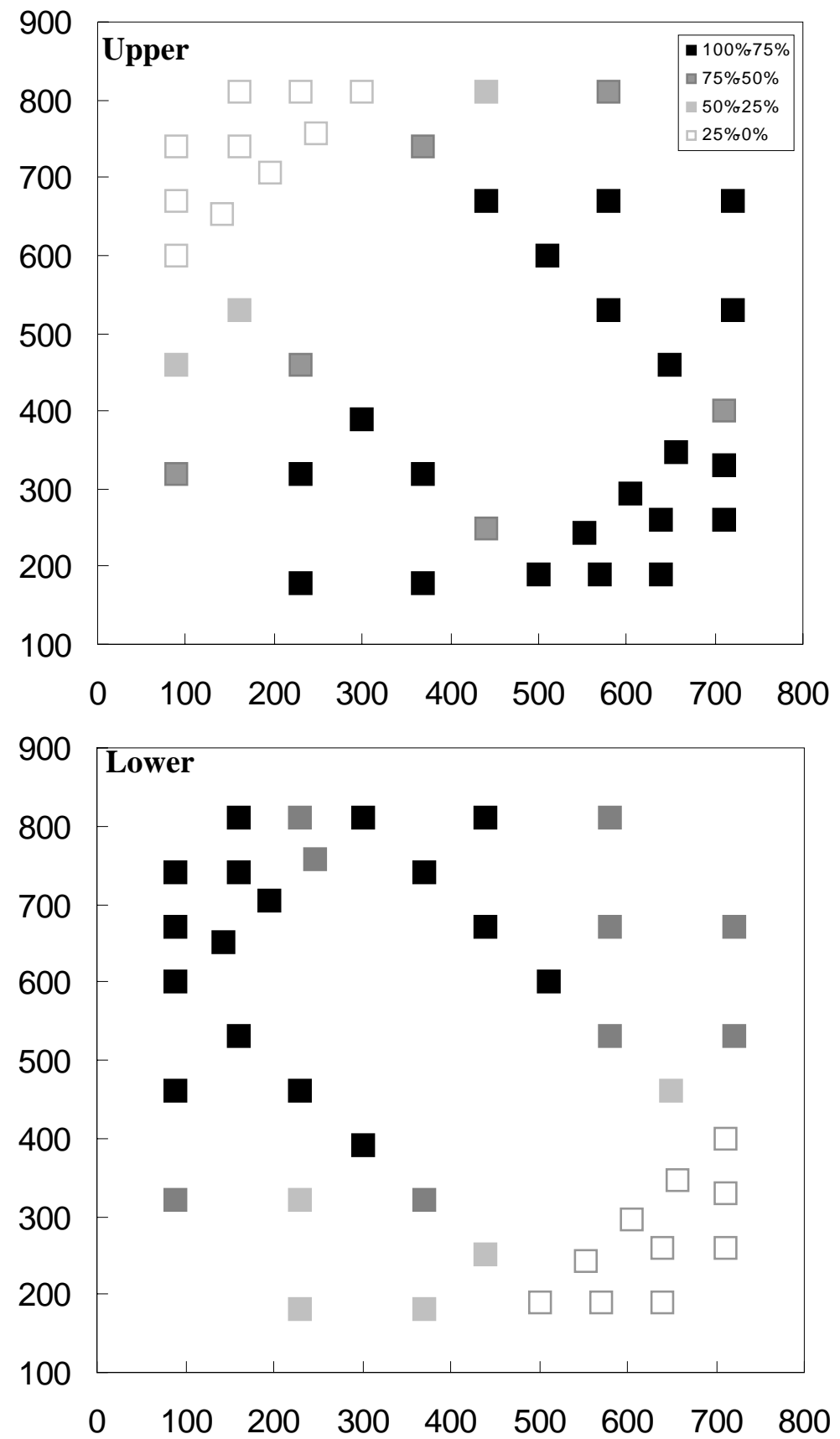

Figure 4.2 The probability of category A responses made by participants in the knowledge partitioning (KP) subgroup for all transfer items in Experiment 1. The darker the fill of a point, the more likely the item was classified as belonging to category A. The upper and lower panels shows the results in the upper and lower context, respectively. 

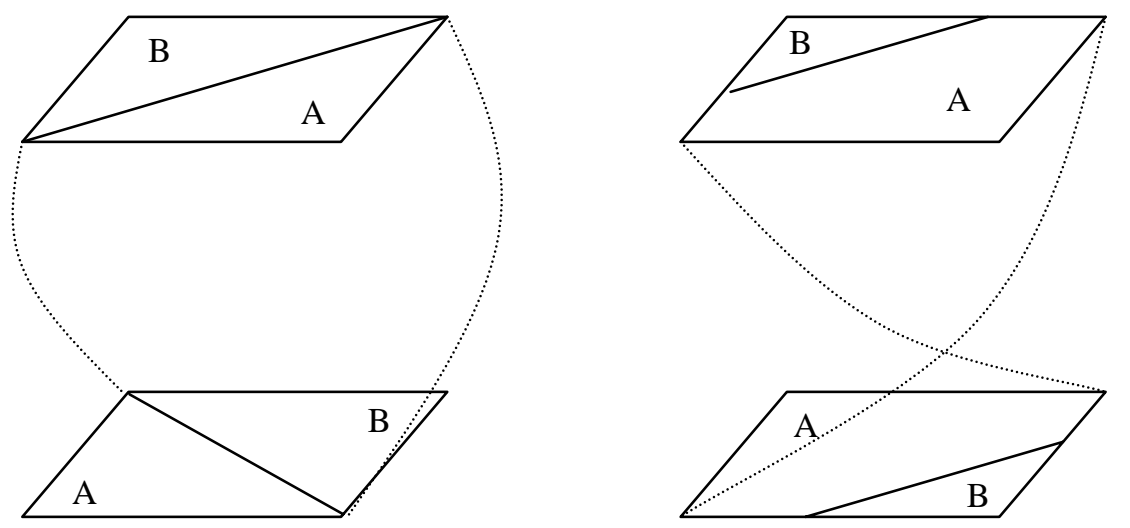

Figure 4.3 Non-linear category structure formed by rotating the linear decision plane. 

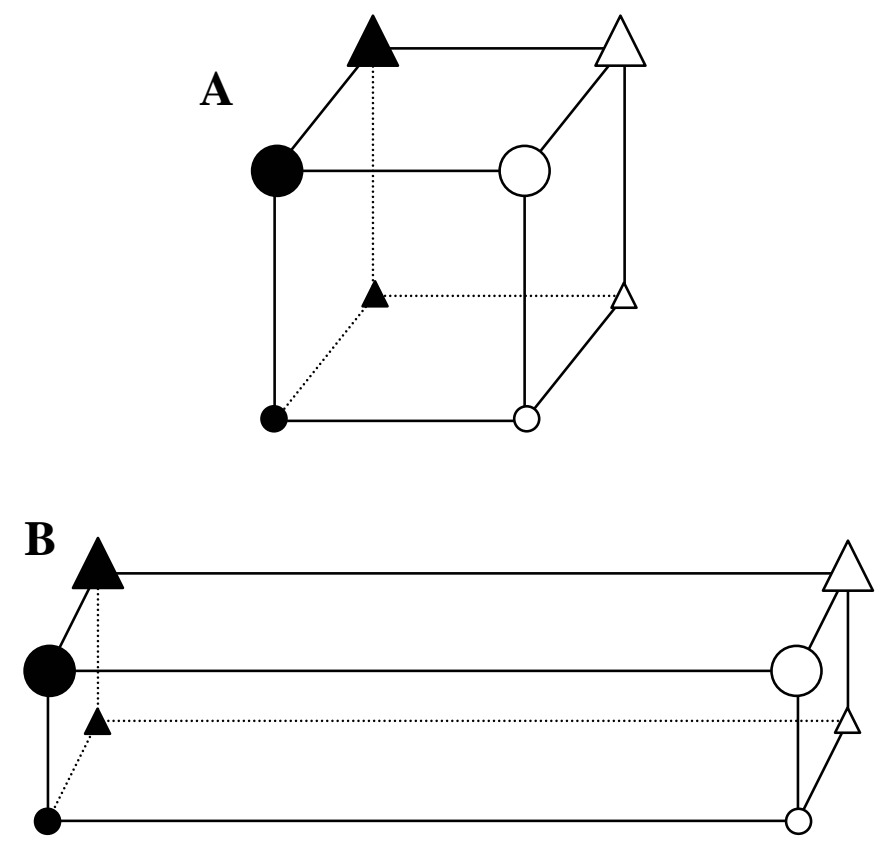

Figure 5.1 Schematic illustration of the attention-optimization hypothesis. 


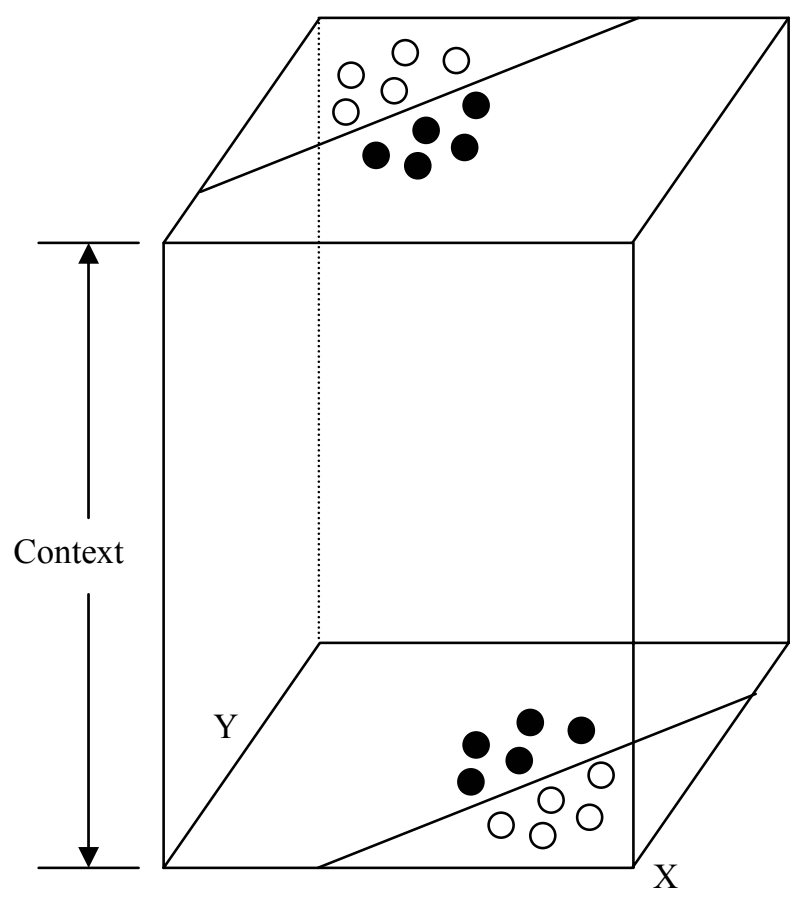

Figure 5.2 Stretching the context dimension results in a 3-D category structure. 


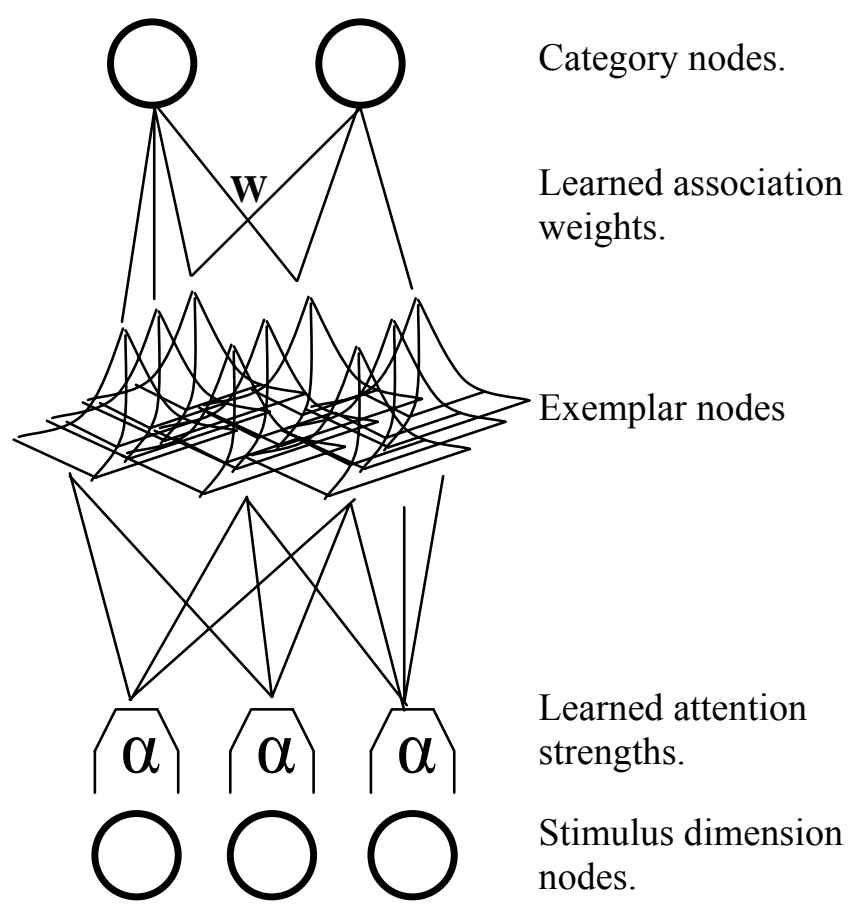

Figure 5.3 The architecture of ALCOVE (attention learning covering map). 

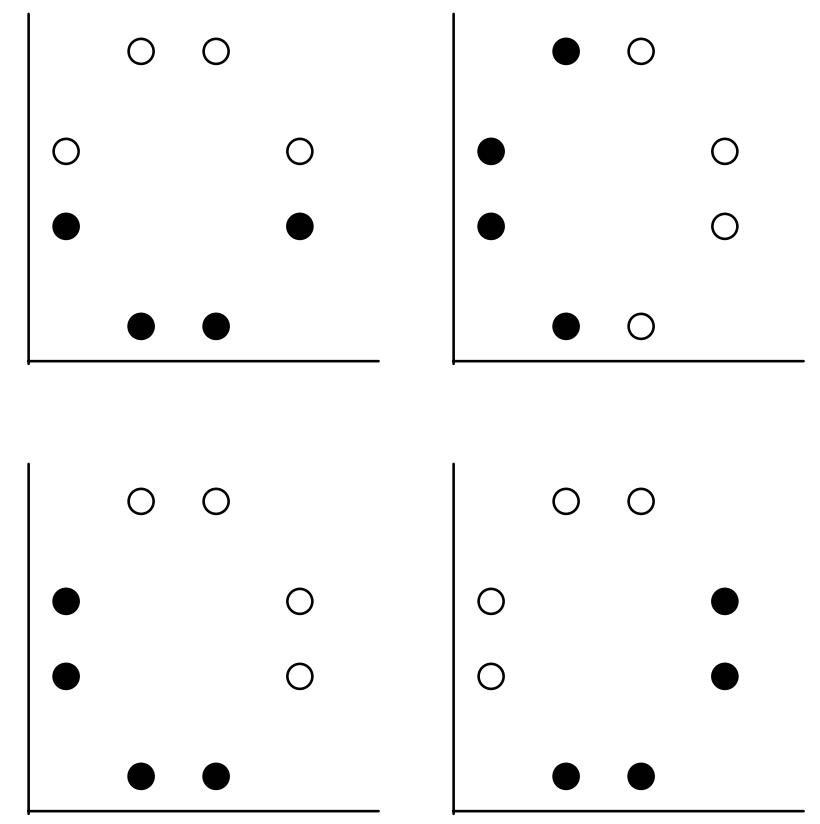

Figure 5.4 Top panel: Division of the structure using horizontal or vertical boundary (filtration task). Bottom panel: Division of the structure using diagonal boundary (condensation task). 


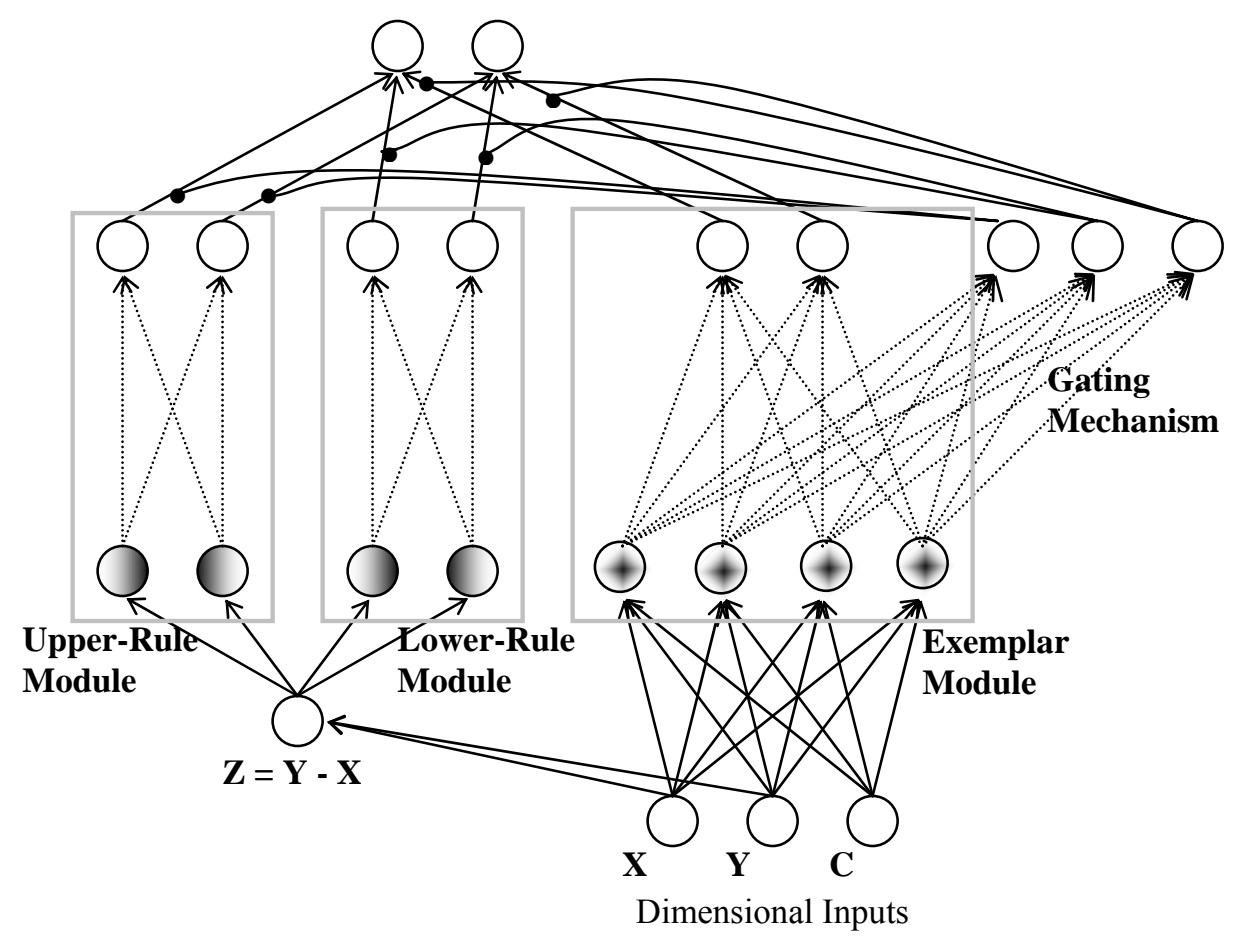

Figure 5.5 The architecture of ATRIUM used to learn a category structure involving two one-dimensional rules. In modeling the data from Experiments 1 and 2, the upper- and lowerrule module was denoted as the left- and right-rule module. 

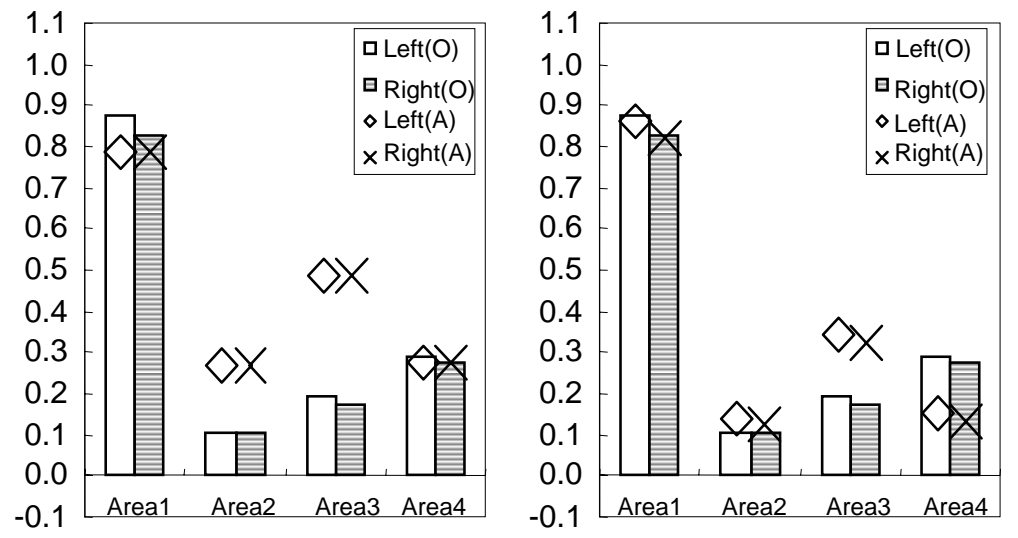

Figure 6.1 The predicted transfer responses of ALCOVE when modeling the randomizedcontext condition in Experiment 1. The left panel shows the predictions when fit to the training data whereas the right panel shows the predictions when fit to the transfer data. The observed category A response is presented by the bars with shading (indicating the context) while ALCOVE's predictions are represented by the symbols (the diamond denotes the upper context and the cross denotes the lower context). 

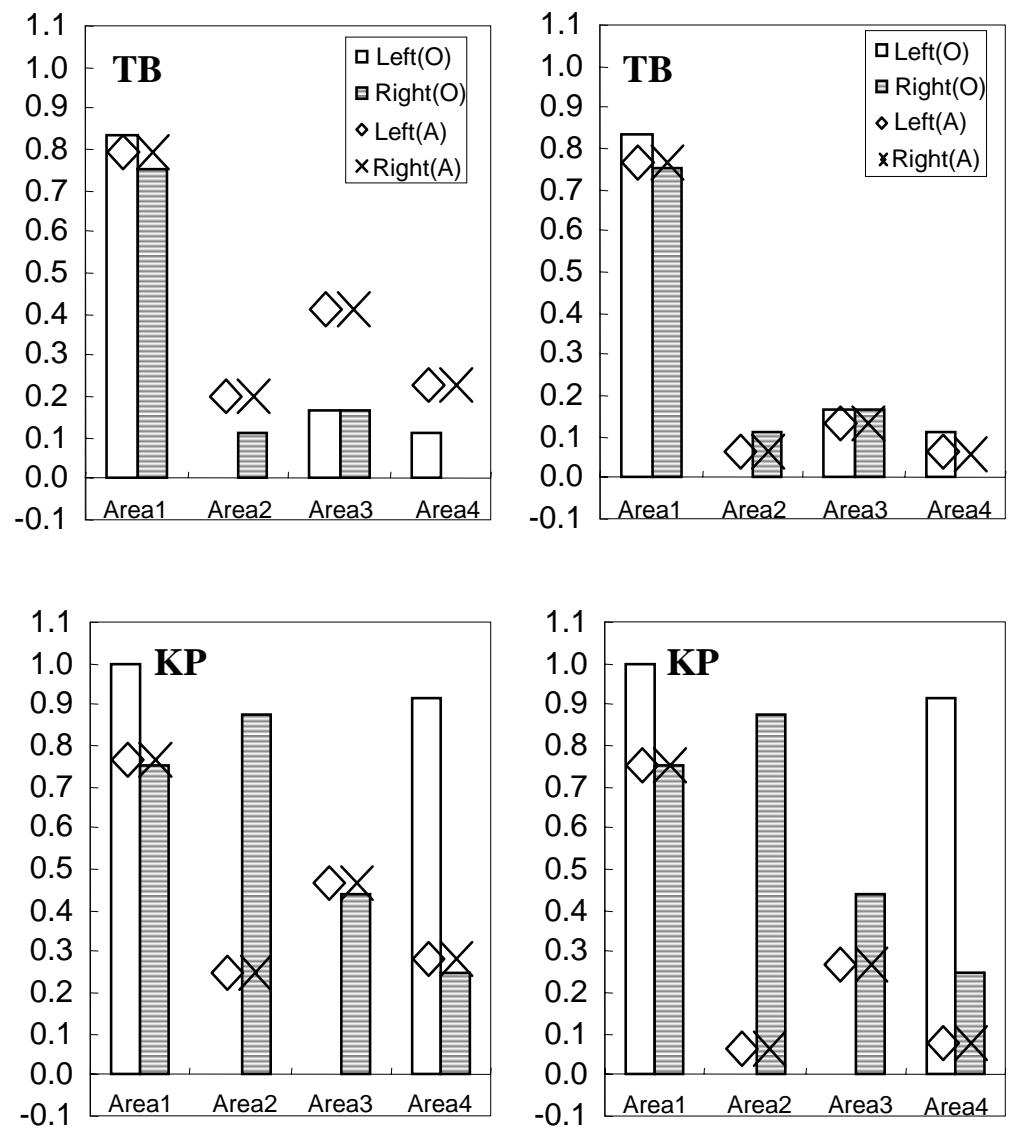

Figure 6.2 The predicted transfer responses of ALCOVE when modeling the systematiccontext condition in Experiment 2. Observed data (vertical bars) for two groups of participants and predictions of ALCOVE (symbols) for the systematic-context condition in Experiment 1. The left panels show predicted transfer performance when ALCOVE was fit to the training data, whereas the right column shows predictions when fit to the transfer data. TB refers to participants who learned the true boundary; KP refers to participants who partitioned their knowledge. Initial attention strengths were .33 for each dimension for all fits. 

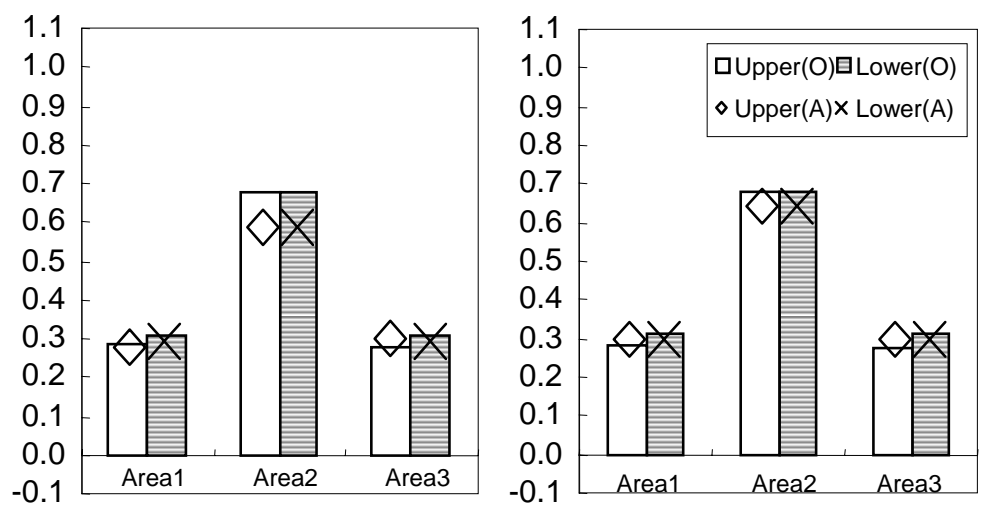

Figure 6.3 Observed data (vertical bars) and predictions of ALCOVE (symbols) for the randomized-context condition in Experiment 5. The left panel shows the predicted transfer performance when the model was fit to the training data, whereas the right panel shows predictions when ALCOVE was fit to the transfer data. 

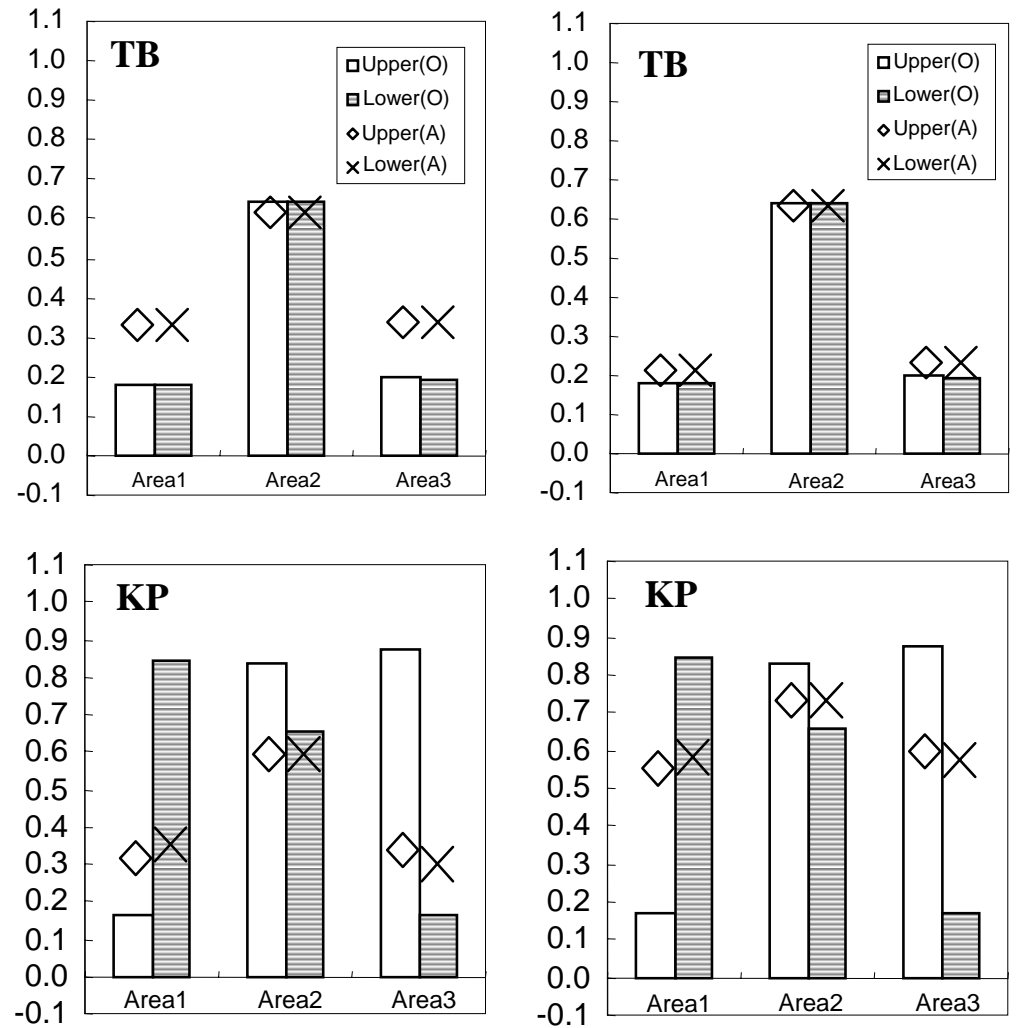

Figure 6.4 Observed data (vertical bars) for two groups of participants and predictions of ALCOVE (symbols) for the systematic-context condition in Experiment 1. The left panels show predicted transfer performance when ALCOVE was fit to the training data, whereas the right column shows predictions when fit to the transfer data. TB refers to participants who learned the true boundary; KP refers to participants who partitioned their knowledge. Initial attention strengths were .33 for each dimension for all fits. 

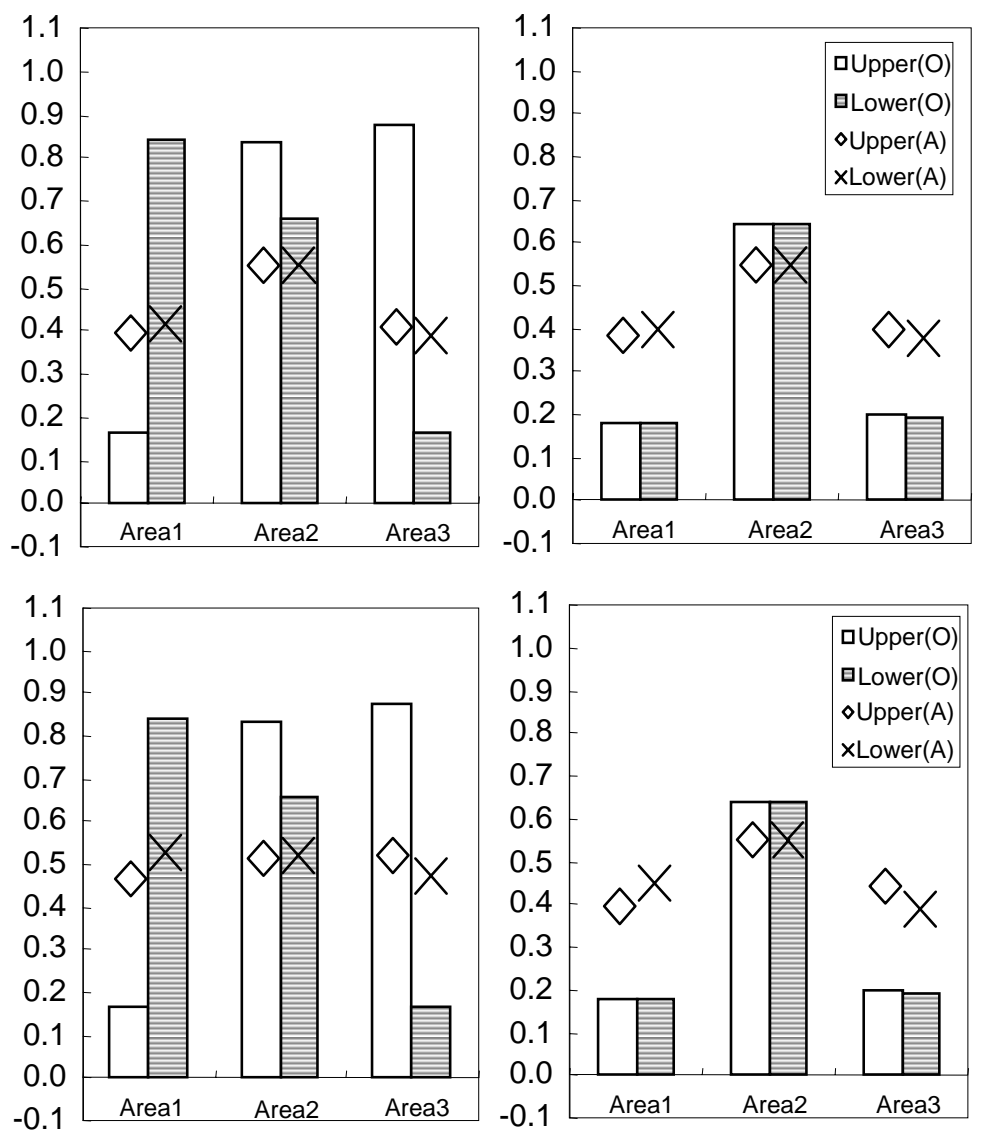

Figure 6.5 The predicted transfer performance of ALCOVE (represented by diamonds and crosses for upper and lower context, respectively) when initial attention strength to context was randomly varied across 100 replications with all parameters kept constant. The left and right columns of panels show predictions for the top and bottom 30\%, respectively, of the distribution of initial attention to context. For the top row of panels, initial attention to context was sampled from a normal distribution with a mean of .15 and standard deviation of .07, whereas for the bottom panels the distribution had a mean of .42 and standard deviation of .15. Observed responses (all from Experiment 5) are represented, respectively, by white and black bars for the upper and lower context. Observed results in the left panel represent the KP group and those in the right panel the TB group. 

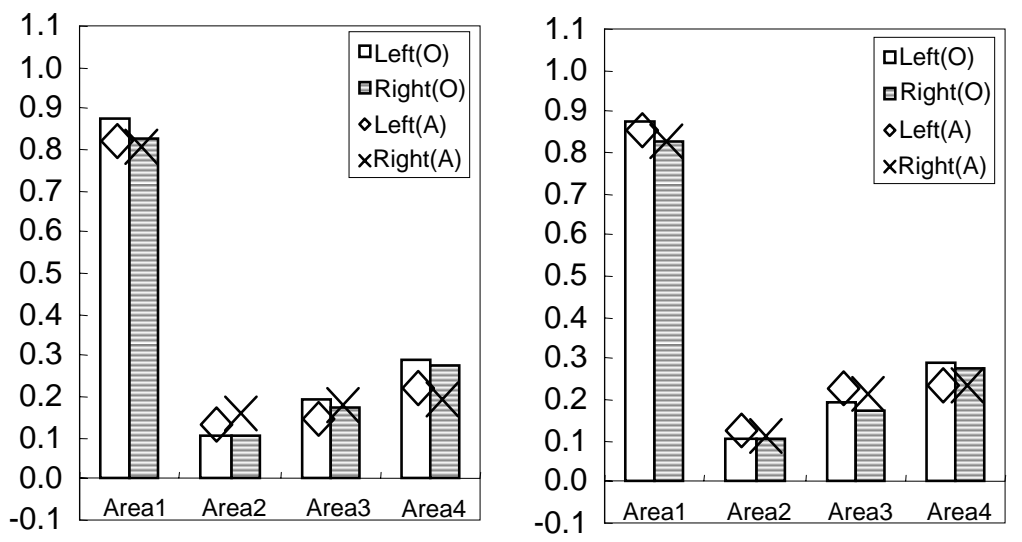

Figure 6.6 Observed data (vertical bars) and predictions of ATRIUM (symbols) for the randomized-context condition in Experiment 1. The left panel shows the predicted transfer performance when the model was fit to the training data, whereas the right panel shows predictions when ATRIUM was fit to the transfer data. 

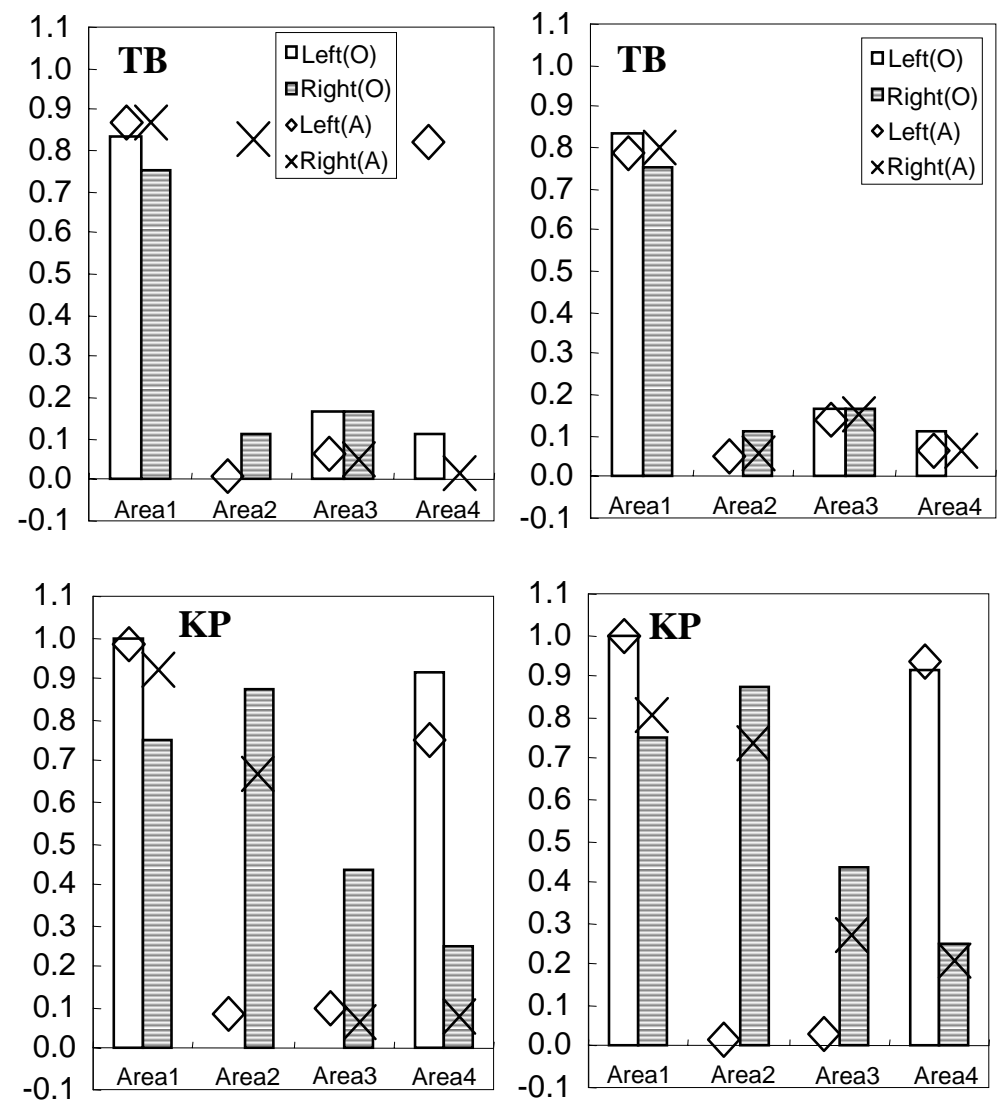

Figure 6.7 Observed data (vertical bars) and predictions of ATRIUM (symbols) for the systematic-context condition in Experiment 2. TB means the true-boundary group; KP means the knowledge partitioning group. The panels in the left column show the predicted transfer performance when the model was fit to the training data, whereas the right panels show predictions when ATRIUM was fit to the transfer data. 


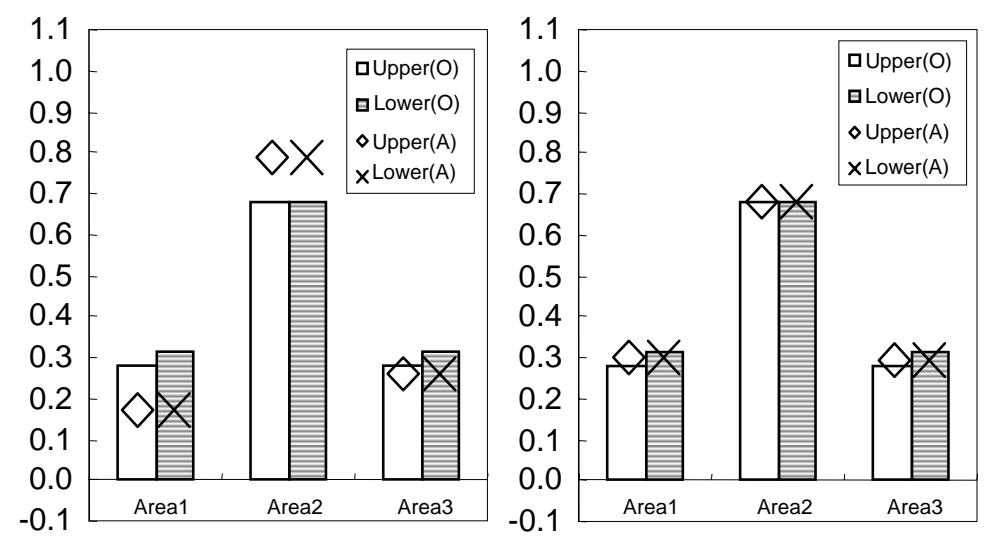

Figure 6.8 Observed data (vertical bars) and predictions of ATRIUM (symbols) for the randomized-context condition in Experiment 5. The left panel shows the predicted transfer performance when the model was fit to the training data, whereas the right panel shows predictions when ATRIUM was fit to the transfer data. 

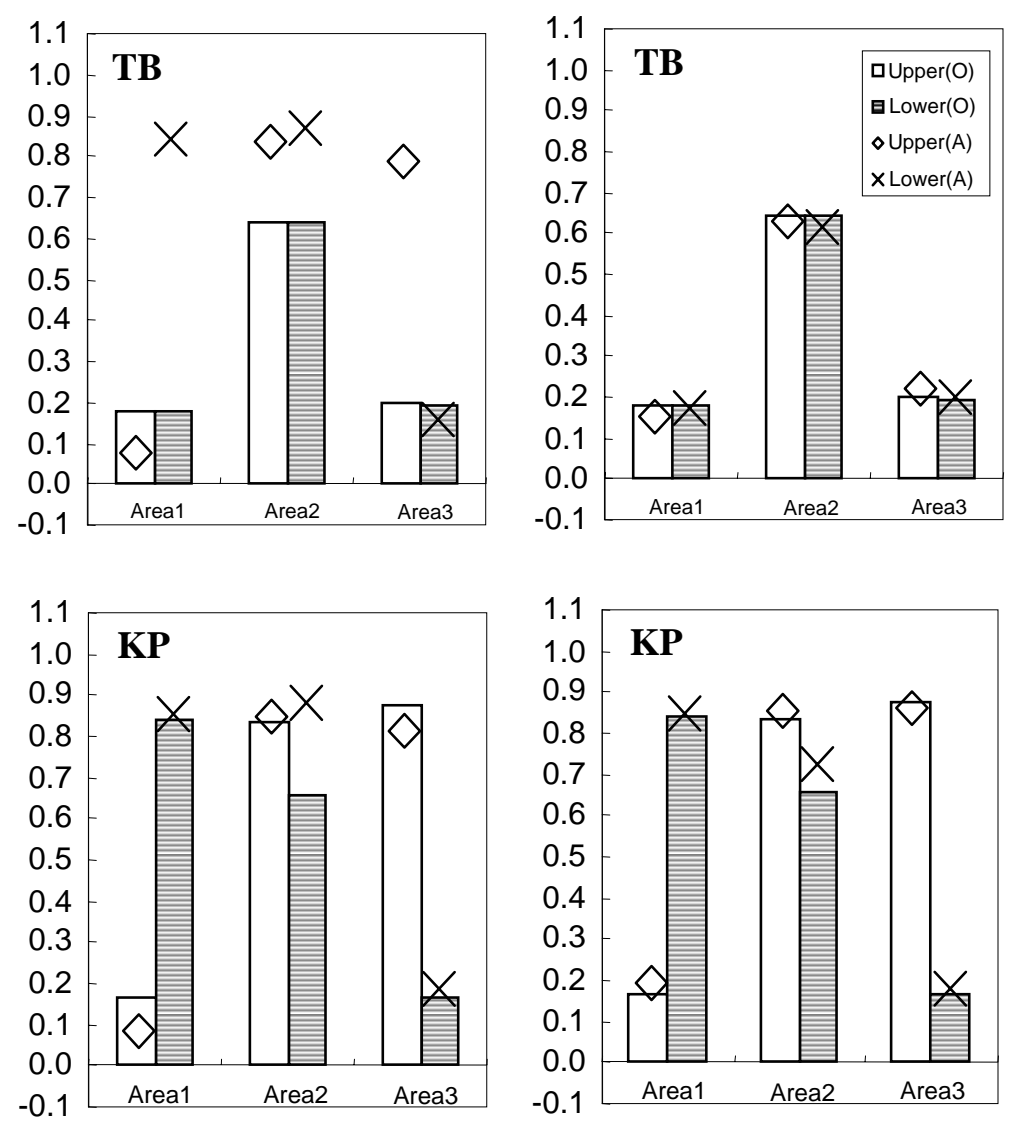

Figure 6.9 Observed data (vertical bars) for two groups of participants and predictions of ATRIUM (symbols) for the systematic-context condition in Experiment 5. The left panels show predicted transfer performance when ATRIUM was fit to the training data, whereas the right column shows predictions when ATRIUM was fit to the transfer data. TB refers to participants who learned the true boundary; KP refers to participants who partitioned their knowledge. 

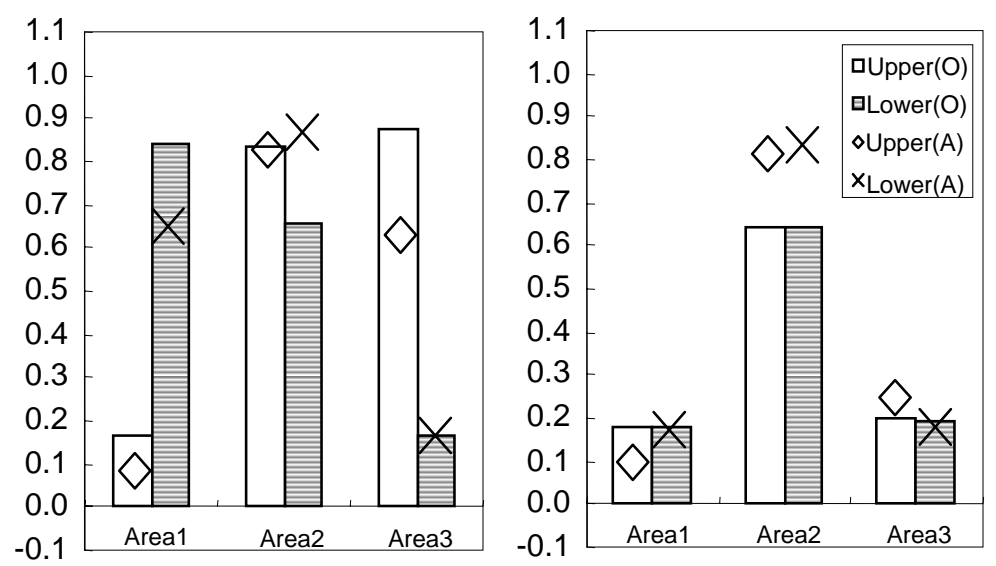

Figure 6.10 The predicted transfer performance of ATRIUM (represented by diamonds and crosses for upper and lower context, respectively) when initial attention strength to context randomly varied across 100 replications with other parameters kept constant. The left and right panels show predictions for the top and bottom 30\%, respectively, of the distribution of initial attention to context. Observed responses (all from Experiment 1) are represented, respectively, by white and black bars for the upper and lower context. Observed results in the left panel represent the KP group and those in the right panel the TB group. KP refers to the knowledge partitioning group, whereas TB refers to the true-boundary group. 

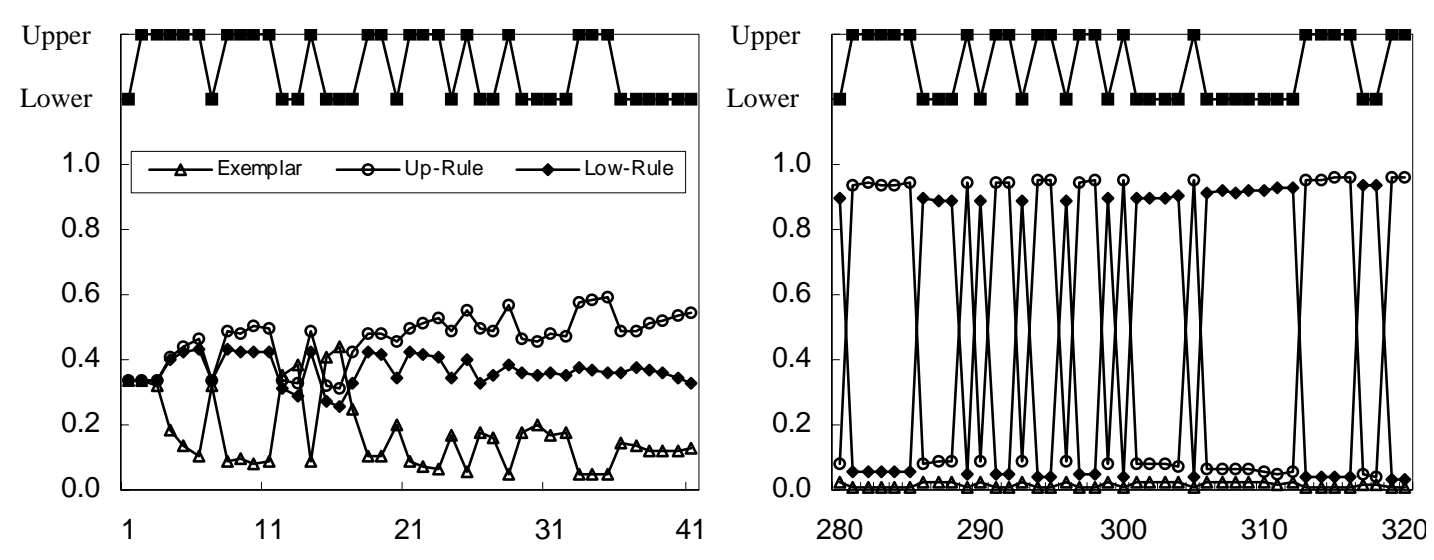

Figure 6.11 The gains assigned to modules on each training trial when fit to the KP group in Experiment 5. The abscissa shows the serial number of each learning trial. The left panel presents for the first learning block and the right panel presents for the final learning block. The chosen probability of the exemplar module, the upper rule module and the lower rule module is depicted by the symbol of white triangle, white circle and black diamond. The top curve denotes which context (upper or lower) in which the trial was presented. 

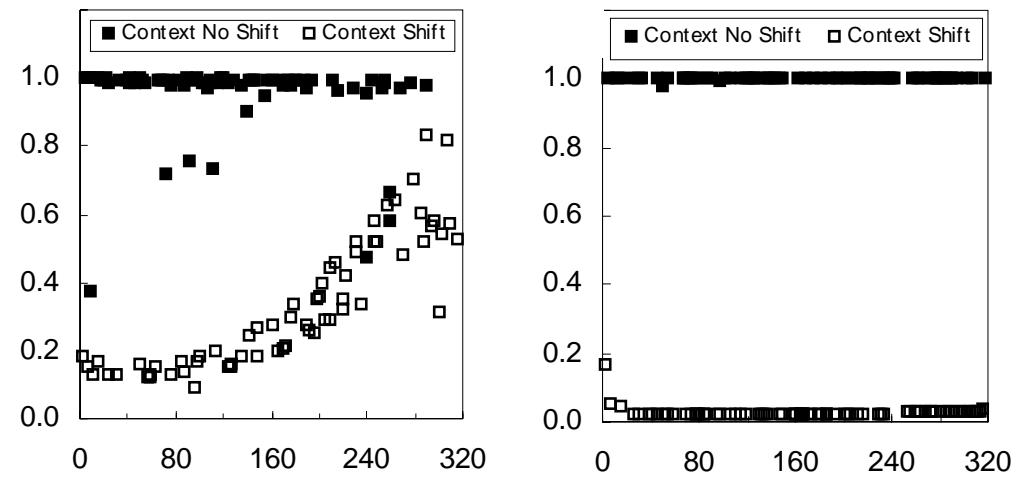

Figure 6.12 The correlation between successive WED vectors in exemplar module. The abscissa denotes the learning trial number and the ordinate denotes the cosine value. KP refers to the knowledge partitioning group, whereas TB refers to the true-boundary group. The left panel shows for the TB group whereas the right on shows for the KP group. The black square denotes no context shift between two successive trials. On the contrary, the white square denotes the context shifts between two successive trials. 

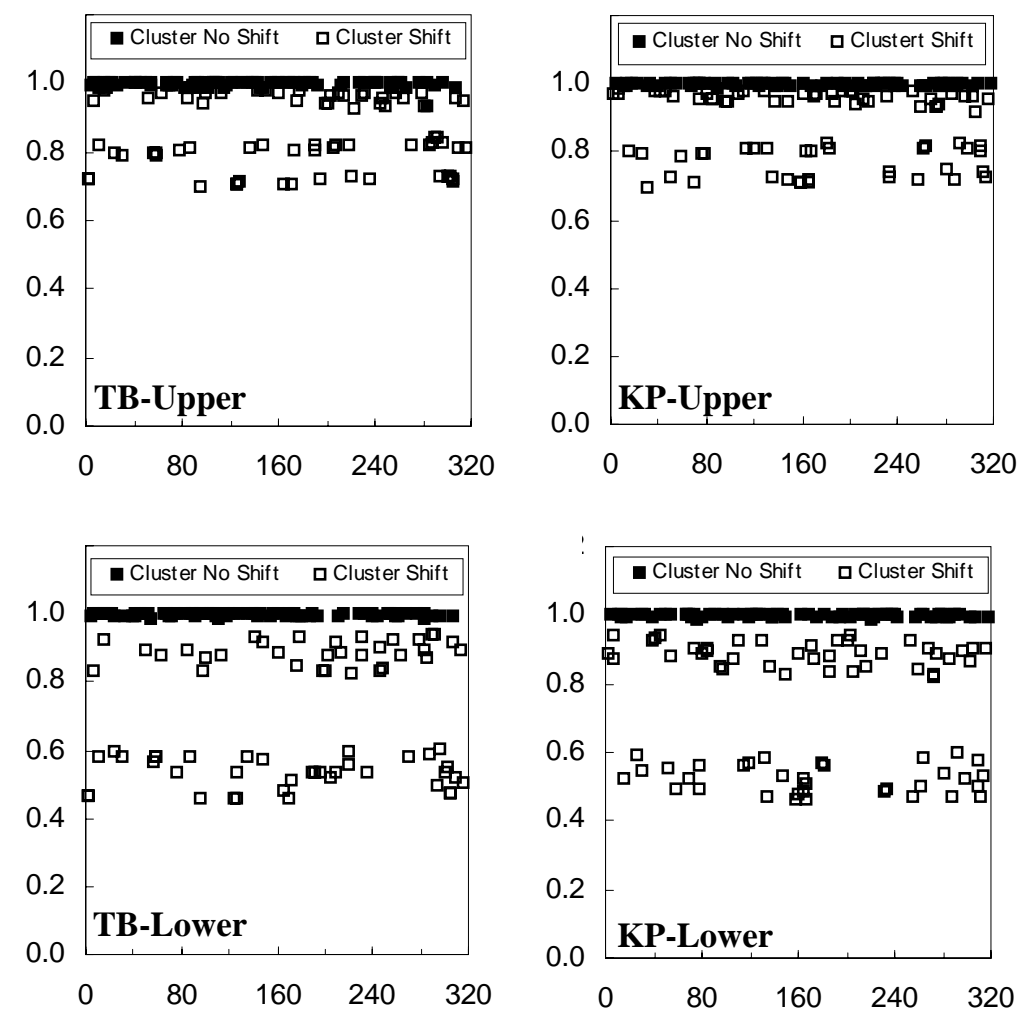

Figure 6.13 The correlation between WED vectors in rule modules. The upper panels depict the upper rule module whereas the lower panels depict for the lower rule module. TB refers to the true-boundary group and KP refers to the knowledge partitioning group. Black square denotes the successive training trials that originate from the same cluster in the $\mathrm{X}-\mathrm{Y}$ space, whereas, white squares denote the successive training trials were chosen from different clusters in the $\mathrm{X}-\mathrm{Y}$ space. 

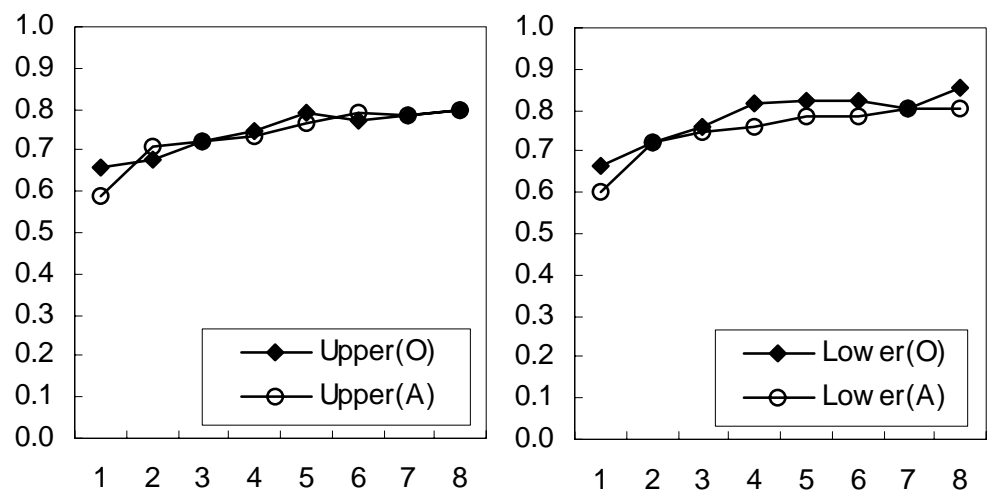

Figure 6.14 The modeling performance of each rule module when fit to the training data from each single-context condition in Experiment 6. The solid diamonds represent the observed proportion correct, whereas the open circles represent the predicted proportion correct. 

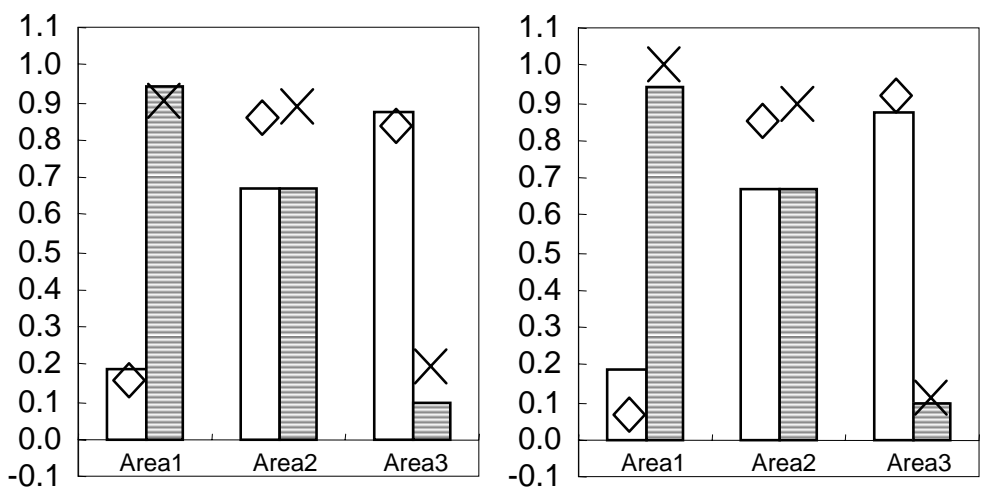

Figure 6.15 Different predictions of ATRIUM in the systematic-context condition when the rule module gains were allowed to vary. 


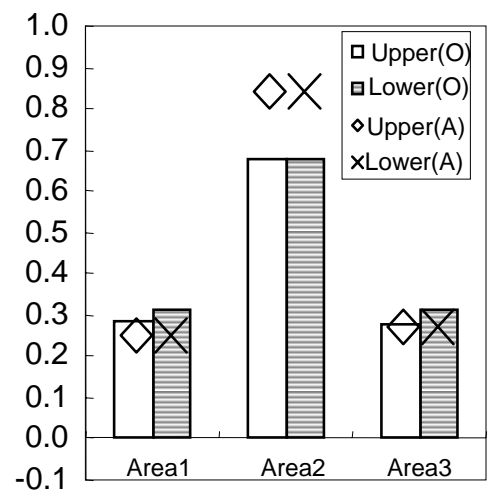

Figure 6.16 ATRIUM's transfer prediction in the randomized-context condition with the rule-module gains allowed to vary. 